\title{
1.11 Primary Radiation Damage Formation
}

\section{R. E. Stoller}

Oak Ridge National Laboratory, Oak Ridge, TN, USA

(c) 2012 Elsevier Ltd. All rights reserved.

\begin{tabular}{|c|c|c|}
\hline 1.11 .1 & Introduction & 293 \\
\hline 1.11 .2 & Description of Displacement Cascades & 294 \\
\hline 1.11.3 & Computational Approach to Simulating Displacement Cascades & 297 \\
\hline 1.11 .4 & Results of MD Cascade Simulations in Iron & 300 \\
\hline 1.11.4.1 & Cascade Evolution and Structure & 303 \\
\hline 1.11.4.2 & Stable Defect Formation & 305 \\
\hline 1.11.4.3 & In-cascade Clustering of Point Defects & 308 \\
\hline 1.11.4.3.1 & Interstitial clustering & 308 \\
\hline 1.11.4.3.2 & Vacancy clustering & 312 \\
\hline 1.11.4.4 & Secondary Factors Influencing Cascade Damage Formation & 315 \\
\hline 1.11.4.4.1 & Influence of preexisting defects & 316 \\
\hline 1.11.4.4.2 & Influence of free surfaces & 318 \\
\hline 1.11.4.4.3 & Influence of grain boundaries & 319 \\
\hline 1.11 .5 & Comparison of Cascade Damage in Other Metals & 323 \\
\hline 1.11.5.1 & Defect Production in Pure Metals & 324 \\
\hline 1.11.5.2 & Defect Production in Fe-C & 325 \\
\hline 1.11.5.3 & Defect Production in $\mathrm{Fe}-\mathrm{Cu}$ & 328 \\
\hline 1.11.5.4 & Defect Production in $\mathrm{Fe}-\mathrm{Cr}$ & 328 \\
\hline 1.11 .6 & Summary and Needs for Further Work & 328 \\
\hline References & & 329 \\
\hline
\end{tabular}

$\begin{array}{ll}\text { Abbreviations } \\ \text { BCA } & \text { Binary collision approximation } \\ \text { COM } & \text { Center of mass } \\ \text { D } & \text { Deuterium } \\ \text { MC } & \text { Monte Carlo } \\ \text { MD } & \text { Molecular dynamics } \\ \text { NN } & \text { Nearest neighbor } \\ \text { NRT } & \text { Norgett, Robinson, and Torrens } \\ \text { PKA } & \text { Primary knock-on atom } \\ \text { RCS } & \text { Replacement collision sequences } \\ \text { SIA } & \text { Self-interstitial atom } \\ \text { T } & \text { Tritium } \\ \text { TEM } & \text { Transmission electron microscope }\end{array}$

\subsubsection{Introduction}

Many of the components used in nuclear energy systems are exposed to high-energy neutrons, which are a by-product of the energy-producing nuclear reactions. In the case of current fission reactors, these neutrons are the result of uranium fission, whereas in future fusion reactors employing deuterium (D) and tritium (T) as fuel, the neutrons are the result of DT fusion. Spallation neutron sources, which are used for a variety of material research purposes, generate neutrons as a result of spallation reactions between a high-energy proton beam and a heavy metal target. Neutron exposure can lead to substantial changes in the microstructure of the materials, which are ultimately manifested as observable changes in component dimensions and changes in the material's physical and mechanical properties as well. For example, radiation-induced void swelling can lead to density changes greater than $50 \%$ in some grades of austenitic stainless steels ${ }^{1}$ and changes in the ductile-to-brittle transition temperature greater than $200{ }^{\circ} \mathrm{C}$ have been observed in the low-alloy steels used in the fabrication of reactor pressure vessels. $^{2,3}$ These phenomena, along with irradiation creep and radiation-induced solute segregation are discussed extensively in the literature ${ }^{4}$ and in more detail elsewhere in this comprehensive volume (e.g., see Chapter 1.03, Radiation-Induced Effects on Microstructure; Chapter 1.04, Effect of Radiation 
on Strength and Ductility of Metals and Alloys; and Chapter 1.05, Radiation-Induced Effects on Material Properties of Ceramics (Mechanical and Dimensional)). The objective of this chapter is to describe the process of primary damage production that gives rise to macroscopic changes. This primary radiation damage event, which is referred to as an atomic displacement cascade, was first proposed by Brinkman in 1954. ${ }^{5,6}$ Many aspects of the cascade damage production discussed below were anticipated in Brinkman's conceptual description.

In contrast to the time scale required for radiationinduced mechanical property changes, which is in the range of hours to years, the primary damage event that initiates these changes lasts only about $10^{-11} \mathrm{~s}$. Similarly, the size scale of displacement cascades, each one being on the order of a few cubic nanometers, is many orders of magnitude smaller than the large structural components that they affect. Although interest in displacement cascades was initially limited to the nuclear industry, cascade damage production has become important in the solid state processing practices of the electronics industry also. The cascades of interest to the electronics industry arise from the use of ion beams to fabricate, modify, or analyze materials for electronic devices. Another related application is the modification of surface layers by ion beam implantation to improve wear or corrosion resistance of materials. ${ }^{8}$ The energy and mass of the particle that initiates the cascade provide the principal differences between the nuclear and ion beam applications. Neutrons from nuclear fission and DT fusion have energies up to about $20 \mathrm{MeV}$ and 14.1 MeV, respectively, while the peak neutron energy in spallation neutron sources reaches as high as the energy of the incident proton beam, $\sim 1 \mathrm{GeV}$ in modern sources. ${ }^{9}$ The neutron mass of one atomic mass unit $\left(1\right.$ a.m.u. $\left.\sim 1.66 \times 10^{-27} \mathrm{~kg}\right)$ is much less than that of the mid-atomic weight metals that comprise most structural alloys. In contrast, many ion beam applications involve relatively low-energy ions, a few tens of kiloelectronvolts, and the mass of both the incident particle and the target is typically a few tens of atomic mass unit. The use of somewhat higher energy ion beams as a tool for investigating neutron irradiation effects is discussed in Chapter 1.07, Radiation Damage Using Ion Beams.

This chapter will focus on the cascade energies of relevance to nuclear energy systems and on iron, which is the primary component in most of the alloys employed in these systems. However, the description of the basic physical mechanisms of displacement cascade formation and evolution given below is generally valid for any crystalline metal and for all of the applications mentioned above. Although additional physical processes may come into play to alter the final defect state in ionic or covalent materials due to atomic charge states, ${ }^{10}$ the ballistic processes observed in metals due to displacement cascades are quite similar in these materials. This has been demonstrated in molecular dynamics (MD) simulations in a range of ceramic materials. ${ }^{11-15}$ Finally, synergistic effects due to nuclear transmutation reactions will not be addressed; the most notable of these, helium production by $(n, \alpha)$ reactions, is the topic of Chapter 1.06, The Effects of Helium in Irradiated Structural Alloys.

\subsubsection{Description of Displacement Cascades}

In a crystalline material, a displacement cascade can be visualized as a series of elastic collisions that is initiated when a given atom is struck by a high-energy neutron (or incident ion in the case of ion irradiation). The initial atom, which is called the primary knock-on atom (PKA), will recoil with a given amount of kinetic energy that it dissipates in a sequence of collisions with other atoms. The first of these are termed secondary knock-on atoms and they will in turn lose energy to a third and subsequently higher ordered knock-ons until all of the energy initially imparted to the PKA has been dissipated. Although the physics is slightly different, a similar event has been observed on billiard tables for many years.

Perhaps the most important difference between billiards and atomic displacement cascades is that an atom in a crystalline solid experiences the binding forces that arise from the presence of the other atoms. This binding leads to the formation of the crystalline lattice and the requirement that a certain minimum kinetic energy must be transferred to an atom before it can be displaced from its lattice site. This minimum energy is called the displacement threshold energy $\left(E_{\mathrm{d}}\right)$ and is typically 20 to $40 \mathrm{eV}$ for most metals and alloys used in structural applications. ${ }^{16}$

If an atom receives kinetic energy in excess of $E_{\mathrm{d}}$, it can be transported from its original lattice site and come to rest within the interstices of the lattice. Such an atom constitutes a point defect in the lattice and is called an interstitial or interstitial atom. In the case of an alloy, the interstitial atom may be referred to as a self-interstitial atom (SIA) if the atom is the primary 
alloy component (e.g., iron in steel) to distinguish it from impurity or solute interstitials. The SIA nomenclature is also used for pure metals, although it is somewhat redundant in that case. The complementary point defect is formed if the original lattice site remains vacant; such a site is called a vacancy (see Chapter 1.01, Fundamental Properties of Defects in Metals for a discussion of these defects and their properties). Vacancies and interstitials are created in equal numbers by this process and the name Frenkel pair is used to describe a single, stable interstitial and its related vacancy. Small clusters of both point defect types can also be formed within a displacement cascade.

The kinematics of the displacement cascade can be described as follows, where for simplicity we consider the case of nonrelativistic particle energies with one particle initially in motion with kinetic energy $E_{0}$ and the other at rest. In an elastic collision between two such particles, the maximum energy $\operatorname{transfer}\left(E_{\mathrm{m}}\right)$ from particle (1) to particle (2) is given by

$$
E_{\mathrm{m}}=4 E_{\mathrm{o}} A_{1} A_{2} /\left(A_{1}+A_{2}\right)^{2}
$$

where $A_{1}$ and $A_{2}$ are the atomic masses of the two particles. Two limiting cases are of interest. If particle 1 is a neutron and particle 2 is a relatively heavy element such as iron, $E_{\mathrm{m}} \sim 4 E_{0} / A$. Alternately, if $A_{1}=A_{2}$, any energy up to $E_{0}$ can be transferred. The former case corresponds to the initial collision between a neutron and the PKA, while the latter corresponds to the collisions between lattice atoms of the same mass.

Beginning with the work of Brinkman mentioned above, various models were proposed to compute the total number of atoms displaced by a given PKA as a function of energy. The most widely cited model was that of Kinchin and Pease. ${ }^{17}$ Their model assumed that between a specified threshold energy and an upper energy cut-off, there was a linear relationship between the number of Frenkel pair produced and the PKA energy. Below the threshold, no new displacements would be produced. Above the high-energy cut-off, it was assumed that the additional energy was dissipated in electronic excitation and ionization. Later, Lindhard and coworkers developed a detailed theory for energy partitioning that could be used to compute the fraction of the PKA energy that was dissipated in the nuclear system in elastic collisions and in electronic losses. ${ }^{18}$ This work was used by Norgett, Robinson, and Torrens (NRT) to develop a secondary displacement model that is still used as a standard in the nuclear industry and elsewhere to compute atomic displacement rates. ${ }^{19}$
The NRT model gives the total number of displaced atoms produced by a PKA with kinetic energy $E_{\mathrm{PKA}}$ as

$$
v_{\mathrm{NRT}}=0.8 T_{\mathrm{d}}\left(E_{\mathrm{PKA}}\right) / 2 E_{\mathrm{d}}^{\prime}
$$

where $E_{\mathrm{d}}$ is an average displacement threshold energy. ${ }^{16}$ The determination of an appropriate average displacement threshold energy is somewhat ambiguous because the displacement threshold is strongly dependent on crystallographic direction, and details of the threshold surface vary from one potential to another. An example of the angular dependence is shown in Figure $1,{ }^{20}$ for MD simulations in iron obtained using the Finnis-Sinclair potential. ${ }^{21}$ Moreover, it is not obvious how to obtain a unique definition for the angular average. Nordlund and coworkers ${ }^{22}$ provide a comparison of threshold behavior obtained with 11 different iron potentials and discusses several different possible definitions of the displacement threshold energy. The factor $T_{\mathrm{d}}$ in eqn [2] is called the damage energy and is a function of $E_{\mathrm{PKA}}$. The damage energy is the amount of the initial PKA energy available to cause atomic displacements, with the fraction of the PKA's initial kinetic energy lost to electronic excitation being responsible for the difference between $E_{\mathrm{PKA}}$ and $T_{\mathrm{d}}$. The ratio of $T_{\mathrm{d}}$ to $E_{\mathrm{PKA}}$ for iron is shown in Figure 2 as a function of PKA energy, where the analytical fit to Lindhard's theory described by Norgett and coworkers ${ }^{19}$ has been used to obtain $T_{\mathrm{d}}$.

Note that a significant fraction of the PKA energy is dissipated in electronic processes even for energies

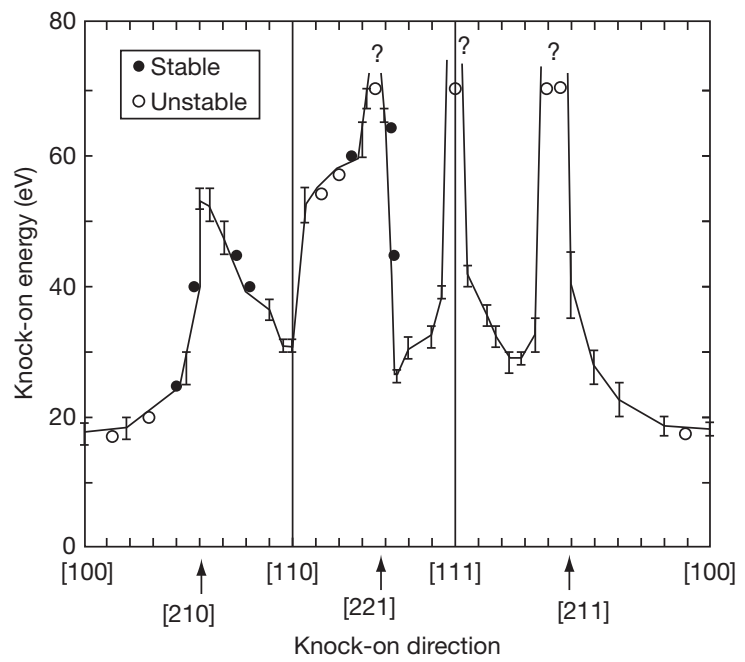

Figure 1 Angular dependence of displacement threshold energy for iron at $0 \mathrm{~K}$. Reproduced from Bacon, D. J.; Calder, A. F.; Harder, J. M.; Wooding, S. J. J. Nucl. Mater. 1993, 205, 52-58. 
as low as a few kiloelectronvolts. The factor of 0.8 in eqn [2] accounts for the effects of realistic (i.e., other than hard sphere) atomic scattering; the value was obtained from an extensive cascade study using the binary collision approximation (BCA). ${ }^{23,24}$

The number of stable displacements (Frenkel pair) predicted by both the original Kinchin-Pease model and the NRT model is shown in Figure 3 as a function of the PKA energy. The third curve in the figure will be discussed below in Section 1.11.3. The MD results presented in Section 1.11.4.2 indicate that $v_{\text {NRT }}$ overestimates the total number of

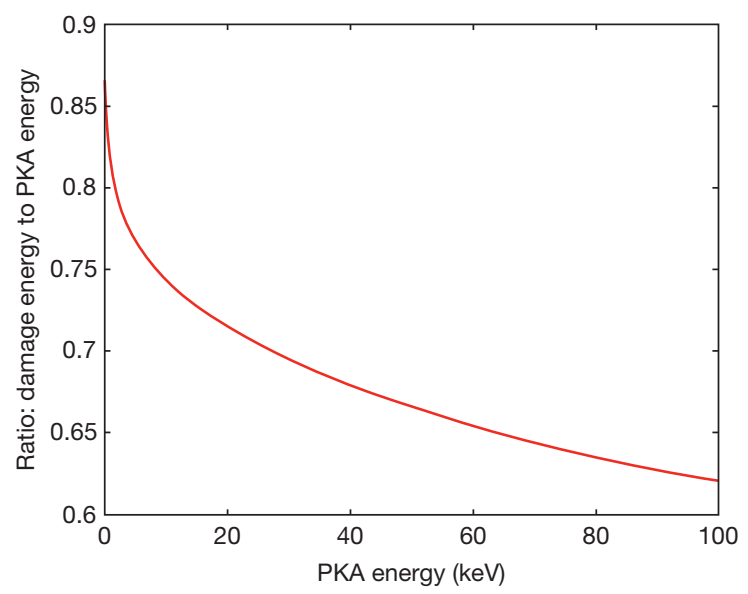

Figure 2 Ratio of damage energy $\left(T_{d}\right)$ to PKA energy $\left(E_{\mathrm{PKA}}\right)$ as a function of PKA energy.
Frenkel pair that remain after the excess kinetic energy in a displacement cascade has been dissipated at about $10 \mathrm{ps}$. Many more defects than this are formed during the collisional phase of the cascade; however, most of these disappear as vacancies and interstitials annihilate one another in spontaneous recombination reactions.

One valuable aspect of the NRT model is that it enabled the use of atomic displacements per atom (dpa) as an exposure parameter, which provides a common basis of comparison for data obtained in different types of irradiation sources, for example, different neutron energy spectra, ion irradiation, or electron irradiation. The neutron energy spectrum can vary significantly from one reactor to another depending on the reactor coolant and/or moderator (water, heavy water, sodium, graphite), which leads to differences in the PKA energy spectrum as will be discussed below. This can confound attempts to correlate irradiation effects data on the basis of parameters such as total neutron fluence or the fluence above some threshold energy, commonly 0.1 or $1.0 \mathrm{MeV}$. More importantly, it is impossible to correlate any given neutron fluence with a charged particle fluence. However, in any of these cases, the PKA energy spectrum and corresponding damage energies can be calculated and the total number of displacements obtained using eqn [2] in an integral calculation. Thus, dpa provides an environmentindependent radiation exposure parameter that in

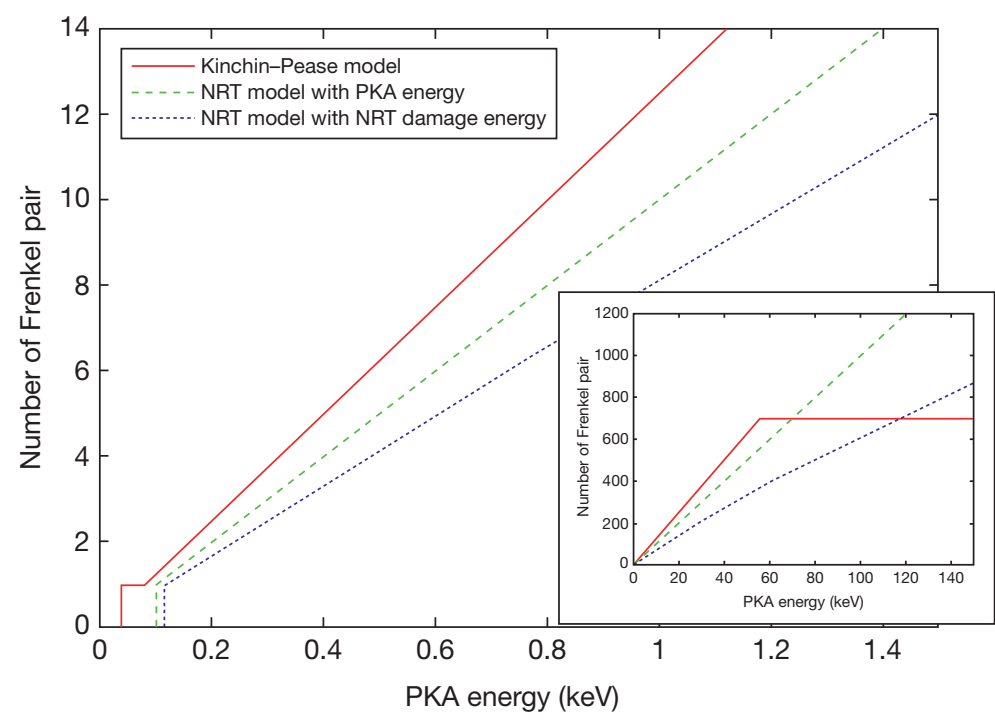

Figure 3 Predicted Frenkel pair production as a function of PKA energy for alternate displacement models (see text for explanation of models). 
many cases can be successfully used as a radiation damage correlation parameter. ${ }^{25}$ As discussed below, aspects of primary damage production other than simply the total number of displacements must be considered in some cases.

\subsubsection{Computational Approach to Simulating Displacement Cascades}

Given the short time scale and small volume associated with atomic displacement cascades, it is not currently possible to directly observe their behavior by any available experimental method. Some of their characteristics have been inferred by experimental techniques that can examine the fine microstructural features that form after low doses of irradiation. The experimental work that provides the best estimate of stable Frenkel pair production involves cryogenic irradiation and subsequent annealing while measuring a parameter such as electrical resistivity. ${ }^{26,27}$ Less direct experimental measurements include small angle neutron scattering, ${ }^{28} \mathrm{X}$-ray scattering, ${ }^{29}$ positron annihilation spectroscopy, ${ }^{30}$ and field ion microscopy. ${ }^{31}$ More broadly, transmission electron microscopy (TEM) has been used to characterize the small point defect clusters such as microvoids, dislocation loops, and stacking fault tetrahedra that are formed as the cascade collapses. ${ }^{32-36}$

The primary tool for investigating radiation damage formation in displacement cascades has been computer simulation using MD, which is a computationally intensive method for modeling atomic systems on the time and length scales appropriate to displacement cascades. The method was pioneered by Vineyard and coworkers at Brookhaven National Laboratory, ${ }^{37}$ and much of the early work on atomistic simulations is collected in a review by Beeler. ${ }^{38}$ Other methods, such as those based on the BCA, ${ }^{20,21}$ have also been used to study displacement cascades. The binary collision models are well suited for very highenergy events, which require that the interatomic potential accurately simulate only close encounters between pairs of atoms. This method requires substantially less computer time than MD but provides less detailed information about lower energy collisions where many-body effects become important. In addition, in-cascade recombination and clustering can only be treated parametrically in the BCA. When the necessary parameters have been calibrated using the results of an appropriate database of MD cascade results, the BCA codes have been shown to reproduce the results of MD simulations reasonably well. ${ }^{39,40}$
A detailed description of the MD method is given in Chapter 1.09, Molecular Dynamics, and will not be repeated here. Briefly, the method relies on obtaining a sufficiently accurate analytical interatomic potential function that describes the energy of the atomic system and the forces on each atom as a function of its position relative to the other atoms in the system. This function must account for both attractive and repulsive forces to obtain the appropriate stable lattice configuration. Specific values for the adjustable coefficients in the function are obtained by ensuring that the interatomic potential leads to reasonable agreement with measured material parameters such as the lattice parameter, lattice cohesive energy, single crystal elastic constants, melting temperature, and point defect formation energies. The process of developing and fitting interatomic potentials is the subject of Chapter 1.10, Interatomic Potential Development. One unique aspect arises when using MD and an empirical potential to investigate radiation damage, viz. the distance of closest approach for highly energetic atoms is much smaller than that obtained in any equilibrium condition. Most potentials are developed to describe equilibrium conditions and must be modified or 'stiffened' to account for these short-range interactions. Chapter 1.10, Interatomic Potential Development, discusses a common approach in which a screened Coulomb potential is joined to the equilibrium potential for this purpose. However, as Malerba points out, ${ }^{41}$ critical aspects of cascade behavior can be sensitive to the details of this joining process.

When this interatomic potential has been derived, the total energy of the system of atoms being simulated can be calculated by summing over all the atoms. The forces on the atoms are obtained from the gradient of the interatomic potential. These forces can be used to calculate the atom's accelerations according to Newton's second law, the familiar $F=m a$ (force $=$ mass $\times$ acceleration), and the equations of motion for the atoms can be solved by numerical integration using a suitably small time step. At the end of the time step, the forces are recalculated for the new atomic positions and this process is repeated as long as necessary to reach the time or state of interest. For energetic PKA, the initial time step may range from $\sim 1$ to $10 \times 10^{-18}$ s, with the maximum time step limited to $\sim 1-10 \times 10^{-15} \mathrm{~s}$ to maintain acceptable numerical accuracy in the integration. As a result, MD cascade simulations are typically not run for times longer than 10-100 ps. With periodic boundary conditions, the size of the simulation cell needs to be 
large enough to prevent the cascade from interacting with periodic images of itself. Higher energy events therefore require a larger number of atoms in the cell. Typical MD cascade energies and the approximate number of atoms required in the simulation are listed in Table 1. With periodic boundaries, it is important that the cell size be large enough to avoid cascade self-interaction. For a given energy, this size depends on the material and, for a given material, on the interatomic potential used. Different interatomic potentials may predict significantly different cascade volumes, even though little variation is eventually found in the number of stable Frenkel pair. ${ }^{42}$ Using a modest number of processors on a modern parallel computer, the clock time required to complete a high-energy simulation with several million atoms is generally less than $48 \mathrm{~h}$. Longer-term evolution of the cascade-produced defect structure can be carried out using Monte Carlo (MC) methods as discussed in Chapter 1.14, Kinetic Monte Carlo Simulations of Irradiation Effects.

The process of conducting a cascade simulation requires two steps. First, a block of atoms of the desired size is thermally equilibrated. This permits the lattice thermal vibrations (phonon waves) to be established for the simulated temperature and typically requires a simulation time of approximately 10 ps. This equilibrated atom block can be saved and used as the starting point for several subsequent cascade simulations. Subsequently, the cascade simulations are initiated by giving one of the atoms a defined amount of kinetic energy, $E_{\mathrm{MD}}$, in a specified direction. Statistical variability can be introduced by either further equilibration of the starting block, choosing a different PKA or PKA direction, or some combination of these. The number of simulations required at any one condition to obtain a good statistical description of defect production is not large. Typically, only about 8-10 simulations are required to obtain a small standard error about the mean number of defects produced; the scatter in defect clustering parameters is larger. This topic will be discussed further below when the results are presented. Most of the cascade simulations discussed below were generated using a [135] PKA direction to minimize directional effects such as channeling and directions with particularly low or high displacement thresholds. The objective has been to determine mean behavior, and investigations of the effect of PKA direction generally indicate that mean values obtained from [135] cascades are representative of the average defect production expected in cascades greater than about $1 \mathrm{keV}^{43} \mathrm{~A}$ stronger influence of PKA direction can be observed at lower energies as discussed in Stoller and coworkers. ${ }^{44,45}$

In the course of the simulation, some procedure must be applied to determine which of the atoms should be characterized as being in a defect state for the purpose of visualization and analysis. One approach is to search the volume of a Wigner-Seitz cell, which is centered on one of the original, perfect lattice sites. An empty cell indicates the presence of a vacancy and a cell containing more than one atom indicates an interstitial-type defect. A more simple geometric criterion has been used to identify defects in most of the results presented below. A sphere with a radius equal to $30 \%$ of the iron lattice parameter is

Table 1 Typical iron atomic displacement cascade parameters

\begin{tabular}{|c|c|c|c|c|c|}
\hline $\begin{array}{l}\text { Neutron energy } \\
\text { (MeV) }\end{array}$ & $\begin{array}{l}\text { Average } P K A \\
\text { energy }(k e V)^{\mathrm{a}}\end{array}$ & $\begin{array}{l}\text { Corresponding } \\
\mathrm{T}_{d}(\mathrm{keV})^{\mathrm{b}} \sim \mathrm{E}_{M D}\end{array}$ & $\begin{array}{l}\text { NRT } \\
\text { displacements }\end{array}$ & $\begin{array}{l}\text { Ratio: } \\
\mathrm{T}_{d} / \mathrm{E}_{P K A}\end{array}$ & $\begin{array}{l}\text { Typical simulation } \\
\text { cell size (atoms) }\end{array}$ \\
\hline 0.00335 & 0.116 & 0.1 & 1 & 0.8634 & 3456 \\
\hline 0.00682 & 0.236 & 0.2 & 2 & 0.8487 & 6750 \\
\hline 0.0175 & 0.605 & 0.5 & 5 & 0.8269 & \\
\hline 0.0358 & 1.24 & 1.0 & 10 & 0.8085 & 54000 \\
\hline 0.0734 & 2.54 & 2.0 & 20 & 0.7881 & \\
\hline 0.191 & 6.6 & 5.0 & 50 & 0.7570 & 128000 \\
\hline 0.397 & 13.7 & 10.0 & 100 & 0.7292 & 250000 \\
\hline 0.832 & 28.8 & 20.0 & 200 & 0.6954 & $\sim 500 \mathrm{k}$ \\
\hline 2.28 & 78.7 & 50.0 & 500 & 0.6354 & $\sim 2.5 \mathrm{M}$ \\
\hline 5.09 & 175.8 & 100.0 & 1000 & 0.5690 & $\sim 5-10 \mathrm{M}$ \\
\hline 12.3 & 425.5 & 200.0 & 2000 & 0.4700 & $\sim 10-20 \mathrm{M}$ \\
\hline $14.1^{c}$ & 487.3 & 220.4 & 2204 & 0.4523 & \\
\hline
\end{tabular}

${ }^{a}$ This is the average iron recoil energy from an elastic collision with a neutron of the specified energy.

'Damage energy calculated using Robinson's approximation to LSS theory. ${ }^{19}$

${ }^{c}$ Relevant to D-T fusion energy production. 
centered on the perfect lattice sites, and a search similar to that just described for the Wigner-Seitz cell is carried out. Any atom that is not within such a sphere is identified as part of an interstitial defect and each empty sphere identifies the location of a vacancy. The diameter of the effective sphere is slightly less than the spacing of the two atoms in a dumbbell interstitial (see below). A comparison of the effective sphere and Wigner-Seitz cell approaches found no significant difference in the number of stable point defects identified at the end of cascade simulation, and the effective sphere method is faster computationally. The drawback to this approach is that the number of defects identified by the algorithm must be corrected to account for the nature of the interstitial defect that is formed. In order to minimize the lattice strain energy, most interstitials are found in the dumbbell configuration; the energy is reduced by distributing the distortion over multiple lattice sites. In this case, the single interstitial appears to be composed of two interstitials separated by a vacancy. In other cases, the interstitial configuration is extended further, as in the case of the crowdion in which an interstitial may be visualized as three displaced atoms and two empty lattice sites. These interstitial configurations are illustrated in Figure 4, which uses the convention adopted throughout this chapter, that is, vacancies are displayed as red spheres and interstitials as green spheres. A simple postprocessing code was used to determine the true number of point defects, which are reported below.

Most MD codes describe only the elastic collisions between atoms; they do not account for energy

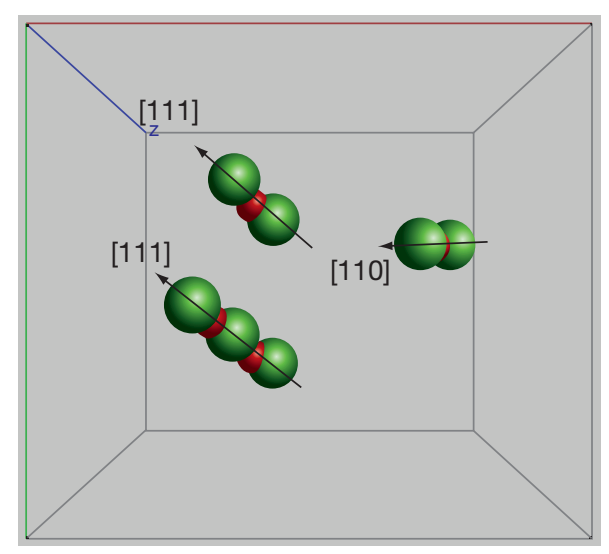

Figure 4 Typical configurations for interstitials created in displacement cascades: [110] and [111] dumbbells and [111] crowdion. loss mechanisms such as electronic excitation and ionization. Thus, the initial kinetic energy, $E_{\mathrm{MD}}$, given to the simulated PKA in MD simulations is more analogous to $T_{\mathrm{d}}$ in eqn [2] than it is to the PKA energy, which is the total kinetic energy of the recoil in an actual collision. Using the values of $E_{\mathrm{MD}}$ in Table 1 as a basis, the corresponding $E_{\mathrm{PKA}}$ and $v_{\mathrm{NRT}}$ for iron, and the ratio of the damage energy to the PKA energy, have been calculated using the procedure described in Norgett and coworkers. ${ }^{19}$ and the recommended $40 \mathrm{eV}$ displacement threshold. ${ }^{16}$ These values are also listed in Table 1 , along with the neutron energy that would yield $E_{\mathrm{PKA}}$ as the average recoil energy in iron. This is one-half of the maximum energy given by eqn [1]. As mentioned above, the difference between the MD cascade energy, or damage energy, and the PKA energy increases as the PKA energy increases. Discussions of cascade energy in the literature on MD cascade simulations are not consistent with respect to the use of the term PKA energy. The third curve in Figure 3 shows the calculated number of Frenkel pair predicted by the NRT model if the PKA energy is used in eqn [2] rather than the damage energy. The difference between the two sets of NRT values is substantial and is a measure of the ambiguity associated with being vague in the use of terminology. It is recommended that the MD cascade energy should not be referred to as the PKA energy. For the purpose of comparing MD results to the NRT model, the MD cascade energy should be considered as approximately equal to the damage energy ( $T_{\mathrm{d}}$ in eqn [2]).

In reality, energetic atoms lose energy continuously by a combination of electronic and nuclear reactions, and the typical MD simulation effectively deletes the electronic component at time zero. The effects of continuous energy loss on defect production have been investigated in the past using a damping term to slowly remove kinetic energy. ${ }^{46}$ The related issues of how this extracted energy heats the electron system and the effects of electron-phonon coupling on local temperature have also been examined. ${ }^{47-50}$ More recently, computational and algorithmic advances have enabled these phenomena to be investigated with higher fidelity. ${ }^{51}$ Some of the work just referenced has shown that accounting for the electronic system has a modest quantitative effect on defect formation in displacement cascades. For example, Gao and coworkers found a systematic increase in defect formation as they increased the effective electron-phonon coupling in 2, 5, and $10 \mathrm{keV}$ cascade simulations in iron, ${ }^{50}$ and a similar effect was reported 
by Finnis and coworkers. ${ }^{47}$ However, the primary physical mechanisms of defect formation that are the focus of this chapter can be understood in the absence of these effects.

\subsubsection{Results of MD Cascade Simulations in Iron}

MD simulations have been employed to investigate displacement cascade evolution in a wide range of materials. The literature is sufficiently broad that any list of references will be necessarily incomplete; Malerba, ${ }^{41}$ Stoller, ${ }^{43}$ and others ${ }^{52-70}$ provide only a representative sample. Additional references will be given below as specific topics are discussed. The recent review by Malerba ${ }^{41}$ provides a good summary of the research that has been done on iron. These MD investigations of displacement cascades have established several consistent trends in primary damage formation in a number of materials. These trends include (1) the total number of stable point defects produced follows a power-law dependence on the cascade energy over a broad energy range, (2) the ratio of MD stable displacements divided by the number obtained from the NRT model decreases with energy until subcascade formation becomes prominent, (3) the in-cascade clustering fraction of the surviving defects increases with cascade energy, and (4) the effect of lattice temperature on the MD results is rather weak. Two additional observations have been made regarding in-cascade clustering in iron, although the fidelity of these statements depends on the interatomic potential employed. First, the interstitial clusters have a complex, three-dimensional (3D) morphology, with both sessile and glissile configurations. Mobile interstitial clusters appear to glide with a low activation energy similar to that of the monointerstitial $(\sim 0.1-0.2 \mathrm{eV}) .{ }^{71}$ Second, the fraction of the vacancies contained in clusters is much lower than the interstitial clustering fraction. Each of these points will be discussed further below.

The influence of the interatomic potential on cascade damage production has been investigated by several researchers. ${ }^{42,72-74}$ Such comparisons generally show only minor quantitative differences between results obtained with interatomic potentials of the same general type, although the differences in clustering behavior are more significant with some potentials. Variants of embedded atom or Finnis-Sinclair type potential functions (see Chapter 1.10, Interatomic Potential Development) have most often been used.
However, more substantial differences are sometimes observed that are difficult to correlate with any known aspect of the potentials. The analysis recently reported by Malerba ${ }^{41}$ is one example. In this case, it appears that the formation of replacement collision sequences (RCS) (discussed in Section 1.11.4.1) was very sensitive to the range over which the equilibrium part of the potential was joined to the more repulsive pair potential that controls short-range interactions. This changed the effective cascade energy density and thereby the number of stable defects produced.

Therefore, in order to provide a self-consistent database for illustrating cascade damage production over a range of temperatures and energies and to provide examples of secondary variables that can influence this production, the results presented in this chapter will focus on MD simulations in iron using a single interatomic potential. ${ }^{43,53,54,64-68}$ This potential was originally developed by Finnis and Sinclair $^{21}$ and later modified for cascade simulations by Calder and Bacon. ${ }^{58}$ The calculations were carried out using a modified version of the MOLDY code written by Finnis. ${ }^{75}$ The computing time with this code is almost linearly proportional to the number of atoms in the simulation. Simulations were carried out using periodic, Parrinello-Rahman boundary conditions at constant pressure. ${ }^{76}$ As no thermostat was applied to the boundaries, the average temperature of the simulation cell was increased as the kinetic energy of the PKA was dissipated. The impact of this heating appears to be modest based on the observed effects of irradiation temperature discussed below, and on the results observed in the work of Gao and coworkers. ${ }^{77} \mathrm{~A}$ brief comparison of the iron cascade results with those obtained in other metals will be presented in Section 1.11.5.

The primary variables studied in these cascade simulations is the cascade energy, $E_{\mathrm{MD}}$, and the irradiation temperature. The database of iron cascades includes cascade energies from near the displacement threshold $(\sim 100 \mathrm{eV})$ to a $200 \mathrm{keV}$, and temperatures in the range of 100-900 K. In all cases, the evolution of the cascade has been followed to completion and the final defect state determined. Typically this is reached after a few picoseconds for the low-energy cascades and up to $\sim 15$ ps for the highest energy cascades. Because of the variability in final defect production for similar initial conditions, several simulations were conducted at each energy to produce statistically meaningful average values. The parameters of most interest from these studies are the number of surviving point defects, the fraction of 
these defects that are found in clusters, and the size distribution of the point defect clusters. The total number of point defects is a direct measure of the residual radiation damage and the potential for longrange mass transport and microstructural evolution. In-cascade defect clustering is important because it can promote microstructural evolution by eliminating the cluster nucleation phase.

The parameters used in the following discussion to describe results of MD cascade simulations are the total number of surviving point defects and the fraction of the surviving defects contained in clusters. The number of surviving defects will be expressed as a fraction of the NRT displacements listed in Table 1, whereas the number of defects in clusters will be expressed as either a fraction of the NRT displacements or a fraction of the total surviving MD defects. Alternate criteria were used to define a point defect cluster in this study. In the case of interstitial clusters, it was usually determined by direct visualization of the defect structures. The coordinated movement of interstitials in a given cluster can be clearly observed. Interstitials bound in a given cluster were typically within a second nearest-neighbor (NN) distance, although some were bound at third NN. The situation for vacancy clusters will be discussed further below, but vacancy clustering was assessed using first, second, third, and fourth NN distances as the criteria. The vacancy clusters observed in iron tend to not exhibit a compact structure according to these definitions. In order to analyze the statistical variation in the primary damage parameters, the mean value $(M)$, the standard deviation about the mean $(\sigma)$, and the standard error of the mean $(\varepsilon)$ have been calculated for each set of cascades conducted at a given energy and temperature. The standard error of the mean is calculated as $\varepsilon=\sigma / n^{0.5}$, where $n$ is the number of cascade simulations completed. ${ }^{78}$ The standard error of the mean provides a measure of how well the sample mean represents the actual mean. For example, a $90 \%$ confidence limit on the mean is obtained from $1.86 \varepsilon$ for a sample size of nine. ${ }^{79}$ These statistical quantities are summarized in Table 2 for a representative subset of the iron cascade database.

Table 2 Statistical analysis of primary damage parameters derived from MD cascade simulations

\begin{tabular}{|c|c|c|c|c|c|c|c|}
\hline \multirow[t]{2}{*}{ Energy (keV) } & \multirow[t]{2}{*}{ Temperature (K) } & \multirow[t]{2}{*}{ Number of cascades } & \multicolumn{2}{|c|}{$\begin{array}{l}\text { Surviving MD } \\
\text { displacements } \\
\text { (mean / standard } \\
\text { deviation / standard } \\
\text { error) }\end{array}$} & \multicolumn{3}{|c|}{$\begin{array}{l}\text { Clustered interstitials (mean / } \\
\text { standard deviation / standard error) }\end{array}$} \\
\hline & & & Number & per NRT & Number & per NRT & $\begin{array}{l}\text { per MD } \\
\text { surviving } \\
\text { defects }\end{array}$ \\
\hline & & & 3.94 & 0.790 & 1.25 & 0.250 & 0.310 \\
\hline 0.5 & 100 & 16 & $\begin{array}{l}0.680 \\
0.170\end{array}$ & $\begin{array}{l}0.136 \\
0.0340\end{array}$ & $\begin{array}{l}1.39 \\
0.348\end{array}$ & $\begin{array}{l}0.278 \\
0.0695\end{array}$ & $\begin{array}{l}0.329 \\
0.0822\end{array}$ \\
\hline 1 & 100 & 12 & $\begin{array}{l}6.08 \\
1.38 \\
0.398\end{array}$ & $\begin{array}{l}0.608 \\
0.138 \\
0.0398\end{array}$ & $\begin{array}{l}2.25 \\
1.66 \\
0.479\end{array}$ & $\begin{array}{l}0.225 \\
0.166 \\
0.0479\end{array}$ & $\begin{array}{l}0.341 \\
0.248 \\
0.0715\end{array}$ \\
\hline 1 & 600 & 12 & $\begin{array}{l}5.25 \\
2.01 \\
0.579\end{array}$ & $\begin{array}{c}0.525 \\
0.201 \\
.0579\end{array}$ & $\begin{array}{l}1.92 \\
2.02 \\
0.583\end{array}$ & $\begin{array}{l}0.192 \\
0.202 \\
0.0583\end{array}$ & $\begin{array}{l}0.307 \\
0.327 \\
0.0944\end{array}$ \\
\hline 1 & 900 & 12 & $\begin{array}{l}4.33 \\
1.07 \\
0.310\end{array}$ & $\begin{array}{l}0.433 \\
0.107 \\
0.031\end{array}$ & $\begin{array}{l}1.00 \\
1.28 \\
0.369\end{array}$ & $\begin{array}{l}0.100 \\
0.128 \\
0.0369\end{array}$ & $\begin{array}{l}0.221 \\
0.287 \\
0.0829\end{array}$ \\
\hline 2 & 100 & 10 & $\begin{array}{l}10.1 \\
2.64 \\
0.836\end{array}$ & $\begin{array}{l}0.505 \\
0.132 \\
0.0418\end{array}$ & $\begin{array}{l}4.60 \\
2.80 \\
0.884\end{array}$ & $\begin{array}{l}0.230 \\
0.140 \\
0.0442\end{array}$ & $\begin{array}{l}0.432 \\
0.0214 \\
0.00678\end{array}$ \\
\hline 5 & 100 & 9 & $\begin{array}{l}22.0 \\
2.12 \\
0.707\end{array}$ & $\begin{array}{l}0.440 \\
0.0424 \\
0.0141\end{array}$ & $\begin{array}{l}11.4 \\
2.40 \\
0.801\end{array}$ & $\begin{array}{l}0.229 \\
0.0481 \\
0.0160\end{array}$ & $\begin{array}{l}0.523 \\
0.113 \\
0.0375\end{array}$ \\
\hline
\end{tabular}


Table 2 Continued

\begin{tabular}{|c|c|c|c|c|c|c|c|}
\hline \multirow[t]{2}{*}{ Energy (keV) } & \multirow[t]{2}{*}{ Temperature $(K)$} & \multirow[t]{2}{*}{ Number of cascades } & \multicolumn{2}{|c|}{$\begin{array}{l}\text { Surviving MD } \\
\text { displacements } \\
\text { (mean / standard } \\
\text { deviation / standard } \\
\text { error) }\end{array}$} & \multicolumn{3}{|c|}{$\begin{array}{l}\text { Clustered interstitials (mean / } \\
\text { standard deviation / standard error) }\end{array}$} \\
\hline & & & Number & per NRT & Number & per NRT & $\begin{array}{l}\text { per MD } \\
\text { surviving } \\
\text { defects }\end{array}$ \\
\hline 5 & 600 & 13 & $\begin{array}{c}19.1 \\
3.88 \\
1.08\end{array}$ & $\begin{array}{l}0.382 \\
0.0777 \\
0.0215\end{array}$ & $\begin{array}{l}9.77 \\
4.09 \\
1.13\end{array}$ & $\begin{array}{l}0.195 \\
0.0817 \\
0.0227\end{array}$ & $\begin{array}{l}0.504 \\
0.187 \\
0.0520\end{array}$ \\
\hline 5 & 900 & 8 & $\begin{array}{l}17.1 \\
2.59 \\
0.915\end{array}$ & $\begin{array}{l}0.343 \\
0.0518 \\
0.0183\end{array}$ & $\begin{array}{l}8.38 \\
1.85 \\
0.653\end{array}$ & $\begin{array}{l}0.168 \\
0.0369 \\
0.0131\end{array}$ & $\begin{array}{l}0.488 \\
0.0739 \\
0.0261\end{array}$ \\
\hline 10 & 100 & 15 & $\begin{array}{c}33.6 \\
5.29 \\
1.37\end{array}$ & $\begin{array}{l}0.336 \\
0.0529 \\
0.0137\end{array}$ & $\begin{array}{l}17.0 \\
4.02 \\
1.04\end{array}$ & $\begin{array}{l}0.170 \\
0.0402 \\
0.0104\end{array}$ & $\begin{array}{l}0.506 \\
0.101 \\
0.0261\end{array}$ \\
\hline 10 & 600 & 8 & $\begin{array}{l}30.5 \\
10.35 \\
3.66\end{array}$ & $\begin{array}{l}0.305 \\
0.104 \\
0.0366\end{array}$ & $\begin{array}{c}18.1 \\
8.46 \\
2.99\end{array}$ & $\begin{array}{l}0.181 \\
0.0846 \\
0.0299\end{array}$ & $\begin{array}{l}0.579 \\
0.115 \\
0.0406\end{array}$ \\
\hline 10 & 900 & 7 & $\begin{array}{c}27.3 \\
5.65 \\
2.14\end{array}$ & $\begin{array}{l}0.273 \\
0.0565 \\
0.0214\end{array}$ & $\begin{array}{c}18.6 \\
6.05 \\
2.29\end{array}$ & $\begin{array}{l}0.186 \\
0.0605 \\
0.0229\end{array}$ & $\begin{array}{l}0.679 \\
0.0160 \\
0.00606\end{array}$ \\
\hline 20 & 100 & 10 & $\begin{array}{c}60.2 \\
8.73 \\
2.76\end{array}$ & $\begin{array}{l}0.301 \\
0.0437 \\
0.0138\end{array}$ & $\begin{array}{r}36.7 \\
6.50 \\
2.06\end{array}$ & $\begin{array}{l}0.184 \\
0.0325 \\
0.0103\end{array}$ & $\begin{array}{l}0.610 \\
0.0630 \\
0.0199\end{array}$ \\
\hline 20 & 600 & 8 & $\begin{array}{c}55.8 \\
5.90 \\
2.09\end{array}$ & $\begin{array}{l}0.281 \\
0.0290 \\
0.0103\end{array}$ & $\begin{array}{c}41.6 \\
5.85 \\
2.07\end{array}$ & $\begin{array}{l}0.211 \\
0.0285 \\
0.0101\end{array}$ & $\begin{array}{l}0.746 \\
0.0796 \\
0.0281\end{array}$ \\
\hline 20 & 900 & 10 & $\begin{array}{c}51.7 \\
9.76 \\
3.09\end{array}$ & $\begin{array}{l}0.259 \\
0.0488 \\
0.0154\end{array}$ & $\begin{array}{c}35.4 \\
8.94 \\
2.83\end{array}$ & $\begin{array}{l}0.177 \\
0.0447 \\
0.0141\end{array}$ & $\begin{array}{l}0.682 \\
0.0944 \\
0.0299\end{array}$ \\
\hline 30 & 100 & 16 & $\begin{array}{c}94.9 \\
13.2 \\
3.29\end{array}$ & $\begin{array}{l}0.316 \\
0.0440 \\
0.0110\end{array}$ & $\begin{array}{c}57.2 \\
11.5 \\
2.88\end{array}$ & $\begin{array}{l}0.191 \\
0.0385 \\
0.00963\end{array}$ & $\begin{array}{l}0.602 \\
0.0837 \\
0.0209\end{array}$ \\
\hline 40 & 100 & 8 & $\begin{array}{r}131.0 \\
12.6 \\
4.45\end{array}$ & $\begin{array}{l}0.328 \\
0.0315 \\
0.0111\end{array}$ & $\begin{array}{c}74.5 \\
15.0 \\
5.30\end{array}$ & $\begin{array}{l}0.186 \\
0.0375 \\
0.0133\end{array}$ & $\begin{array}{l}0.570 \\
0.102 \\
0.0361\end{array}$ \\
\hline 50 & 100 & 9 & $\begin{array}{c}168.3 \\
12.1 \\
4.04\end{array}$ & $\begin{array}{l}0.337 \\
0.0242 \\
0.00807\end{array}$ & $\begin{array}{r}93.6 \\
6.95 \\
2.32\end{array}$ & $\begin{array}{l}0.187 \\
0.0139 \\
0.00463\end{array}$ & $\begin{array}{l}0.557 \\
0.0432 \\
0.0144\end{array}$ \\
\hline 100 & 100 & 10 & $\begin{array}{r}329.7 \\
28.2 \\
8.93\end{array}$ & $\begin{array}{l}0.330 \\
0.0283 \\
0.0089\end{array}$ & $\begin{array}{r}184.8 \\
20.5 \\
6.47\end{array}$ & $\begin{array}{l}0.185 \\
0.0205 \\
0.00650\end{array}$ & $\begin{array}{l}0.561 \\
0.0386 \\
0.0122\end{array}$ \\
\hline 100 & 600 & 20 & $\begin{array}{r}282.4 \\
26.6 \\
5.95\end{array}$ & $\begin{array}{l}0.282 \\
0.0266 \\
0.00595\end{array}$ & $\begin{array}{r}185.5 \\
26.9 \\
6.01\end{array}$ & $\begin{array}{l}0.186 \\
0.0269 \\
0.00601\end{array}$ & $\begin{array}{l}0.656 \\
0.0556 \\
0.0124\end{array}$ \\
\hline 100 & 900 & 18 & $\begin{array}{r}261.0 \\
17.5 \\
4.13\end{array}$ & $\begin{array}{l}0.261 \\
0.0175 \\
0.00413\end{array}$ & $\begin{array}{c}168.7 \\
17.3 \\
4.08\end{array}$ & $\begin{array}{l}0.169 \\
0.0173 \\
0.00408\end{array}$ & $\begin{array}{l}0.646 \\
0.0498 \\
0.0117\end{array}$ \\
\hline 200 & 100 & 9 & $\begin{array}{r}676.7 \\
37.9 \\
12.6\end{array}$ & $\begin{array}{l}0.338 \\
0.0190 \\
0.00632\end{array}$ & $\begin{array}{c}370.3 \\
29.5 \\
9.83\end{array}$ & $\begin{array}{l}0.185 \\
0.0147 \\
0.00491\end{array}$ & $\begin{array}{l}0.548 \\
0.0464 \\
0.0155\end{array}$ \\
\hline
\end{tabular}




\subsubsection{Cascade Evolution and Structure}

The evolution of displacement cascades is similar at all energies, with the development of a highly energetic, disordered core region during the initial, collisional phase of the cascade. Vacancies and interstitials are created in equal numbers, and the number of point defects increases sharply until a peak value is reached. Depending on the cascade energy, this occurs at a time in the range of $0.1-1$ ps. This evolution is illustrated in Figure 5 for a range of cascade energies, where the number of vacancies is shown as a function of the cascade time. Many vacancyinterstitial pairs are in quite close proximity at the time of peak disorder. An essentially athermal process of in-cascade recombination of these close pairs takes place as they lose their kinetic energy. This leads to a reduction in the number of defects until a quasisteady-state value is reached after about 5-10 ps. As interstitials in iron are mobile even at $100 \mathrm{~K}$, further short-term recombination occurs between some vacancy-interstitial pairs that were initially separated by only a few atomic jump distances. Finally, a stage is reached where the remaining point defects are sufficiently well separated that further recombination is unlikely on the time scale (a few hundred picoseconds) accessible by MD. Note that the number of stable Frenkel pair is actually somewhat lower than the value shown in Figure 5 because the values obtained using the effective sphere identification procedure were not corrected to account for the interstitial structure discussed above.

A mechanism known as RCS may help explain some aspects of cascade structure. ${ }^{24,41}$ An RCS can be visualized as an extended defect along a closepacked row of atoms. When the first atom is pushed off its site, it dissipates some energy and pushes a second atom into a third, and so on. When the last atom in this chain is unable to displace another, it is left in an interstitial site with the original vacancy several atomic jumps away. Thus, RCSs provide a mechanism of mass transport that can efficiently separate vacancies from interstitials. The explanation is consistent with the observed tendency for the final cascade state to be characterized by a vacancy-rich central region that is surrounded by a region rich in interstitial-type defects. However, although RCSs are observed, particularly in low-energy cascades, they do not appear to be prominent enough to explain the defect separation observed in higher energy cascades. ${ }^{58}$ Visualization of cascade dynamics indicates that the separation occurs by a more collective motion of multiple atoms, and recent work by Calder and coworkers has identified a shockwave-induced mechanism that leads to the formation of large interstitial clusters at the cascade periphery. ${ }^{80}$ This mechanism will be discussed further in Section 1.11.4.3.1. Coherent displacement events involving many atoms have also been reported by Nordlund and coworkers. ${ }^{81}$

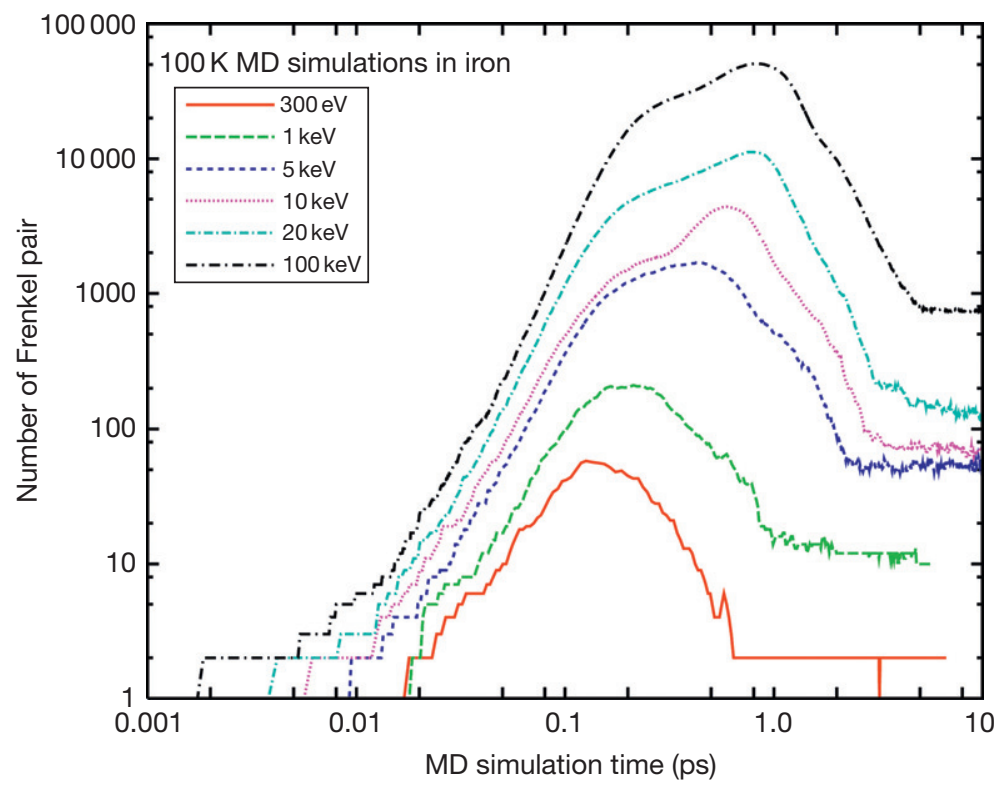

Figure 5 Time evolution of defects formed during displacement cascades. 
Defect production tends to be dominated by a series of simple binary collisions at low PKA energies, while the more collective, cascade-like behavior dominates at higher energies. The structure of typical 1 and $20 \mathrm{keV}$ cascades is shown in Figure 6, where parts (a) and (b) show the peak damage state and (c) and (d) show the final defect configurations. The MD cells contained 54000 and 432000 atoms for the 1 and $20 \mathrm{keV}$ simulations, respectively. Only the vacant lattice sites and interstitial atoms identified by the effective sphere approach described above are shown. The separation of vacancies from interstitials can be seen in the final defect configurations; it is more obvious in the $1 \mathrm{keV}$ cascade because there are fewer defects present. In addition to isolated point defects, small interstitial clusters are also clearly observed in the $20 \mathrm{keV}$ cascade debris in Figure 6(d). In-cascade clustering is discussed further in Section 1.11.4.3.

The morphology of the $20 \mathrm{keV}$ cascade in Figure 6(b) exhibits several lobes which are evidence of a phenomenon known as subcascade formation. ${ }^{82}$ At low energies, the PKA energy tends to be dissipated in a small volume and the cascades appear as compact, sphere-like entities as illustrated by the
$1 \mathrm{keV}$ cascade in Figure 6(a). However, at higher energies, some channeling ${ }^{82,83}$ of recoil atoms may occur. This is a result of the atom being scattered into a relatively open lattice direction, which may permit it to travel some distance while losing relatively little energy in low-angle scattering events. The channeling is typically terminated in a highangle collision in which a significant fraction of the recoil atom's energy is transmitted to the next generation knock-on atom. When significant subcascade formation occurs, the region between high-angle collisions can be relatively defect-free as the cascade develops. This evolution is clearly shown in Figure 7 for a $40 \mathrm{keV}$ cascade, where the branching due to high-angle collisions is observed on a time scale of a few hundreds of femto seconds. One practical implication of subcascade formation is that very high-energy cascades break up into what looks like a group of lower energy cascades. An example of subcascade formation in a $100 \mathrm{keV}$ cascade is shown in Figure 8 where the results of 5 and $10 \mathrm{keV}$ cascades have been superimposed into the same block of atoms for comparison. The impact of subcascade formation on stable defect production will be discussed in the next section.
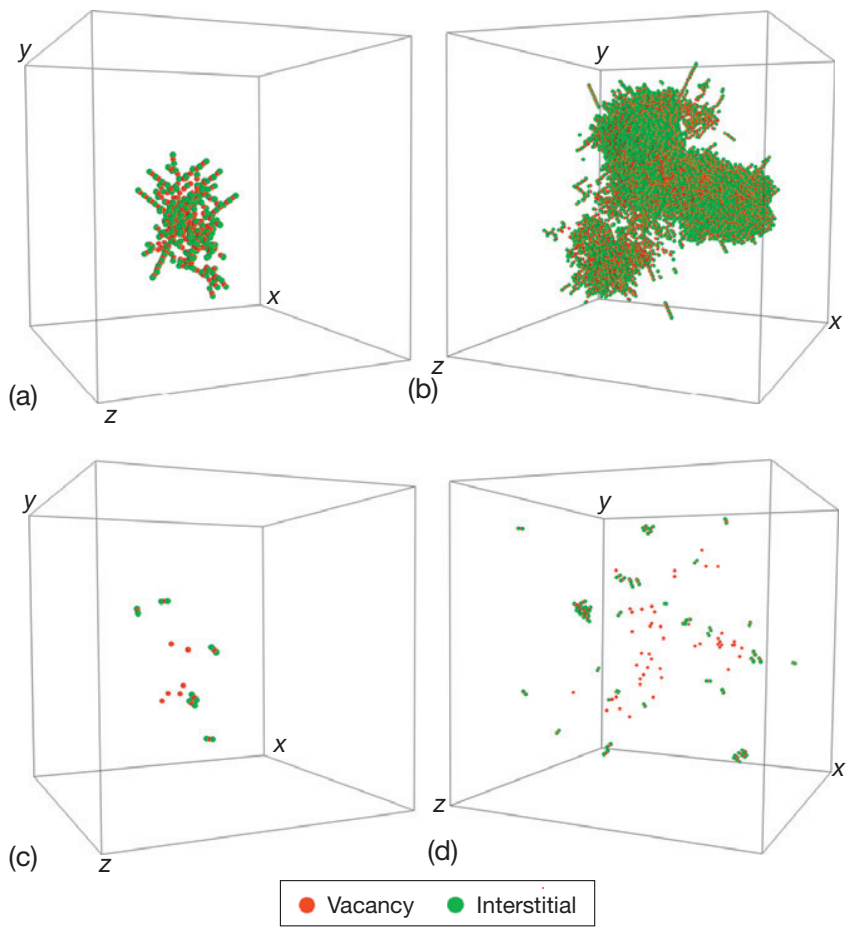

Figure 6 Structure of typical $1 \mathrm{keV}(\mathrm{a}, \mathrm{c})$ and $20 \mathrm{keV}(\mathrm{b}, \mathrm{d})$ cascades. Peak damage state is shown in $(\mathrm{a}$ and $\mathrm{b})$ and the final stable defect configuration is shown in (c and d). 

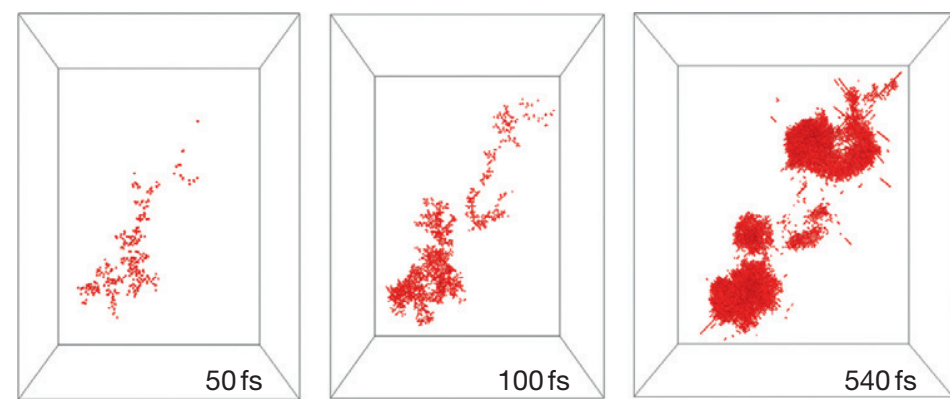

Figure 7 Evolution of a $40 \mathrm{keV}$ cascade in iron at $100 \mathrm{~K}$, illustrating subcascade formation.

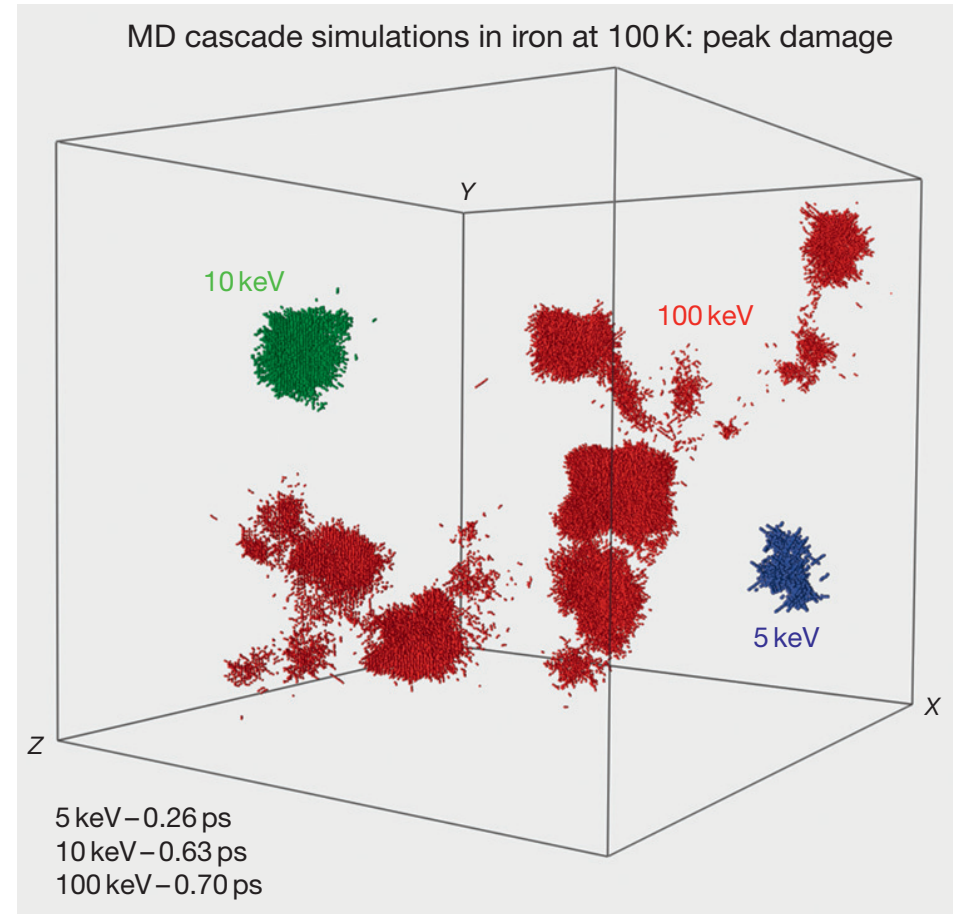

Figure 8 Energy dependence of subcascade formation.

\subsubsection{Stable Defect Formation}

Initial work of Bacon and coworkers indicated that the number of stable displacements remaining at the end of a cascade simulation, $N_{\mathrm{D}}$, exhibited a powerlaw dependence on cascade energy. ${ }^{84}$ For example, their analysis of iron cascade simulations between 0.5 and $10 \mathrm{keV}$ at $100 \mathrm{~K}$ showed that the total number of surviving point defects could be expressed as

$$
N_{\mathrm{D}}=5.67 E_{\mathrm{MD}}^{0.779}
$$

where $E_{\mathrm{MD}}$ is given in kiloelectronvolts. This relationship is not followed below about $0.5 \mathrm{keV}$ because true cascade-like behavior does not occur at these low energies. Subsequent work by Stoller ${ }^{64-67}$ indicated that $N_{\mathrm{D}}$ also begins to deviate from this energy dependence above $20 \mathrm{keV}$ when extensive subcascade formation occurs. This is illustrated in Figure 9(a) where the values of $N_{\mathrm{D}}$ obtained in cascade simulations at $100 \mathrm{~K}$ is plotted as a function of cascade energy. At each energy, the data point is an average of between 7 and 26 cascades, and the error bars indicate the standard error of the mean. It appears that three well-defined regions with different energy dependencies exist. A power-law fit to the points in each energy region is also shown in Figure 9(a). The best-fit exponent in the absence of true cascade conditions below $0.5 \mathrm{keV}$ is 0.485 . From 0.5 to 

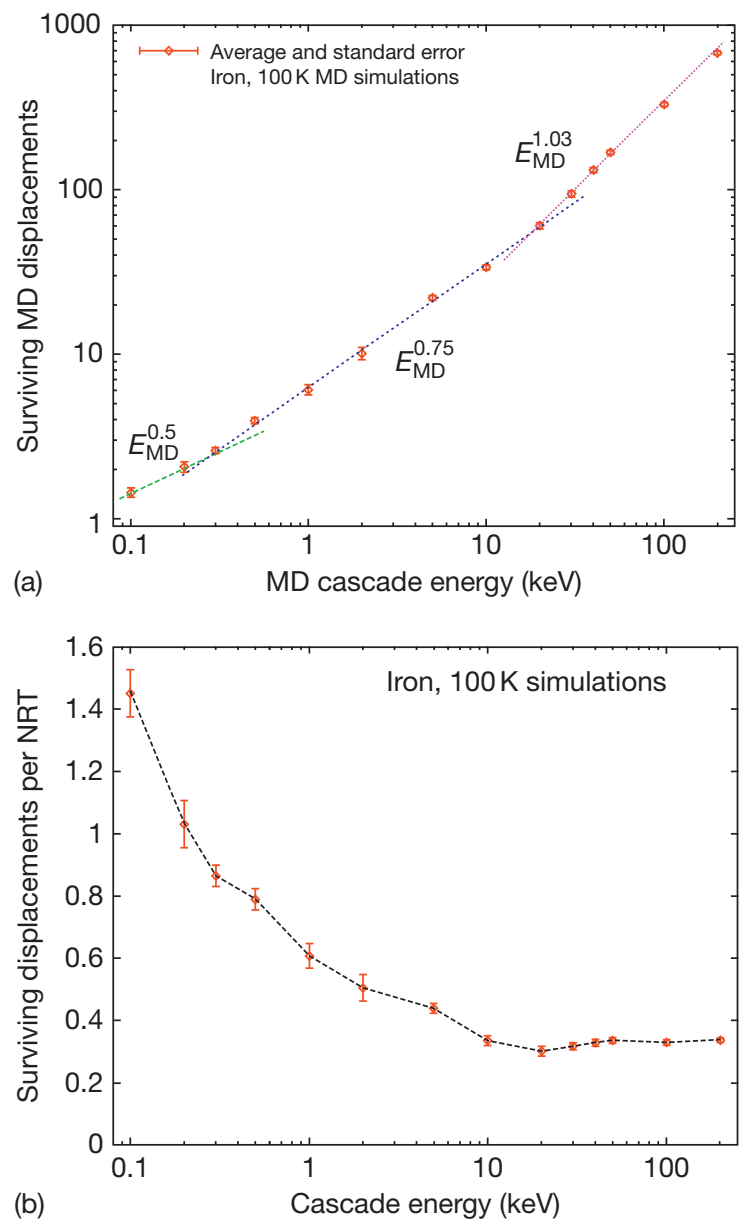

Figure 9 Cascade energy dependence of stable point defect formation in iron MD cascade simulations at $100 \mathrm{~K}$ : (a) total number of interstitials or vacancies and (b) ratio of MD defects to NRT displacements. Data points indicate mean values at each energy, and error bars are standard error of the mean.

$20 \mathrm{keV}$, the exponent is 0.75 . This is marginally lower than the value in eqn [2], possibly because the $20 \mathrm{keV}$ data were used in the current fitting. An exponent of 1.03 was found in the range above $20 \mathrm{keV}$, which is dominated by subcascade formation. Only in the highest energy range do the MD results approach the linear energy dependence predicted by the NRT model. The range of plus or minus one standard error is barely detectable around the data points, indicating that the change in slope is statistically significant.

The data from Figure 9(a) are replotted in Figure 9(b) where the number of surviving displacements is divided by the NRT displacements at each energy. The rapid decrease in this MD defect survival ratio at low energies was first measured in 1978 and is well known. ${ }^{57,85}$ The error bars again reflect the standard error and the dashed line through the points is only a guide to the eye. The MD/NRT ratio is greater than 1.0 at the lowest values of $E_{\mathrm{MD}}$, indicating that the NRT formulation underestimates defect production in this energy range. This is consistent with the low-energy (near threshold) simulations preferentially producing displacements in the 'easy' directions. ${ }^{26}$ The actual displacement threshold varies with crystallographic direction and is as low as $\sim 19 \mathrm{eV}$ in the [100] direction. ${ }^{20,84}$ Thus, using the recommended average value of $40 \mathrm{eV} E_{\mathrm{d}}$ in eqn [2] predicts fewer defects at low energies. The average value is more appropriate for the higher energy events where true cascade-like behavior occurs. In the cascade-dominated regime, the defect density within the cascade increases with energy. Although many more defects are produced, their close proximity leads to a higher probability of in-cascade recombination and a lower defect survival fraction.

The surviving defect fraction shows a slight increase as the cascade energy increases above 20, and the indicated standard errors make it arguable that the increase is statistically significant. If significant, the increase appears to be associated with subcascade formation, which becomes prominent above $10-20 \mathrm{keV}$. In the channeling regions between the high-angle collisions that produce the subcascades shown in Figures 7 and 8, the moving atom loses energy in many low-angle scattering events that produce low-energy recoils. These are essentially like low-energy cascades, which have higher-than-average defect survival fractions (Figure 9). These events could contribute to the incremental increase in defect survival at the highest energies. The average defect survival fraction of $\sim 0.3$ NRT shown for cascade energies greater than about $10 \mathrm{keV}$ is consistent with values of Frenkel pair formation obtained from resistivity change measurements following low-temperature neutron irradiation and ion irradiation. ${ }^{26,27,57,85}$

The effect of irradiation temperature is shown in Figure 10, which compares the defect survival fractions obtained from simulations at 100,600, and $900 \mathrm{~K}$. Although it is difficult to discern a consistent effect of temperature between the 600 and $900 \mathrm{~K}$ data points, the defect survival fraction at $100 \mathrm{~K}$ is always somewhat greater than at either of the two higher temperatures. A similar result for iron was reported in Bacon and coworkers. ${ }^{84}$ In addition to an interest in radiation temperature itself, the effect of temperature is relevant to the 
simulations presented here because no thermostat was applied to the simulation cell to control temperature. As mentioned above, the energy introduced by the PKA will lead to some heating if the simulation cell temperature is not controlled by a thermostat. For example, in a $1 \mathrm{keV}$ cascade simulation with 54000 atoms, the average temperature rise will be about $140 \mathrm{~K}$ when all the kinetic energy of the PKA is distributed in the system. This change in temperature should be more significant at $100 \mathrm{~K}$ than at higher temperatures. The fact that defect survival at 600 and $900 \mathrm{~K}$ is lower than at $100 \mathrm{~K}$ suggests that the $100 \mathrm{~K}$ results may be

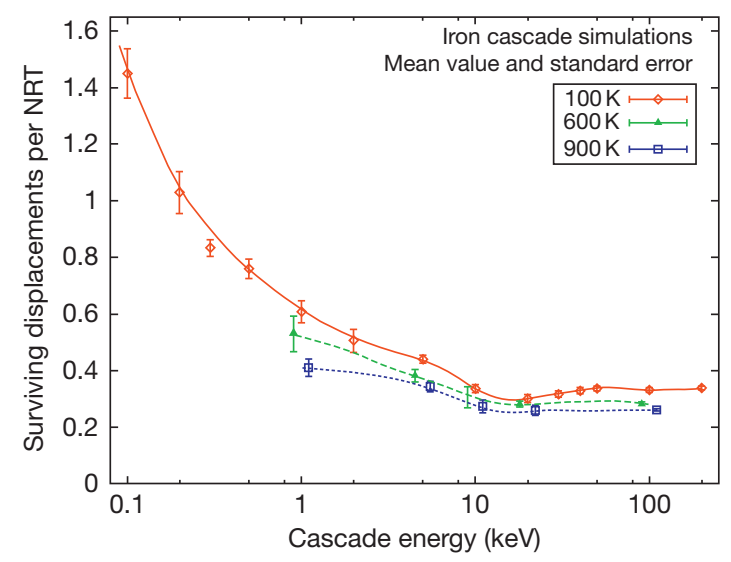

Figure 10 Temperature dependence of stable defect formation in MD simulations: ratio of MD defects to NRT displacements. somewhat biased toward lower survival values by the PKA-induced heating. This is in agreement with the effect of temperature reported by Gao and coworkers ${ }^{77}$ in their study of 2 and $5 \mathrm{keV}$ cascades with a hybrid MD model that extracted heat from the simulation cell. On the other hand, the difference between the 100 and $600 \mathrm{~K}$ results is not large, so the effect of $\sim 200 \mathrm{~K}$ of cascade-induced heating may be modest.

A simple assessment of this cascade-induced heating was carried out using $10 \mathrm{keV}$ cascades at $100 \mathrm{~K}$. Two independent sets of simulations were carried out, seven simulations in a cell of $128 \mathrm{k}$ atoms and eight simulations in a cell of $250 \mathrm{k}$ atoms. A $10 \mathrm{keV}$ cascade will raise the average temperature by 604 and $309 \mathrm{~K}$, respectively, for these two cell sizes. The results of these simulations are summarized in Figure 11, where the parameters plotted are the surviving defect fraction (per NRT), the fraction of interstitials in clusters (per NRT), and the fraction of interstitials in clusters (per surviving MD defect). In each case, the range of values for the two populations are shown, along with their respective mean values with the standard error indicated. The mean and standard error for the combined data sets is also shown. Although the heating differed by a factor of two, it is clear that the defect survival fraction is essentially identical for both populations. There is a slight trend in the interstitial clustering results, which indicates that a higher temperature (due to a smaller number of atoms) promotes interstitial clustering. This is consistent with the results that will be discussed below.

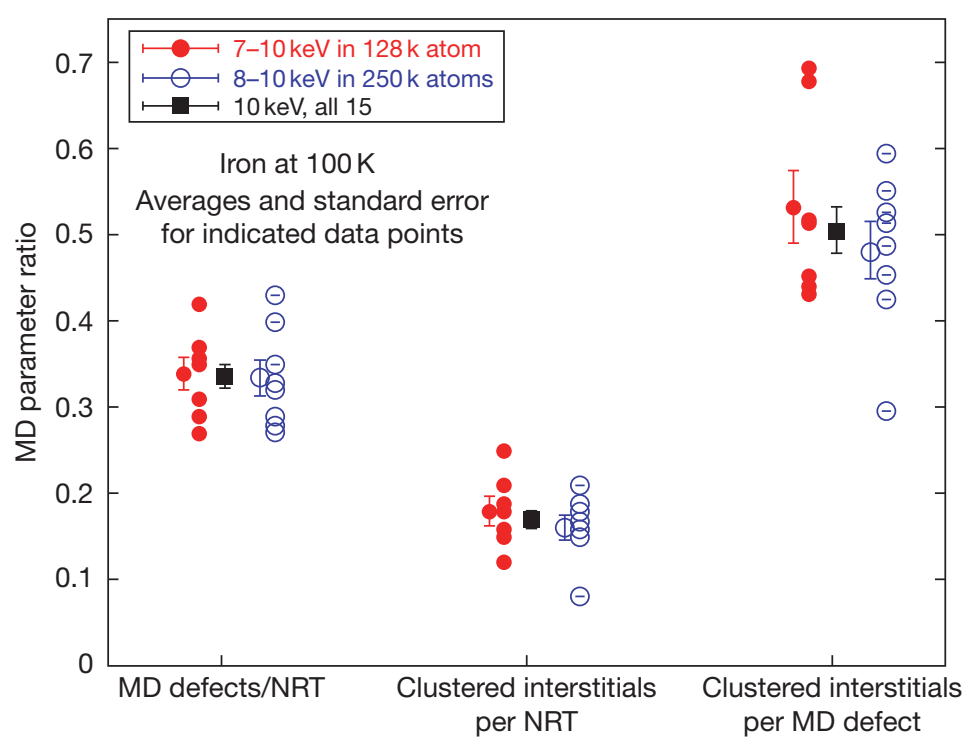

Figure 11 Effect of cascade heating on defect formation in $10 \mathrm{keV}$ cascades at $100 \mathrm{~K}$. 


\subsubsection{In-cascade Clustering of Point Defects}

Among the features visible in the two cascades shown in Figure 6 are a number of small interstitial clusters. For example, the cascade debris from the $1 \mathrm{keV}$ cascade in Figure 6(c) contains only seven stable interstitials, but five of them $(71 \%)$ are in clusters: one di-interstitial and one tri-interstitial. This tendency for point defects to cluster is characteristic of energetic displacement cascades, and it differentiates neutron and ion irradiation from typical 0.5 to $1 \mathrm{MeV}$ electron irradiation, which primarily produces only isolated Frenkel pair defects. The differences between in-cascade vacancy and interstitial clustering discussed below, and the fact that their migration behavior is also quite different, have a profound influence on radiation-induced microstructural evolution at longer times. This impact of point defect clusters on microstructural evolution is discussed in detail in Chapter 1.13, Radiation Damage Theory.

\subsection{Interstitial clustering}

The dependence of in-cascade interstitial clustering on cascade energy is shown in Figure 12 for simulation temperatures of 100,600 , and $900 \mathrm{~K}$, where the average number of interstitials in clusters of size two or larger at each energy has been divided by the total number of surviving interstitials in part (a), and by the number of displaced atoms predicted by the NRT model for that energy in part (b). The data points and error bars in Figure 12 indicate the mean and standard error at each energy. The error bars can be used to make two significant comments. First, the relative scatter is much higher at lower energies, which is similar to the case of defect survival shown in Figure 10. Second, comparing again with Figure 10, the standard errors about the mean for interstitial clustering are greater at each energy than they are for defect survival.

The fact that the interstitial clustering fraction exhibits greater variability between cascades at a given energy than does defect survival is essentially related to the variety of defect configurations that are possible. A given amount of kinetic energy tends to produce a given number of stable point defects; this simple observation is embedded in the NRT model, that is, the number of predicted defects is linear in the ratio of the energy available to the energy per defect. However, any specific number of point defects can be arranged in many different ways.
At the lowest energies, where relatively few defects are created, some cascades produce no interstitial clusters and this is primarily responsible for the larger error bars at these energies. The average fraction of interstitials in clusters is about $20 \%$ of the NRT displacements above $5 \mathrm{keV}$, which corresponds to about $60 \%$ of the total surviving interstitials. Although it is not possible to discern a systematic effect of temperature below $10 \mathrm{keV}$, there is a trend toward greater clustering with increasing temperature at higher energies. This can be more clearly seen in Figure 12(a) where the ratio of clustered interstitials to surviving interstitials is shown, and in the high-energy values in Table 2. This effect of temperature on interstitial clustering in these adiabatic simulations is consistent with the observations of Gao and coworkers ${ }^{77}$ mentioned above, that is, they found that the interstitial clustering fraction increases with temperature.

The interstitial cluster size distributions exhibit a consistent dependence on cascade energy and temperature as shown in Figure 13 (where a size of 1 denotes the single interstitial). The cascade energy dependence at $100 \mathrm{~K}$ is shown in Figure 13(a), where the size distributions from 10 and $50 \mathrm{keV}$ are included. The influence of cascade temperature is shown for $10 \mathrm{keV}$ cascades in Figure 13(b), and for $20 \mathrm{keV}$ cascades in Figure 13(c). All interstitial clusters larger than size 10 are combined into a single class in the histograms in Figure 13. The interstitial cluster size distribution shifts to larger sizes as either the cascade energy or temperature increases. An increase in the clustering fraction at the higher temperatures is most clearly seen as a decrease in the number of mono-interstitials. Comparing Figures 13(b) and 13(c) demonstrates that the temperature dependence increases as the cascade energy increases. The largest interstitial cluster observed in these simulations was contained in a $20 \mathrm{keV}$ cascade at $600 \mathrm{~K}$ as shown in Figure 14. This large cluster was composed of 33 interstitials ( $<111>$ crowdions), and exhibited considerable mobility via what appeared to be a $1 \mathrm{D}$ glide in a $<111>$ direction. ${ }^{64,66}$

Although the number of point defects produced and the fraction of interstitials in clusters was shown to be relatively independent of neutron energy spectrum, ${ }^{82}$ the increase in the number of large clusters at higher energies suggested that the in-cascade cluster size distributions may exhibit more sensitivity to neutron energy spectrum than did these other parameters. At $100 \mathrm{~K}$, there are no interstitial clusters larger than 8 for cascade energies of $10 \mathrm{keV}$ or 

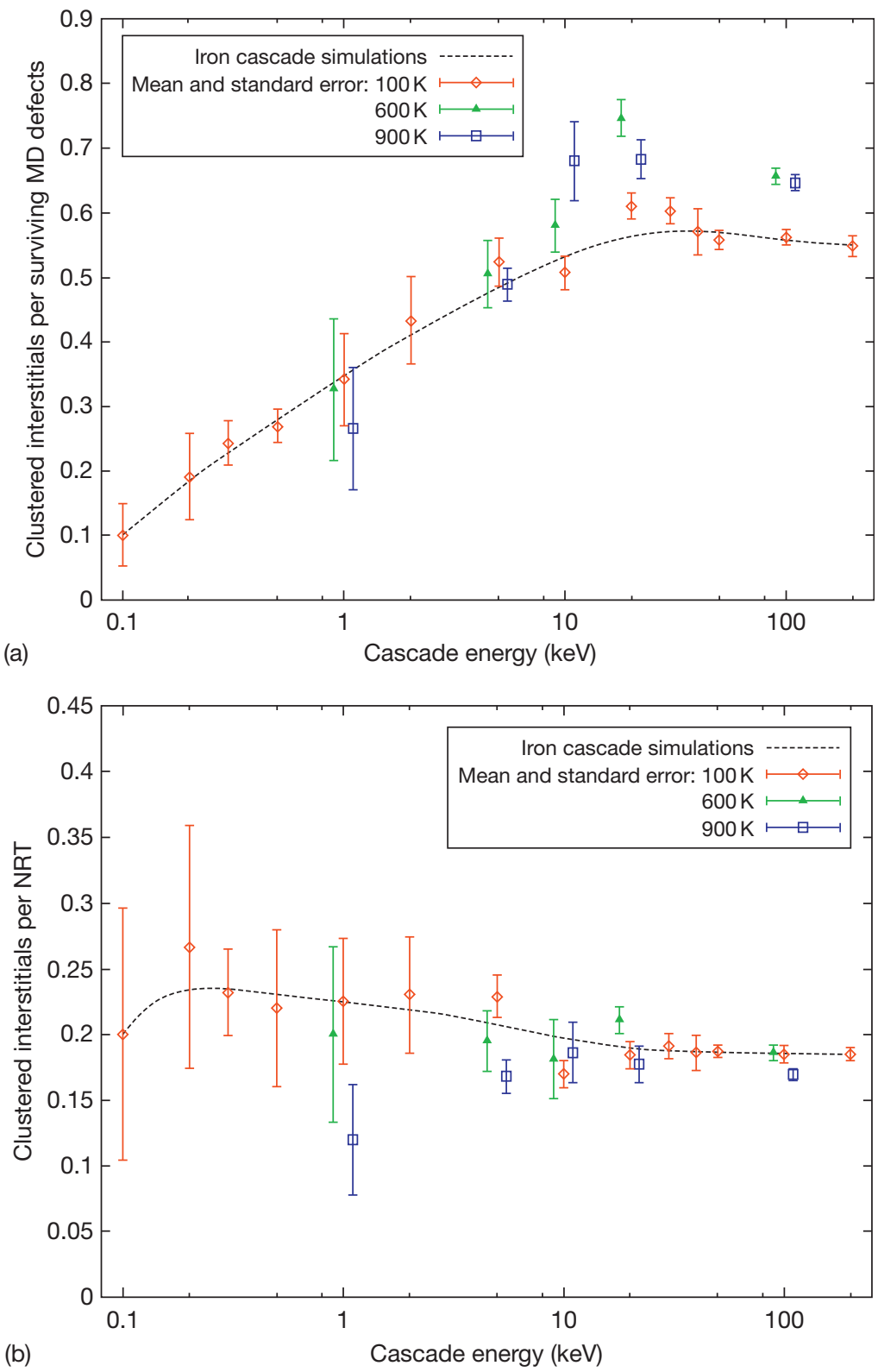

Figure 12 Fraction of surviving interstitials contained in clusters at $100 \mathrm{~K}$; the fraction in (a) is relative to the total number of MD defects created and in (b) is relative to the NRT displacements.

less. Therefore, the fraction of interstitials in clusters of 10 or more was chosen as an initial parameter for evaluation of the size distributions. This partial interstitial clustering fraction is shown in Figure 15. As the large clusters are relatively uncommon, the fraction of interstitials contained in them is correspondingly small. This leads to the relatively large standard errors shown in the figure. However, it is clear that the energy dependence of the formation of these large clusters is much stronger than simply the total fraction of interstitials in clusters. Infrequent large clusters such as the 33-interstitial cluster shown in Figure 14 play a significant role in the sharp increase in this clustering fraction observed between 100 and $600 \mathrm{~K}$ for the $20 \mathrm{keV}$ cascades.

One unusual observation reported by Wooding and coworkers ${ }^{60}$ and Gao and coworkers ${ }^{86}$ was that some of the interstitial clusters exhibited a complex 3D morphology rather than collapsing into planar dislocation loops which are expected to have lower 

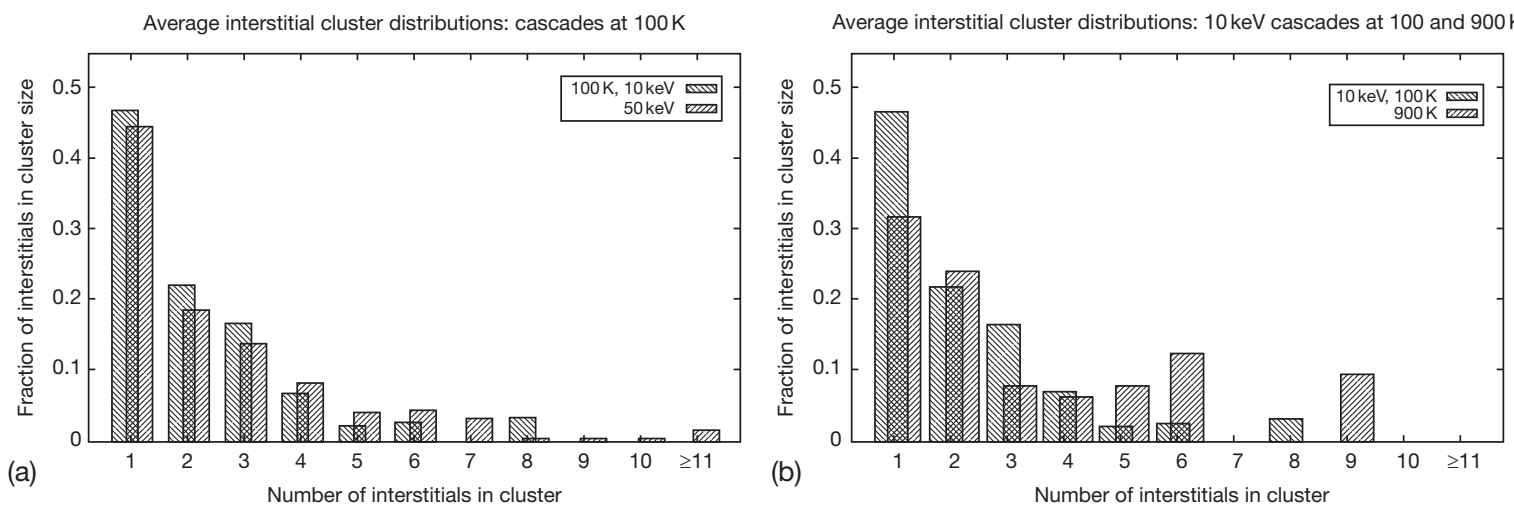

Average interstitial cluster distributions: $20 \mathrm{keV}$ cascades at 100 and $600 \mathrm{~K}$

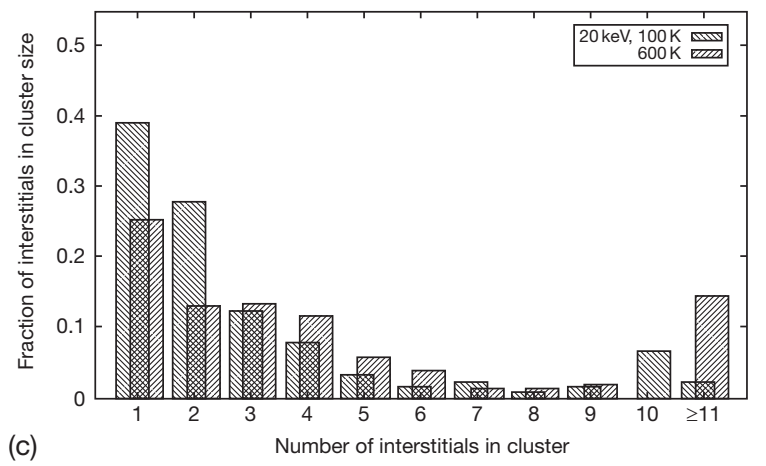

Figure 13 Fractional size distributions of interstitial clusters formed directly within the cascade, comparison of (a) 10 and $50 \mathrm{keV}$ cascades at $100 \mathrm{~K}$, (b) $10 \mathrm{keV}$ cascades at 100 and $900 \mathrm{~K}$, and (c) $20 \mathrm{keV}$ cascades at 100 and $600 \mathrm{~K}$.

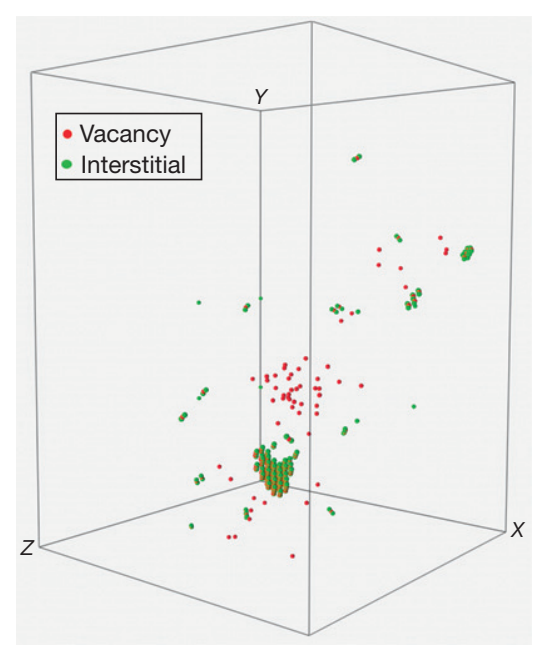

Figure 14 Residual defects at $\sim 30 \mathrm{ps}$ from a $20 \mathrm{keV}$ cascade at $600 \mathrm{~K}$ containing a 33 -interstitial cluster.

energy. Similar clusters have been seen in materials such as copper, although they appear to be less frequent in copper. ${ }^{54}$ The existence of such clusters has been confirmed with interatomic potentials that were developed more recently and with ab initio

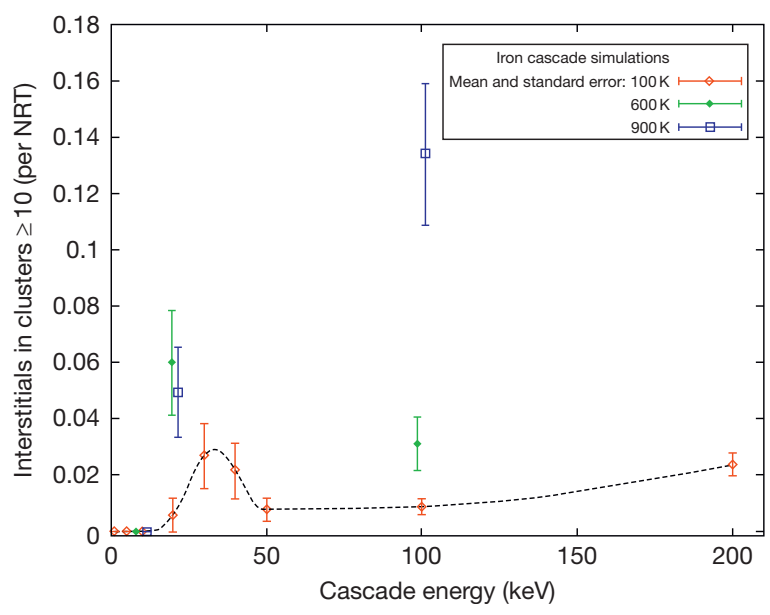

Figure 15 Cascade energy dependence of interstitials contained in clusters of 10 or more: clustered interstitials divided by NRT displacements.

calculations. ${ }^{87}$ Representative examples of these clusters are shown in Figure 16, where a ring-like four-interstitial cluster is shown in (a) and a fiveinterstitial cluster is shown in (b). Unlike the mobile clusters that are composed of [111] crowdions such 


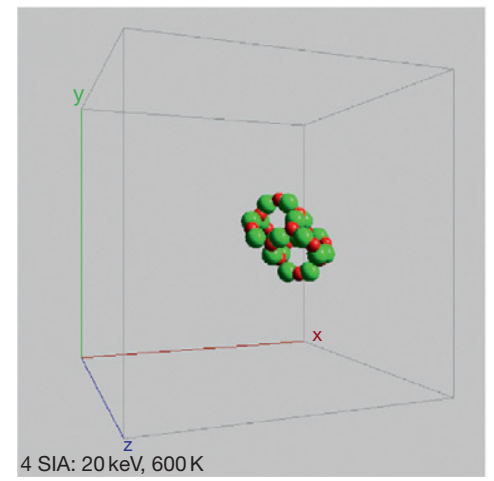

(a)

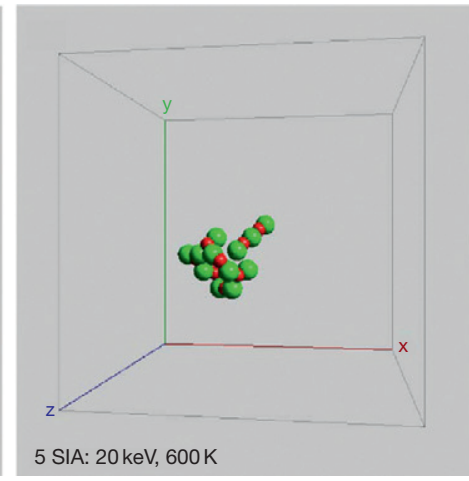

(b)

Figure 16 Two examples of sessile interstitial configurations formed in $20 \mathrm{keV}, 600 \mathrm{~K}$ displacement cascades: clusters in (a) and (b) consist of 4 and 5 SIA, respectively (cf. the glissile cluster configuration in Figure 14).

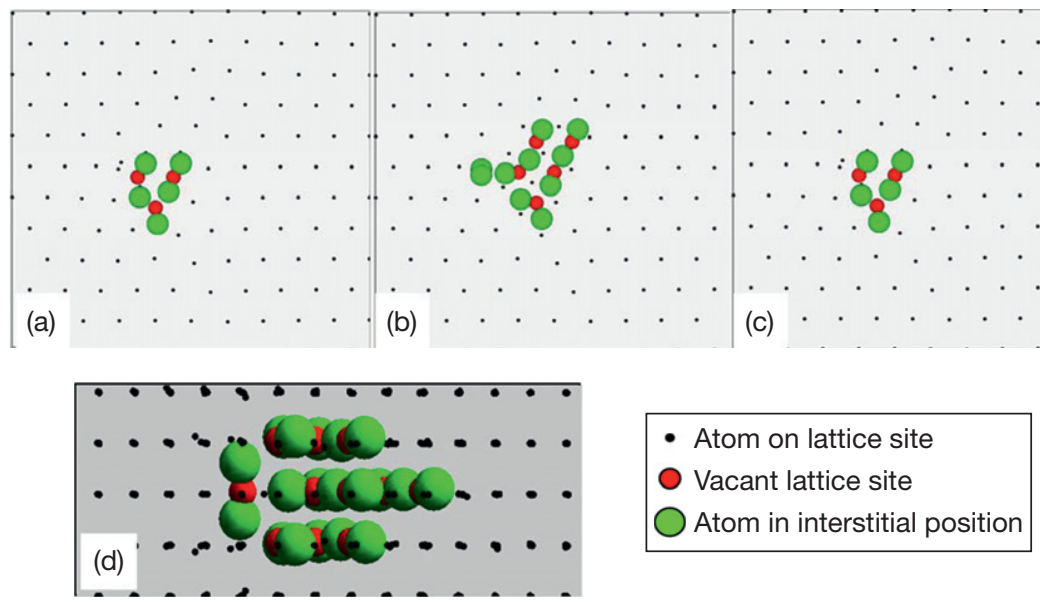

Figure 17 Three-dimensional sessile interstitial cluster in $10 \mathrm{keV}, 100 \mathrm{~K}$ cascade: (a) [010] projection normal to five adjacent $\{110\}$ planes, $(b-d)$ projections through three of the $\{110\}$ planes.

as the one shown in Figure 14, the SIA clusters in Figure 16 are not mobile. As such, they have the potential for long lifetimes in the microstructure and may act as nucleation sites for larger interstitial-type defects. Figure 17 shows a somewhat larger sessile cluster containing eight SIA. This particular cluster was examined in detail by searching a large number of low-order crystallographic projections in an attempt to find a projection in which it would appear as a loop. Such a projection could not be found. Rather, the cluster was clearly 3D with a single di-, tri-, and di-interstitial on adjacent, close-packed (110) planes as shown in the figure. The eighth interstitial is a [110] dumbbell that lies perpendicular to the others and on the left side in Figure 17(a). Figure $16(b-d)$ are [101] projections through the three center (101) planes in Figure 17(a).
It is possible that the typical $10-15$ ps MD simulation was not sufficient for the cluster to reorient and collapse. To examine this possibility, the simulation time of a $10 \mathrm{keV}$ cascade at $100 \mathrm{~K}$ that contained a similar eight SIA cluster was continued up to $100 \mathrm{ps.}$ Very little cluster restructuring was seen over the time from 10 to $100 \mathrm{ps}$. In fact, the cluster had coalesced into nearly its final configuration by 10 ps. Gao and coworkers ${ }^{86}$ carried out a more systematic investigation of sessile cluster configurations with extended simulations at 300 and $500 \mathrm{~K}$. They found that many sessile clusters had converted to glissile within a few hundred picoseconds, but at least one eight SIA cluster remained sessile for $\sim 500$ ps even after aging at temperatures up to $1500 \mathrm{~K}$. Given the impact that stable sessile clusters would have on the longer timescale microstructural evolution as 
discussed in Chapter 1.13, Radiation Damage Theory, further research is needed to characterize the long-term evolution of cascade-created point defect clusters. It is significant to point out that the conversion of glissile SIA clusters into sessile clusters has also been observed. For example, in a $20 \mathrm{keV}$ cascade at $100 \mathrm{~K}$, a glissile eight SIA cluster was trapped and converted into a sessile nine SIA cluster when it reacted with a single [110] dumbbell. The simulation was continued for more than 200 ps and the cluster remained sessile.

The mechanism responsible for interstitial clustering has not been fully understood. For example, it has not been possible to determine whether the motion and agglomeration of individual interstitials and small interstitial clusters during the cascade event contributes to the formation of the larger clusters that are observed at the end of the event. Alternate clustering mechanisms in the literature include the suggestion by Diaz de la Rubia and Guinan ${ }^{88}$ that large clusters could be produced by a loop punching mechanism. Nordlund and coworkers ${ }^{62}$ proposed a 'liquid isolation' model in which solidification of a melt zone isolates a region with excess atoms.

However, a new mechanism has recently been elucidated by Calder and coworkers, ${ }^{80}$ which seems to explain how both vacancy and interstitial clusters are formed, particularly the less frequent large clusters. Their analysis of cluster formation followed an investigation of the effects of PKA mass and energy, which demonstrated that the probability of producing large vacancy and SIA clusters increases as these parameters increase. ${ }^{89}$ The conditions of this study produced a unique dataset that motivated the effort to unravel how the clusters were produced. They developed a detailed visualization technique that enabled them to connect the individual displacements of atoms that resulted in defect formation by comparing the start and end positions of atoms in the simulation cell. This defined a continuous series of links between each vacancy and interstitial that were ultimately produced by a chain of displacements. These chains could be displayed in what are called lines of 'spaghetti. ${ }^{80}$ Regions of tangled spaghetti define a volume in which atoms are highly agitated and a certain fraction of which are displaced. Stable interstitials and interstitial clusters are observed on the surface in this volume.

From their analysis of cascade development and the final damage state, Calder and coworkers were able to demonstrate a correlation between the production of large SIA clusters and a process taking place very early in the development of a cascade. Specifically, they established a direct connection between such clusters and the formation of a hypersonic recoil atom that passed through the supersonic pressure wave created by the initiation of the cascade. This highly energetic recoil may create a subcascade and a secondary supersonic shockwave at an appropriate distance from the primary shockwave. In this case, SIA clusters tend to be formed at the point where the primary and secondary shockwaves interfere with one another. This process is illustrated in Figure 18. ${ }^{80}$ Atoms may be transferred from the primary shockwave volume into the secondary shockwave volume, creating an interstitial supersaturation in the latter and a vacancy supersaturation in the former. In this case, the mechanism of creating large SIA clusters early in the cascade process correspondingly leads to the formation of large vacancy clusters by the end of the thermal spike phase, that is, after several picoseconds. It is notable that the location of the SIA cluster is determined well before the onset of the thermal spike phase, by about 0.1 ps. Calder's spaghetti analysis provides the opportunity for improved definition of parameters such as cascade volume and energy density; the interested reader is directed to Calder and coworkers ${ }^{80}$ for more details.

\subsection{Vacancy clustering}

As discussed elsewhere, ${ }^{59,63,65}$ in-cascade vacancy clustering in iron is quite low $(\sim 10 \%$ of NRT) when a NN criterion for clustering is applied. This was identified as one of the differences between iron and copper in the comparison of these two materials reported by Phythian and coworkers. ${ }^{59}$ However, when the coordinates of the surviving vacancies in 10,20 , and $40 \mathrm{keV}$ cascades were analyzed, clear spatial correlations were observed. Peaks in the distributions of vacancy-vacancy separation distances were obtained for the second and fourth NN locations. ${ }^{64}$ These radial distributions are shown in Figure 19. Similar results were obtained from the analysis of the vacancy distributions in higher energy cascades at 100 and $600 \mathrm{~K}$. The peak observed for vacancies in second $\mathrm{NN}$ locations is consistent with the di-vacancy binding energy being greater for second NN $(0.22 \mathrm{eV})$ than for first NN $(0.09 \mathrm{eV}){ }^{90}$ The reason for the peak at fourth NN is presumably related to this also since two vacancies that are second $\mathrm{NN}$ to a given vacancy would be fourth NN. In addition, work discussed by Djurabekova and coworkers ${ }^{91}$ indicates that there is a small binding energy between two vacancies at the fourth $\mathrm{NN}$ distance. 
(a)
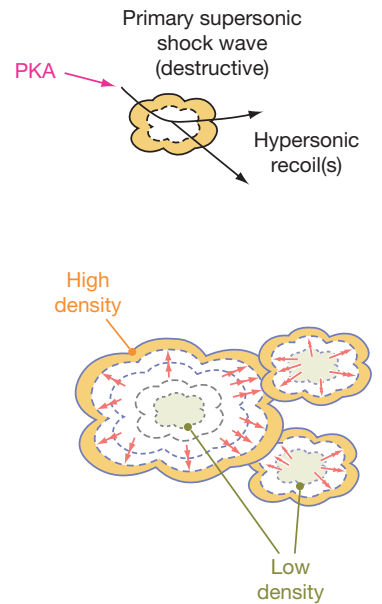

(c)

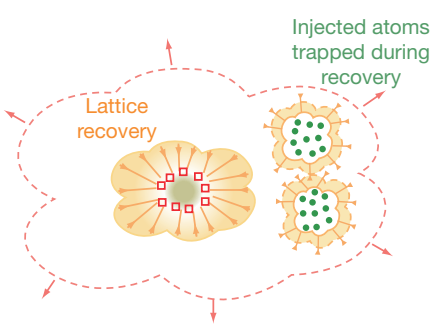

(e)

(f)

(b)

(d)
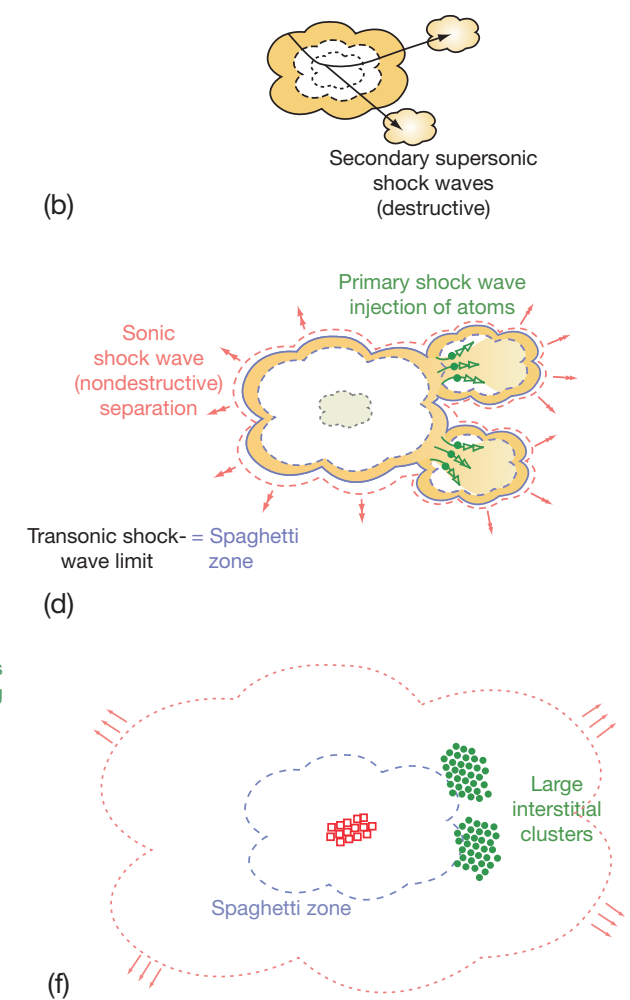

Figure 18 Schematic representation of cascade development leading to the formation of interstitial and vacancy clusters formation. Reproduced from Calder, A. F.; Bacon, D. J.; Barashev, A. V.; Osetsky, Yu. N. Phil. Mag. 2010, 90, 863-884.

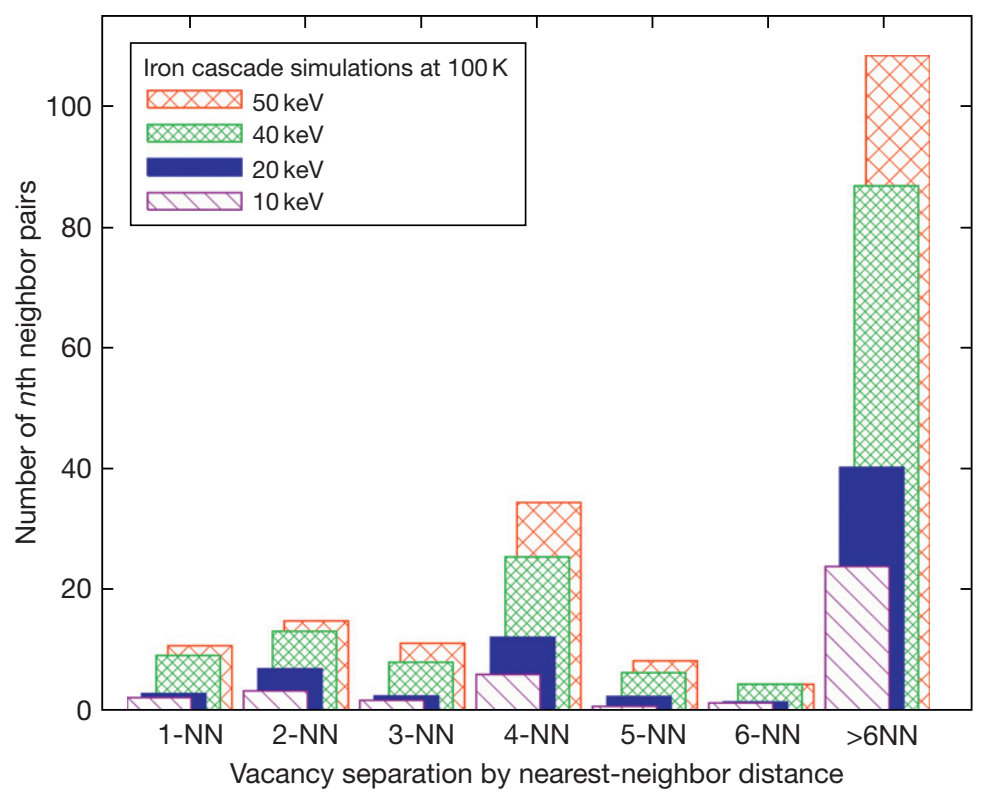

Figure 19 Spatial correlation of all vacancies observed in 10, 20, 40, and $50 \mathrm{keV}$ cascades at $100 \mathrm{~K}$.

An example of a locally vacancy-rich region in a $50 \mathrm{keV}, 100 \mathrm{~K}$ cascade is shown in Figure 20, where the region around a collection of 14 vacancies has been extracted from the larger simulation cell. This appears to be a nascent or uncollapsed vacancy cluster. Each of the vacancies has at least one other 
vacancy within the fourth $\mathrm{NN}$ spacing of $1.66 a_{0}$, where $a_{0}$ is the iron lattice parameter. The 'cluster' is shown in two views: a $3 \mathrm{D}$ perspective view and an orthographic projection $(-x)$ in Figure 20. Such an arrangement of vacancies is similar to some of the vacancy clusters observed by Sato and coworkers in field ion microscope images of irradiated tungsten. ${ }^{92}$ Since the time period of the MD simulations is too short to allow vacancies to jump ( $<100 \mathrm{ps})$, the possibility that these closely correlated vacancies might collapse into clusters over somewhat longer times has

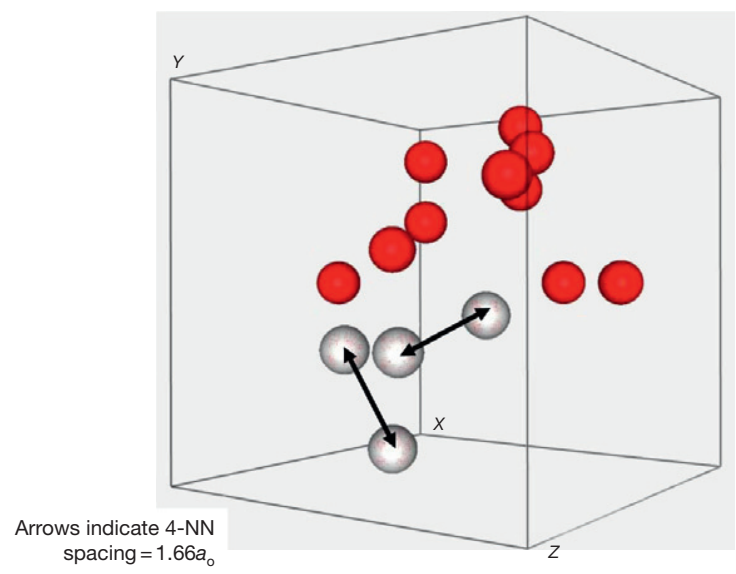

Figure 20 Typical uncollapsed or nascent vacancy cluster from $50 \mathrm{keV}$ cascade at $100 \mathrm{~K}$; 14 vacancies are contained, each of which is within the fourth nearest-neighbor distance $\left(1.66 a_{0}\right)$. been investigated using MC simulations. The vacancy coordinates at the end of the MD simulations were extracted and used as the starting configuration in MC cascade annealing simulations. The expectation of vacancy clustering was confirmed in the MC simulations, where many of the isolated vacancies had clustered within $70 \mathrm{~ms}^{90,93}$

The energy and temperature dependence of in-cascade vacancy clustering as a fraction of the NRT displacements is shown in Figure 21 for cascade energies of $10-50 \mathrm{keV}$. Results are shown for clustering criteria of first, second, third, and fourth NN. A comparison of Figure 21 and Figure 12 demonstrates that in-cascade vacancy clustering in iron remains lower than that of interstitials even when the fourth NN criterion is used. This is consistent with the experimentally observed difficulty of forming visible vacancy clusters in iron as discussed by Phythian and coworkers, ${ }^{59}$ and the fact that only relatively small vacancy clusters are found in positron annihilation studies of irradiated ferritic alloys. ${ }^{94}$ However, it should be pointed out that work with more recently developed iron potentials finds less difference between vacancy and interstitial clustering. ${ }^{74}$ The cascade energy dependence of vacancy clustering is similar to that of interstitials; there is essentially zero clustering at the lowest energies but it rapidly increases with cascade energy and is relatively independent of energy above $\sim 10 \mathrm{keV}$. However, vacancy clustering decreases as the temperature increases,

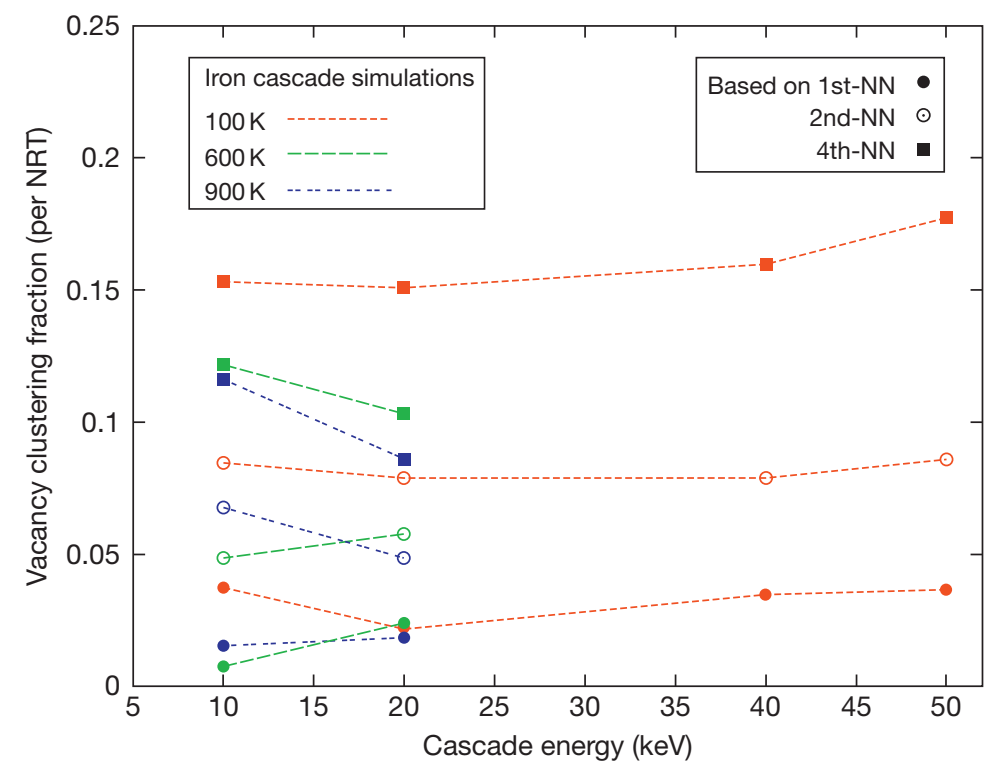

Figure 21 Cascade energy dependence of vacancy clustering: clustered vacancies divided by NRT displacements. Data points indicate mean values, and error bars are standard error of the mean. 
which is consistent with vacancy clusters being thermally unstable.

Fractional vacancy cluster size distributions are shown in Figure 22, for which the fourth NN clustering criterion has been used. Figure 22(a) illustrates that the vacancy cluster size distribution shifts to larger sizes as the cascade energy increases from 10 to $50 \mathrm{keV}$. This is similar to the change shown for interstitial clusters in Figure 13(a). There is a corresponding reduction in the fraction of single vacancies. However, as mentioned above, the effect of cascade temperature shown in Figure 22(b) and 22(c) is the opposite of that observed for interstitials. The magnitude of the temperature effect on the vacancy cluster size distributions also appears to be weaker than in the case of interstitial clusters. The fraction of single vacancies increases and the size distribution shifts to smaller sizes as the temperature increases from 100 to $900 \mathrm{~K}$ for the $10 \mathrm{keV}$ cascades, and from 100 to $600 \mathrm{~K}$ for $20 \mathrm{keV}$ cascades. Similar to the case of interstitial clusters, the effect of temperature seems to be greater at $20 \mathrm{keV}$ than at $10 \mathrm{keV}$.

\subsubsection{Secondary Factors Influencing Cascade Damage Formation}

The results of simulations such as those presented above should not be viewed as being quantitatively accurate. As already mentioned, subtle changes in the fitting of the interatomic potential can alter the cascade simulations both qualitatively and quantitatively. Even if a sufficiently accurate potential can be identified, the results represent a certain limiting case of what may be observed experimentally. This is because all the simulations mentioned so far were carried out in perfect material - computer-pure material. Nowhere in nature can such perfect metal be found, particularly for iron, which is easily contaminated with minor interstitial impurities such as carbon. In this section, a few examples will be discussed to illustrate how reality may influence cascade damage production relative to the perfect material case. The examples include the influence of preexisting defects, free surfaces, and grain boundaries.
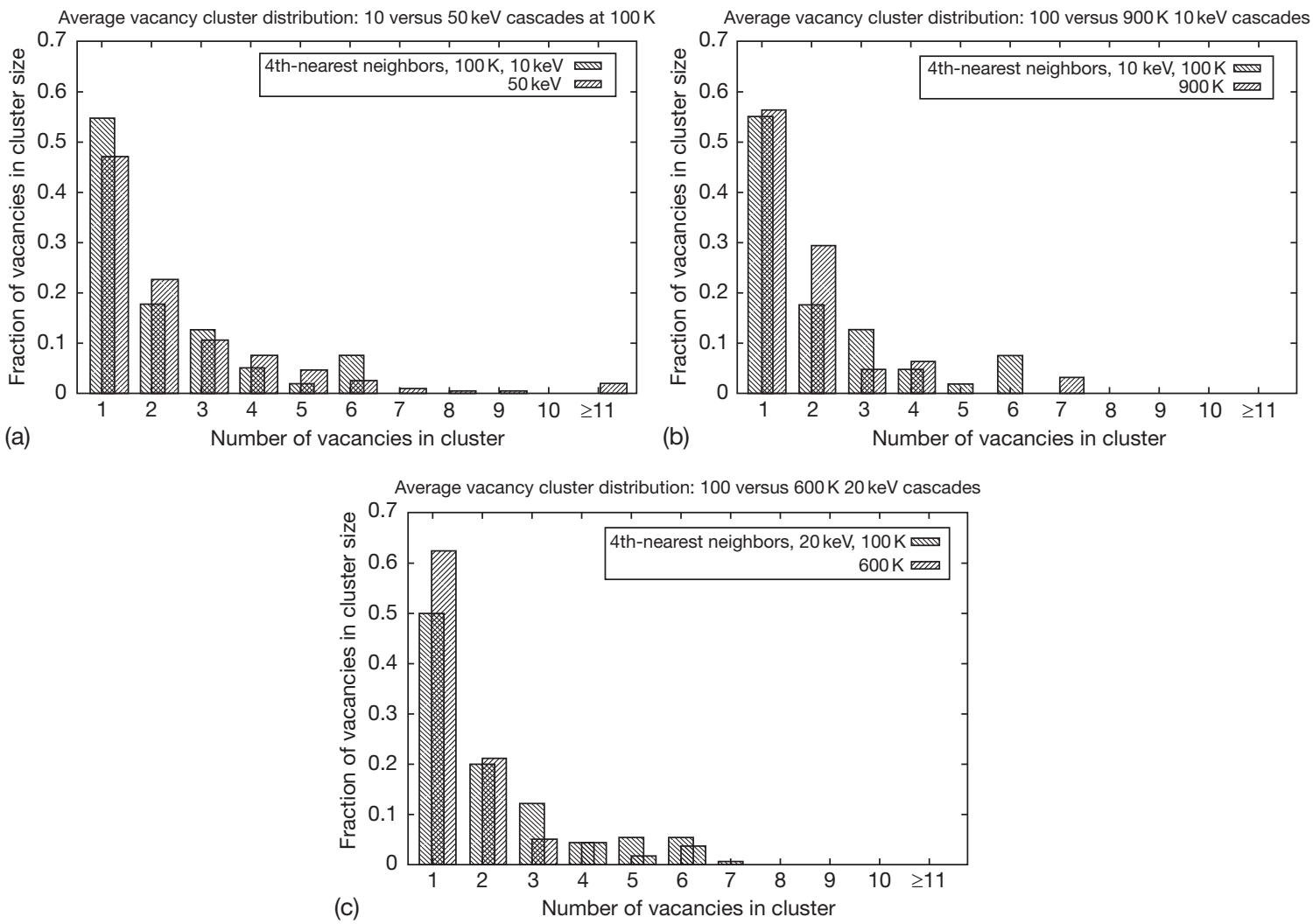

Figure 22 Fractional size distributions of loosely coupled vacancy clusters (all within fourth NN) formed directly within the cascade, comparison of (a) 10 and $50 \mathrm{keV}$ cascades at $100 \mathrm{~K}$, (b) $10 \mathrm{keV}$ cascades at 100 and $900 \mathrm{~K}$, and (c) $20 \mathrm{keV}$ cascades at 100 and $600 \mathrm{~K}$. 


\subsection{Influence of preexisting defects}

Even if a well-annealed, nearly defect-free, single crystal material is selected for irradiation, radiationinduced defects will rapidly change the state of the material. A simple calculation employing typical elastic scattering cross-sections for fast neutrons and the cascade volumes observed in MD simulations will demonstrate that by the time a dose of $\sim 0.01 \mathrm{dpa}$ is reached, essentially the complete volume will have experienced at least one cascade. There have been relatively few studies on how cascade damage production may be different in material with defects. ${ }^{95-97}$ The results of cascade simulations reported in Stoller and Guiriec $^{97}$ were carried out at $10 \mathrm{keV}$ and $100 \mathrm{~K}$ to expand the range of previous work carried out using $1 \mathrm{keV}$ simulations in copper ${ }^{95}$ and $0.40,2.0$, and $5.0 \mathrm{keV}$ simulations in iron. ${ }^{96} \mathrm{~A} 10 \mathrm{keV}$ cascade energy is high enough to initiate in-cascade clustering, is near the plateau region of the defect survival curve, and involves a limited degree of subcascade formation. For these conditions, the database discussed above (see Figure 11) includes two independent sets of cascades, seven in a $128 \mathrm{k}$ atom cell and eight in a $250 \mathrm{k}$ atom cell that can be used to provide a basis of comparison. A cell size of $250 \mathrm{k}$ atoms was used for the cascade simulations with preexisting damage.

The study in Stoller and Guiriec ${ }^{97}$ involved three simple configurations of preexisting damage that were all derived from cascade debris. This is perhaps the simplest possible damage structure, a collection of point defects and point defect clusters. The first configuration was simply the as-quenched debris from a $10 \mathrm{keV}$ cascade in perfect crystal. A total of 30 vacancies and interstitials were present, including one di- and one 7-interstitial cluster. The second case was similar, but the point defects were reconfigured so that the 30 vacancies included a 6-vacancy void and a 9-vacancy loop, and the interstitial clusters included four di-, one tri-, and one 8-interstitial cluster. The third configuration contained only a single 30-vacancy void. These configurations are shown in Figure 23. Eight simulations were carried out with different initial PKAs and the same $<135>$ PKA direction. The selected PKA were $\sim 15-20$ lattice parameters from the center of the cascade debris and located such that the $\langle 135\rangle$ direction pointed them toward the center of the debris field. The same set of PKAs was used for all three defect configurations.

As expected, substantial variation was observed between the different simulations for any given preexisting defect configuration; in some cases the cascade produced more defects than in perfect crystal,

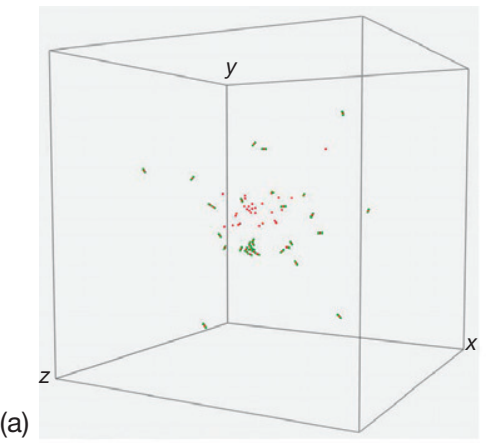

(a)

(b)

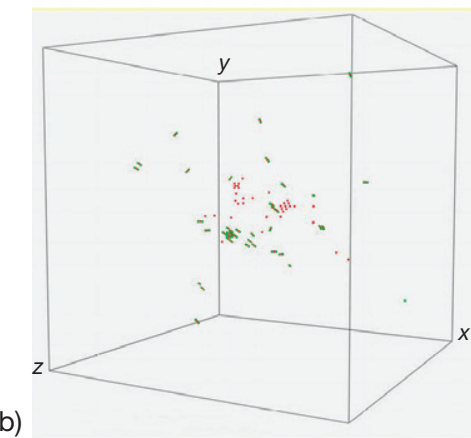

(c)

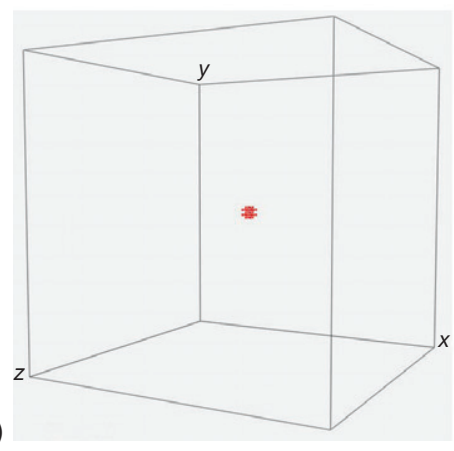

Figure 23 Initial defect distributions for investigation of effects of preexisting damage on defect formation in $10 \mathrm{keV}$ cascades: (a) $10 \mathrm{keV}$ cascade debris with $30 \mathrm{SIA}$ and 30 vacancies, (b) same number of defects as in (a) but clustering artificially increased, (c) 30-vacancy void.

while in others fewer were produced. The most dramatic visible effects were observed for the 30-vacancy void. In one case, the void was completely intact after the second cascade, while in the others it was destroyed to varying degrees. The impact of preexisting damage on stable defect formation in the $10 \mathrm{keV}$ cascades is shown in Figure 24, where results from the three different defect configurations are compared with those obtained in perfect crystal. The variation between two sets of perfect crystal simulations is provided for comparison purposes. The statistical information from analysis of defect survival 


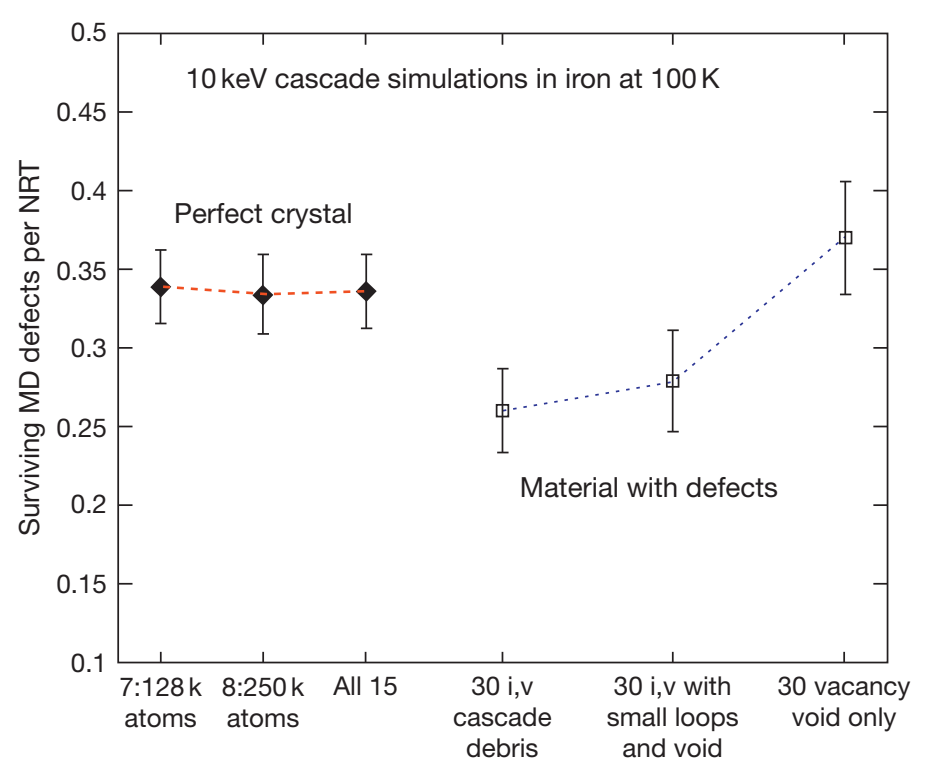

Figure 24 Comparison of defect survival values for cascades in perfect crystal and material with preexisting defects (i,v denotes interstitial and vacancy).

Table 3 Summary of defect production results from cascades with preexisting damage (i,v denotes interstitial and vacancy)

\begin{tabular}{|c|c|c|c|c|}
\hline & $\begin{array}{l}\text { Survival } \\
\text { fraction } \\
\text { (per NRT) }\end{array}$ & $\begin{array}{l}\text { Standard } \\
\text { error }\end{array}$ & $\begin{array}{l}\text { Interstitial cluster } \\
\text { fraction (per NRT) }\end{array}$ & $\begin{array}{l}\text { Standard } \\
\text { error }\end{array}$ \\
\hline $\begin{array}{l}\text { Perfect crystal (all } 15128 \mathrm{k} \text { and } 250 \mathrm{k} \text { atoms) } \\
\text { Defective crystal }\end{array}$ & 0.336 & 0.0137 & 0.170 & 0.0155 \\
\hline $\begin{array}{l}30 \text { i,v: cascade debris, with one di- and one } 7 \text {-interstitial } \\
\text { cluster }\end{array}$ & 0.260 & 0.0214 & 0.179 & 0.0119 \\
\hline $\begin{array}{l}30 \text { i,v: cascade debris with four di-, one tri-, and one } \\
\text { 8-interstitial cluster; 6-vacancy void, 9-vacancy loop }\end{array}$ & 0.279 & 0.0258 & 0.110 & 0.0191 \\
\hline 30-vacancy void only & 0.370 & 0.0288 & 0.190 & 0.0188 \\
\hline
\end{tabular}

and interstitial clustering is summarized in Table 3. On average, a significant reduction in defect formation was observed for the two configurations most typical of random cascade debris. A slight increase (that may not be statistically significant) in defect production was observed when the cell contained only a small void. Only the second defect configuration led to a significant change in interstitial clustering.

Although the approach in Stoller's investigation of preexisting damage was slightly different, the results are consistent with previous studies by Foreman and coworkers $^{95}$ and Gao and coworkers. ${ }^{96}$ They observed substantial reductions in defect production when a cascade was initiated in material containing defects. The reductions in defect production observed by Stoller (Figure 24 and Table 3) are somewhat smaller. This difference may partially be due to the higher cascade energy employed here $(10 \mathrm{keV}$ vs. 0.4 $5 \mathrm{keV}$ ), but the statistical nature of cascade evolution is also a factor. Gao and coworkers analyzed the results of several simulations as a function of distance between the center of mass (COM) of the new cascade and that of the preexisting damage. A good correlation was found between this spacing and the number of defects produced. In the work of Stoller and Guiriec, ${ }^{97}$ the distance between PKA location and the preexisting damage was nearly constant. As the morphology of each cascade is quite different, the COM spacings varied. This is certainly part of the reason for the variety of behaviors mentioned above for the case of the small void. The average behavior for a fixed initial separation cannot be 
directly compared to any of Gao's results for the average at a fixed distance. Many more simulations need to be carried out at different energies to develop a more complete picture of cascade damage formation in material with typical defect densities, particularly to assess the clustering behavior. Overall, the reduced defect survival observed in material containing defects suggests that it may be appropriate to employ defect formation values that are somewhat lower than the perfect crystal results in the kinetic models used to simulate microstructural evolution over long times.

\subsection{Influence of free surfaces}

The rationale for investigating the impact of free surfaces on cascade evolution is the existence of an influential body of experimental data provided by experiments in which thin foils are irradiated by high-energy electrons and/or heavy ions. ${ }^{98-106}$ In most cases, the experimental observations are carried out in situ by TEM and the results of MD simulations are in general agreement with the data from these experiments. For example, some material-to-material differences observed in the MD simulations, such as differences in in-cascade clustering between bcc iron and fcc copper, also appear in the experimental data. ${ }^{59,107,108}$ However, the yield of large point defect clusters in the simulations is lower than would be expected from the thin foil irradiations, particularly for vacancy clusters. It is desirable to investigate the source of this difference because of the influence this data has on our understanding of cascade damage formation.

Both simulations ${ }^{81,97,109,110}$ and experimental work $^{105,106}$ indicate that the presence of a nearby free surface can influence primary damage formation. For example, interesting effects of foil thickness have been observed in some experiments. ${ }^{105}$ Unlike cascades in bulk material, which produce vacancies and interstitials in equal numbers, the number of surviving vacancies in surface-influenced cascades can exceed the number of interstitials because of interstitial transport to the surface. This could lead to the formation of larger vacancy clusters and account for the differences in visible defect yield observed between the results of MD cascade simulations conducted in bulk material and the thin-film, in situ experiments. Initial modeling work reported by Nordlund and coworkers ${ }^{81}$ and Ghaly and Averback ${ }^{109}$ demonstrated the nature of effects that could occur, and Stoller and coworkers ${ }^{97,100}$ subsequently conducted a study involving a larger number of simulations at 10 and $20 \mathrm{keV}$ to determine the magnitude of the effects.

To carry out the simulations, ${ }^{97,100}$ a free surface was created on one face of a cubic simulation cell containing 250000 atom sites. Atoms with sufficient kinetic energy to be ejected from the free surface (sputtered) were frozen in place just above the surface. Periodic boundary conditions are otherwise imposed. Two sets of nine $100 \mathrm{~K}$ simulations at $10 \mathrm{keV}$ were carried out to evaluate the effect of the free surface on cascade evolution. In one case, all the PKAs selected were surface atoms and, in the other, PKA were chosen from the atom layer $10 a_{0}$ below the free surface. The PKA in eight $20 \mathrm{keV}, 100 \mathrm{~K}$ simulations were all surface atoms. Several PKA directions were used, with each of these directions slightly more than $10^{\circ}$ off the [001] surface normal.

Figure 25 provides a representative example of a cascade initiated at the free surface. The peak damage state at $\sim 1.1 \mathrm{ps}$ is shown in (a), with the final damage state at $\sim 15$ ps shown in (b). The large number of apparent vacancies and interstitials in
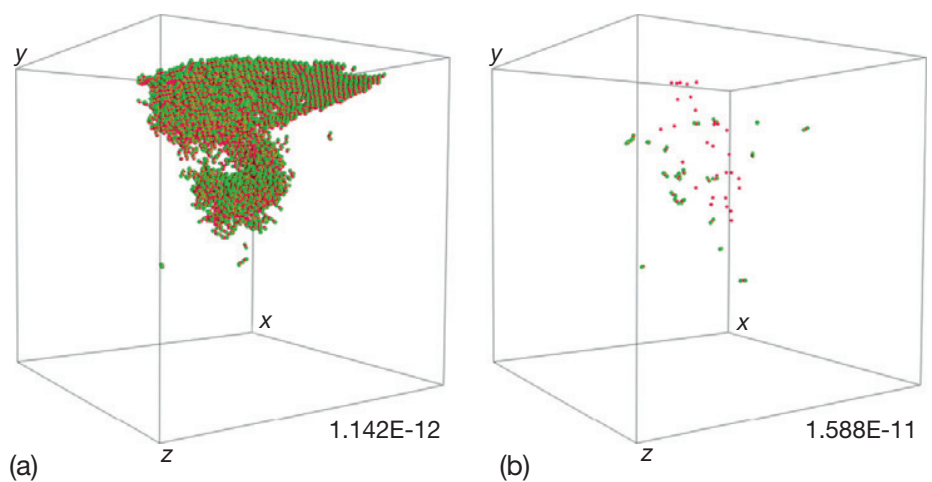

(b)

Figure 25 Defect evolution in typical $10 \mathrm{keV}$ cascade initiated by a surface atom: (a) peak damage state at $\sim 1.1 \mathrm{ps, \text {and }}$ (b) final damage state at $\sim 15 \mathrm{ps}$. 
Figure 25(a) is due to the pressure wave from the cascade reaching the free surface. With the constraining force of the missing atoms removed, this pressure wave is able to displace the near-surface atoms by more than $0.3 a_{0}$, which is the criterion used to choose atom locations to be displayed. As mentioned above, a similar pressure wave occurs in bulk cascades, making the maximum number of displaced atoms much greater than the final number of displacements. Most of these displacements are short-lived, as shown in Figure 26, in which the time dependence of the defect population is shown for three typical bulk cascades, one surface-initiated cascade, and one cascade initiated $10 a_{0}$ below the surface. The effect of the pressure wave persists longer in surface-influenced cascades, and may contribute to stable defect formation.

The number of surviving point defects (normalized to NRT displacements) is shown in Figure 27 for both bulk and surface cascades, with error bars indicating the standard error of the mean. The results are similar at 10 and $20 \mathrm{keV}$. Stable interstitial production in surface cascades is not significantly different than in bulk cascades; the mean value is slightly lower for the $10 \mathrm{keV}$ surface cascades and slightly higher for the $20 \mathrm{keV}$ case. However, there is a substantial increase in the number of stable vacancies produced, and the change is clearly significant. It is particularly worth noting that the number of surviving interstitials and vacancies is no longer equal for cascades initiated at the surface because interstitials can be lost by sputtering or the diffusion of interstitials and small glissile interstitial clusters to the surface. Reducing the number of interstitials leads to a greater number of surviving vacancies, as less recombination can occur.

In-cascade clustering of interstitials is also relatively unchanged in the surface cascades (e.g., see Figures 4 and 5 in Stoller ${ }^{110}$ ). The effect on incascade vacancy clustering was more substantial. The vacancy clustering fraction per NRT (based on the fourth $\mathrm{NN}$ criterion discussed above) increased from $\sim 0.15$ to 0.18 at $10 \mathrm{keV}$ and from $\sim 0.15$ to 0.25 at $20 \mathrm{keV}$. Moreover, the vacancy cluster size distributions changed dramatically, with larger clusters produced in the surface cascades. The free surface effect on the vacancy cluster size distributions obtained at $20 \mathrm{keV}$ bulk is shown in Figure 28. The largest vacancy cluster observed in the bulk cascades contained only six vacancies, while the surface cascades had clusters as large as 21 vacancies. This latter size is near the limit of visibility in TEM, with a diameter of almost $1.5 \mathrm{~nm}$. Overall, these results imply that cascade defect production in bulk material is different from that observed in situ using TEM. More research such as that by Calder and coworkers ${ }^{11}$ is required to fully assess these phenomena, particularly for higher cascade energies, in order to improve the ability to make quantitative comparisons between simulations and experiments.

\subsection{Influence of grain boundaries}

Depending on the complexity of the microstructure, internal interfaces such as grain boundaries, twins,

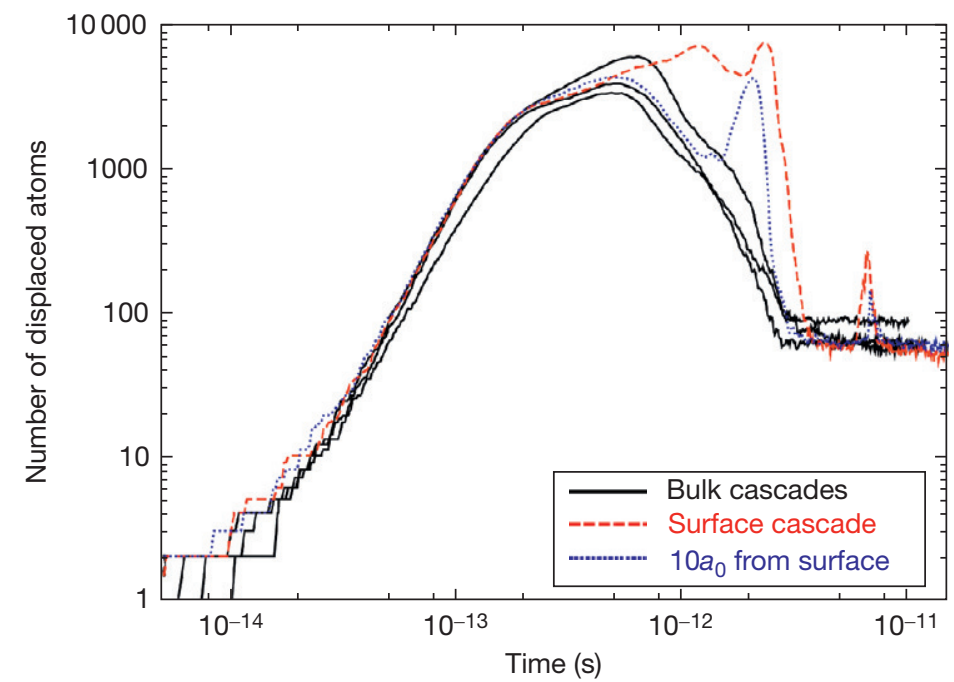

Figure 26 Time dependence of displaced atoms in $10 \mathrm{keV}$ cascades, three typical cascades initiated near the center of the cell are compared with a cascade initiated by an atom on a free surface and one initiated by an atom $10 a_{0}$ below the free surface. 

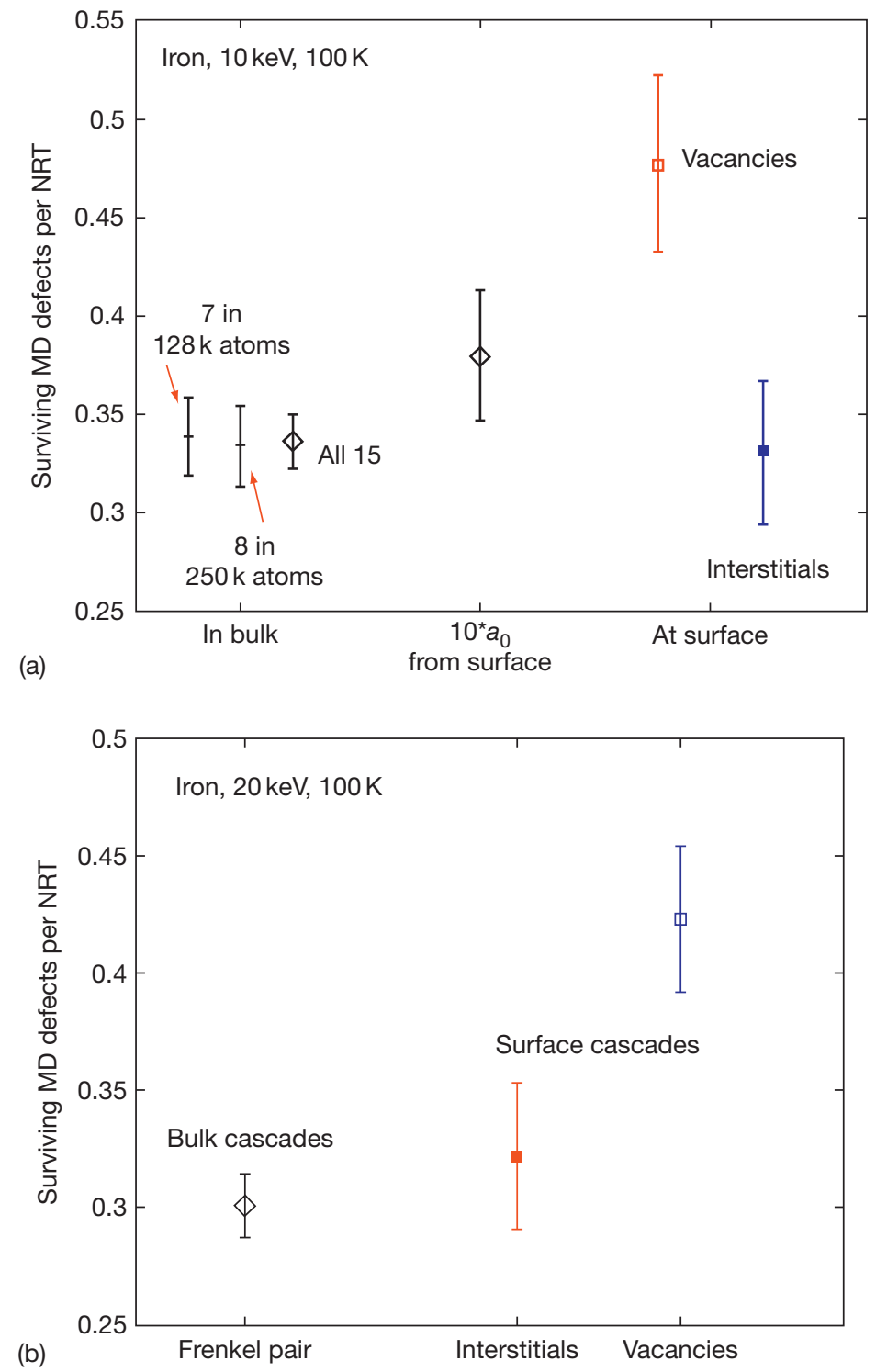

Figure 27 Average stable defect production in 10 and $20 \mathrm{keV}$ cascades. $10 \mathrm{keV}$ data compares two populations of bulk cascades, cascades initiated $10 a_{0}$ below the free surface, and cascades initiated at the free surface, $20 \mathrm{keV}$ cascades. $20 \mathrm{keV}$ results compare bulk and free surface cascades.

and lath and packet boundaries (in ferritic/martensitic steels) can provide a significant sink in the material for point defects. As such, they may play a significant role in radiation-induced microstructural evolution. For example, the effect of grain size on austenitic stainless steels was observed as early as $1972 .{ }^{112-114}$ The swelling effect was more closely associated with damage accumulation than damage production, but current understanding of the role of mobile interstitial clusters has provided a link to damage production as well (Singh and coworkers ${ }^{115}$ and Chapter
1.13, Radiation Damage Theory). More recently, there has been considerable interest in the properties of nanograined materials because the high sink strength could lead to very efficient point defect recombination and improved radiation resistance. It is reasonable to expect that primary damage production could be influenced in nanograined material because the grain sizes can be of comparable size to high-energy displacement cascades. Moreover, investigation of grain size effects by MD would be computationally limited to nanograin sizes in any case. 


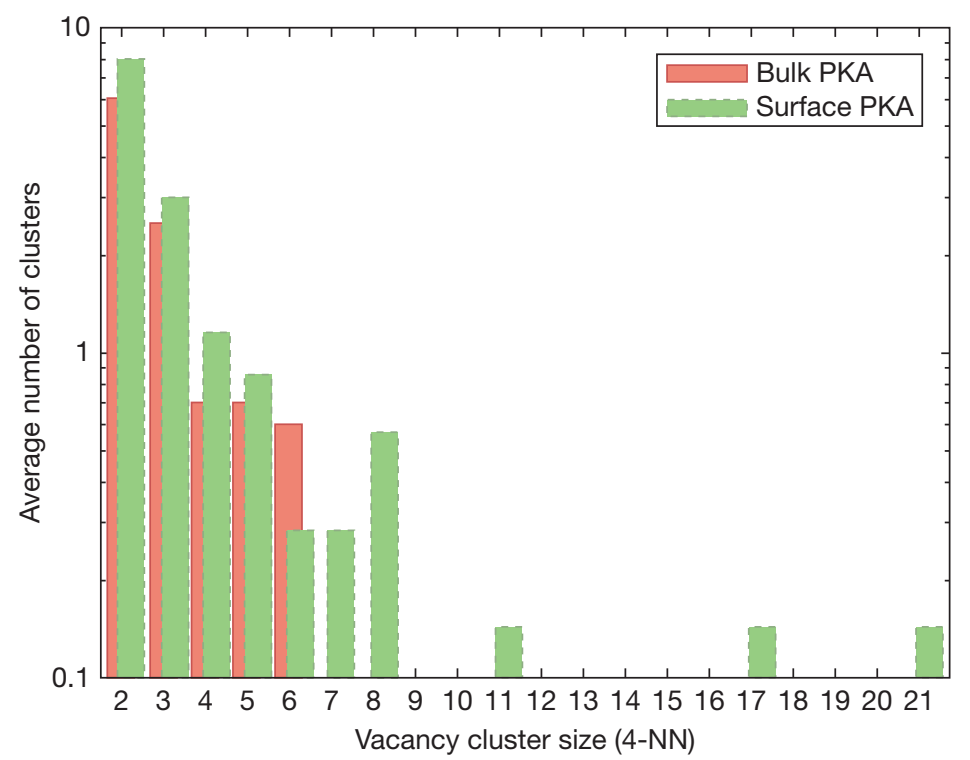

Figure 28 Comparison of in-cascade vacancy cluster size distribution in $20 \mathrm{keV}, 100 \mathrm{~K}$ cascades initiated by a PKA near the center (bulk) and at the free surface of the simulation cell.

To date, there have been a limited number of studies carried out to investigate whether and how primary damage formation would be altered in nanograined metals, ${ }^{116-121}$ and quite strong effects have been observed. ${ }^{116}$ The work from Stoller and coworkers ${ }^{122}$ will be used here to illustrate the phenomenon because the results of that study can be directly compared with the existing single crystal database that has been discussed above. A sufficient number of simulations were carried out at cascade energies of 10 and $20 \mathrm{keV}$ and temperatures of 100 and $600 \mathrm{~K}$ to obtain a statistically significant comparison. The results demonstrate that the creation of primary radiation damage can be substantially different in nanograined material due to the influence of nearby grain boundaries.

To create the nanocrystalline structure, grain nucleation sites were chosen, and the grains were filled using a Voronoi technique. ${ }^{123}$ A $2 \times 2 \times 2$ lattice parameter face-centered cubic (fcc) unit cell system was used to obtain the grain nucleation sites, resulting in 32 grains in the final sample. Each Voronoi polyhedron was filled with atoms placed on a regular bcc iron crystalline lattice, with the lattice orientation randomly selected. Grain boundaries occur naturally when the atomic plains in adjacent polyhedra impinge on one another, and overlapping atoms at the grain boundaries were removed. The final system was periodic and had an average grain size of $10 \mathrm{~nm}$, system box length of $28.3 \mathrm{~nm}$, and contained roughly 1.87 million atoms. More details on the procedure can

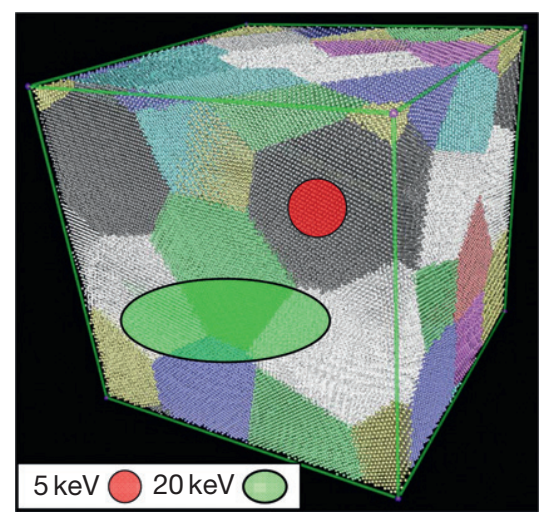

Figure 29 MD simulation cell, $32 \sim 10 \mathrm{~nm}$ grains. Shaded red circle and green ellipse indicate approximate size of 5 and $10 \mathrm{keV}$ cascades, respectively.

be found in Stoller and coworkers. ${ }^{122}$ The system was equilibrated for over $200 \mathrm{ps}$ including a heat treatment up to $600 \mathrm{~K}$. Figure 29 illustrates a typical grain structure with each grain shown in a different color. The approximate sizes of 5 and $20 \mathrm{keV}$ cascades have been projected on to the face of the simulation cell.

MD cascade simulations were carried out in the same manner discussed above, although the analysis was somewhat more difficult due to the need to differentiate cascade-produced defects from the defect structure associated with the grain boundaries. It was common for the cascade volume to cross from one grain to another. ${ }^{122}$ The number of vacancies and 
interstitials surviving in the nanograin simulations is compared to the single crystal results in Figure 30. A wider range of cascade energies is included in Figure 30(a) to show the trend in the single crystal data, while Figure 30(b) highlights the differences at the temperature and energy of the nanograin simulations. Mean values are indicated by the symbols in Figure 30(a) and the height of the bars in Figure 30(b), and the error bars indicate the standard error in both cases. Similar to the case for surfaceinfluenced cascades, the number of surviving vacancies and interstitials is not the same for cascades in nanograined material. The number of vacancies surviving in the nanograined material is similar to the single crystal data for $10 \mathrm{keV}$ cascades, but higher at
$20 \mathrm{keV}$. Much lower interstitial survival is observed in nanograined material under all conditions.

Consistent with the overall reduction in interstitial survival shown in Figure 30(a), the number of interstitials in clusters is dramatically reduced in nanograined material for all the conditions examined. As the number of surviving point defects, particularly interstitials, is so strongly reduced in the nanograin material, it is helpful to compare the fraction of defects in clusters in addition to the absolute number. Such a comparison is shown in Figure 31 where the fractions of surviving interstitials and vacancies contained in clusters in both nanograined and single crystal iron are compared for all the conditions simulated. The relative change in the clustering fraction is somewhat
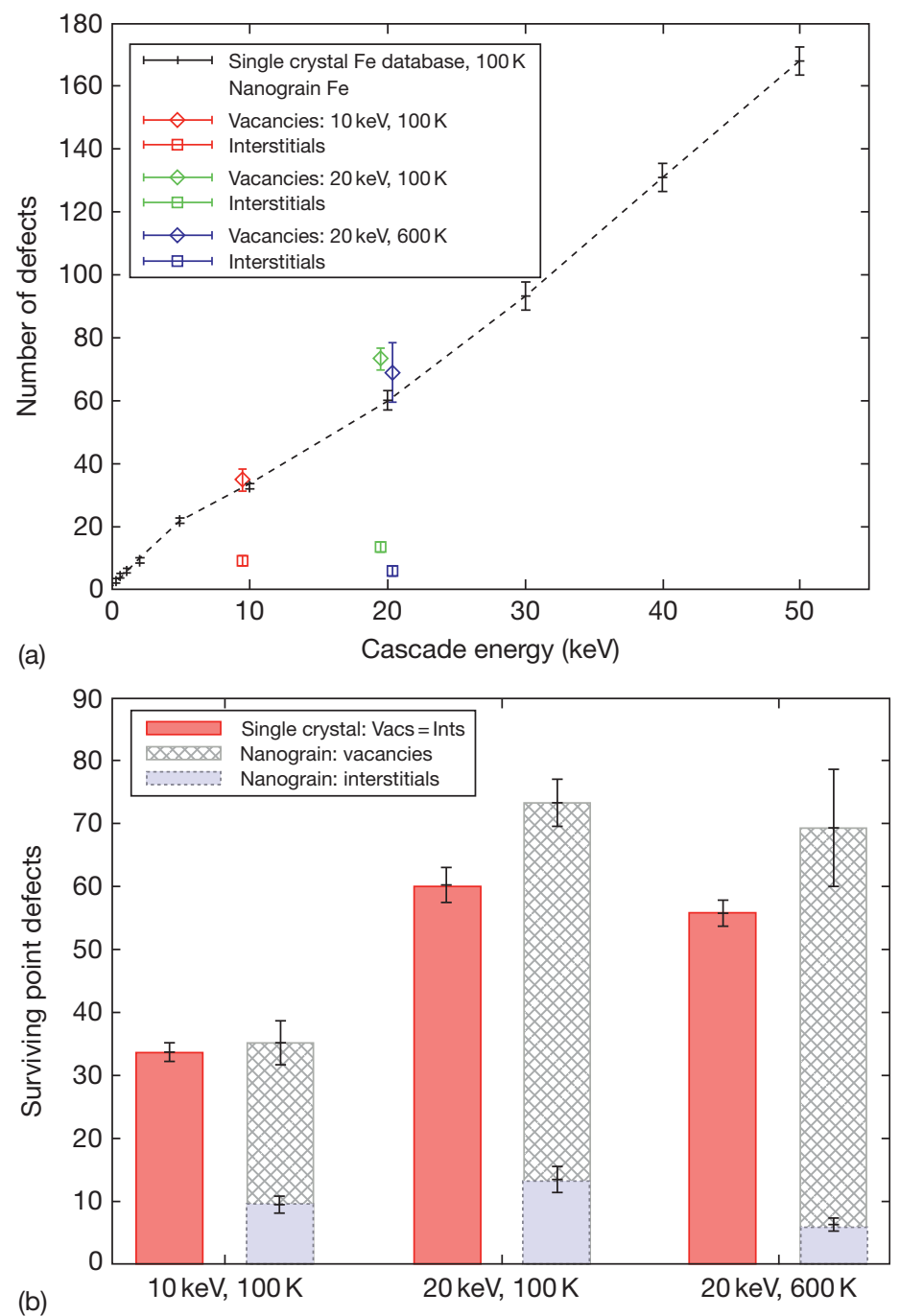

Figure 30 Number of stable interstitials and vacancies created by displacement cascades in single crystal and nanograined iron. 


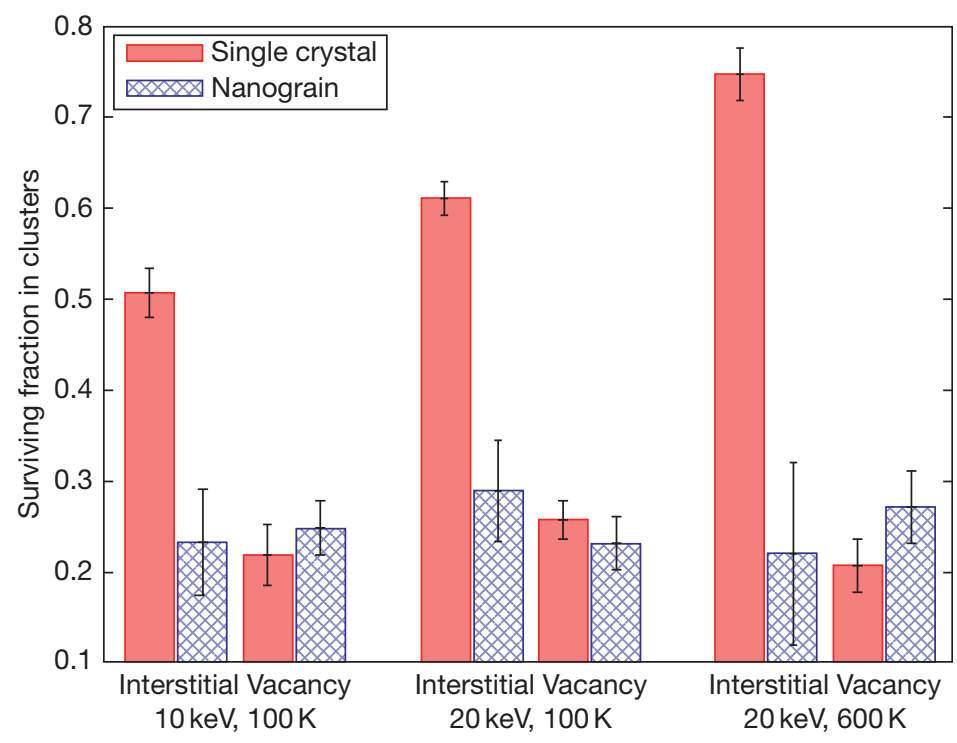

Figure 31 Fraction of surviving interstitials and vacancies contained in clusters in single crystal and nanograined iron.

less than the change in the total number of defects in clusters, but are still substantial for interstitial defects. Notably, the temperature dependence of clustering between 100 and $600 \mathrm{~K}$ observed in the single crystal $20 \mathrm{keV}$ cascades is reversed in nanograined material. Between 100 and $600 \mathrm{~K}$, the fraction of interstitials in clusters increases for single crystal iron but decreases for nanograined iron. Conversely, the vacancy cluster fraction decreases for single crystal iron and increases for nanograined iron.

Although the range of this study was limited in temperature and cascade energy, the results have demonstrated a strong influence of microstructural length scale (grain size) on primary radiation damage production in iron. Both the effects and the mechanisms appear to be consistent with previous work in nickel, ${ }^{16,120}$ in which very efficient transport of interstitial defects to the grain boundaries was observed. In both iron and nickel, this leads to an asymmetry in point defect survival. Many more vacancies than interstitials survive at the end of the cascade event in nanograined material while equal numbers of these two types of point defects survive in single grain material. Similar to single crystal iron, ${ }^{59,64}$ few of the vacancies have collapsed into compact clusters on the MD timescale. The vacancy clusters in both single and nanograined iron tend to be loose 3D aggregates of vacancies bound at the first and second NN distances as shown above in Figure 20. The size distribution of such vacancy clusters was not significantly different between the single and nanograin material. In contrast, the interstitial cluster size distribution was altered in the nanograined iron, with the number of large clusters substantially reduced. There appears to be both a reduction in the number of large interstitial clusters formed directly in the cascade and less coalescence of small mobile interstitial clusters as the latter are being transported to the grain boundaries.

The changes in defect survival observed in these simulations are qualitatively consistent with the limited available experimental observations. ${ }^{117-119}$ For example, Rose and coworkers ${ }^{117}$ carried out roomtemperature ion irradiation experiments of $\mathrm{Pd}$ and $\mathrm{ZrO}_{2}$ with grain sizes in the range of $10-300 \mathrm{~nm}$, and observed a systematic reduction in the number of visible defects produced. Chimi and coworkers ${ }^{118}$ measured the resistivity of ion irradiated gold specimens following ion irradiation and found that resistivity changes were lower in nanograined material after room-temperature irradiation. However, they observed an increased change in nanograined material following irradiation at $15 \mathrm{~K}$. The low-temperature results could be related to the accumulation of excess vacancy defects as they would be immobile at $15 \mathrm{~K}$.

\subsubsection{Comparison of Cascade Damage in Other Metals}

Differences in cascade damage formation between different metals was among the topics discussed at a workshop in 1998 entitled 'Basic Aspects of Differences in Irradiation Effects Between fcc, bcc, and hcp 
Metals and Alloys. ${ }^{124}$ The papers collected in that volume of the Journal of Nuclear Materials can be consulted to obtain the details on both damage production and damage evolution. A brief summary of the observed differences and similarities will be presented in this section. Although the development of alloy potentials is relatively recent, there have been a sufficient number of investigations to provide a comparison of displacement cascade evolution in pure iron with that in three binary alloys, $\mathrm{Fe}-\mathrm{C}, \mathrm{Fe}-\mathrm{Cu}$, and $\mathrm{Fe}-\mathrm{Cr} .{ }^{125-138}$ The motivation for each of these binary systems is clear. Carbon must be added to iron to make steel, and as a small interstitial solute it could interact with and influence interstitial-type defects. Copper is of interest largely because it is a primary contributor to reactor pressure vessel embrittlement when it is present as an impurity in concentrations greater than about 0.05 atom\% (Chapter 4.05, Radiation Damage of Reactor Pressure Vessel Steels). Steels containing $7-12$ atom $\%$ chromium are the basis of a number of modern ferritic and ferriticmartensitic steels that are of interest to nuclear energy systems (see Klueh and Harries ${ }^{133}$ ).

\subsubsection{Defect Production in Pure Metals}

As mentioned above in Section 1.11.4.2, Bacon and coworkers ${ }^{84,107}$ have shown that the number of stable point defects produced in many materials follows a simple power-law dependence over a broad range of cascade energies (see eqn [3]). This behavior is shown in Figure 32 for several pure metals and $\mathrm{Ni}_{3} \mathrm{Al}^{107}$ This figure also includes a line labeled NRT that is obtained from eqn [2] if the displacement threshold is taken as $40 \mathrm{eV}$, which is the recommended average value for iron. ${ }^{16}$ The difference between the NRT and Fe lines reflect the ratio plotted in Figure 9. As the displacement threshold is different for different metals (e.g., $30 \mathrm{eV}$ is recommended for $\mathrm{Cu}^{16}$ ), the other lines should not be compared directly with the NRT values. When normalized using the appropriate NRT displacements, the difference in the survival ratio between $\mathrm{Fe}$ and $\mathrm{Cu}$ can be seen in Figure $33 .{ }^{61}$ Although the stable defect production in the other metals may be either somewhat lower or higher than in iron, the behavior is clearly similar across this group of bcc, fcc, and hcp materials. As the energies involved in displacement cascades are so much greater than the energy per atom in a perfect lattice or the vacancy and interstitial formation energies, it is not surprising that ballistic defect production would be similar.

In-cascade clustering behavior shows a stronger variation between metals than does total defect survival. The fraction of surviving interstitials contained in clusters is shown in Figure $\mathbf{3 4}$ for some of these

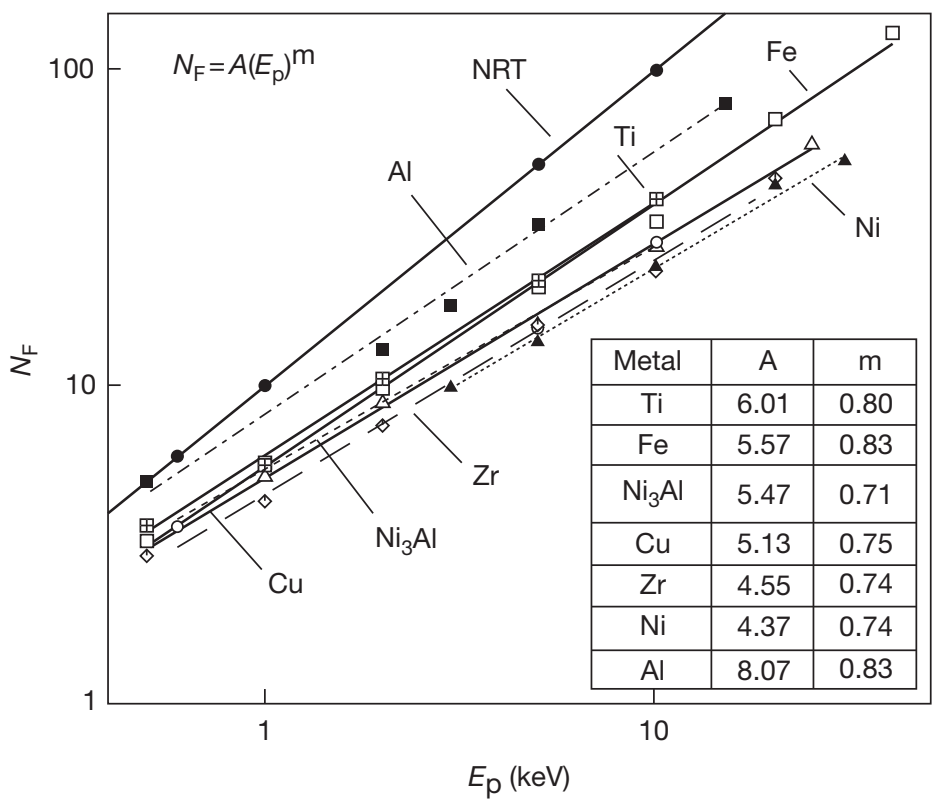

Figure 32 Stable defect formation as a function of cascade energy for several pure metals and $\mathrm{Ni}_{3} \mathrm{Al} 100 \mathrm{~K}$, and for $\mathrm{Al}$ and $\mathrm{Ni}$ at $10 \mathrm{~K}$. The inset table shows the values of $m$ and $A$ (with $E_{\mathrm{p}}$ in $\mathrm{keV}$ ) yielding the best power-law fit to the data. Reproduced from Bacon, D. J.; Gao, F.; Osetsky, Yu. N. J. Nucl. Mater. 2000, 276, 1-12. 


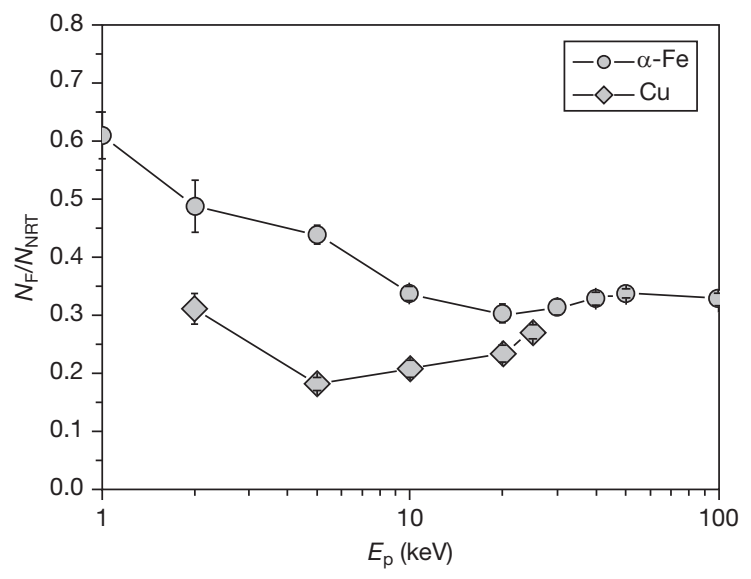

Figure 33 Total surviving Frenkel pair divided by the corresponding number of NRT displacements for $\mathrm{Fe}$ and $\mathrm{Cu} .{ }^{61}$ Displacement thresholds of $40 \mathrm{eV}$ and $30 \mathrm{eV}$ were used for $\mathrm{Fe}$ and $\mathrm{Cu}$, respectively. ${ }^{16}$

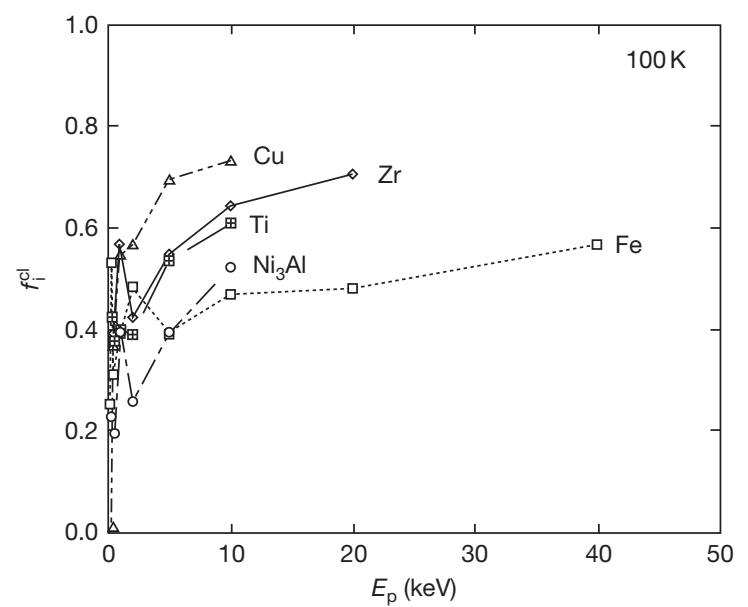

Figure 34 The fraction of surviving interstitials in clusters of two or more as a function of cascade energy at $100 \mathrm{~K}$ for $\mathrm{Cu}, \alpha-\mathrm{Fe}, \alpha-\mathrm{Ti}, \alpha-\mathrm{Zr}$, and $\mathrm{Ni}_{3} \mathrm{Al}$ at $100 \mathrm{~K}$. Reproduced from Bacon, D. J.; Gao, F.; Osetsky, Yu. N. J. Nucl. Mater. 2000, 276, 1-12.

same metals as in Figure 32. ${ }^{107}$ Although defect formation does not seem to correlate with crystal structure in Figure 32, there is some indication that this may not be the case with interstitial clustering. The lowest clustering fraction is seen in bcc Fe, while the close-packed $\mathrm{Cu}(\mathrm{fcc})$ and hcp (Ti, Zr) materials yield higher values. Ti and $\mathrm{Zr}$ exhibit nearly the same value. $\mathrm{Ni}_{3} \mathrm{Al}$, which is nominally close-packed is more similar to iron. This may be a result of the ordered structure and some impact of antisite defects on interstitial clustering. However, there is insufficient data available to make any definitive conclusions.
A further comparison of vacancy and interstitial clustering in $\mathrm{Fe}$ and $\mathrm{Cu}$ is provided by Figure $35,{ }^{61}$ which provides histograms of the cluster size distributions for two different cascade energies at $100 \mathrm{~K}$. The interstitial cluster size distributions are shown in (a) and (c) for $\mathrm{Fe}$ and $\mathrm{Cu}$, respectively, and the corresponding vacancy cluster size distributions are shown in (b) and (d). Note the scale difference on the abscissa between Figure 35( $\mathbf{a}$ and $\mathbf{b}$ ) and Figure 35(c and d). In addition to having a higher fraction of surviving defects in clusters, copper clearly produces much larger clusters of both types. It is clear that some of these differences are related to either the crystal structure and/or such basic parameters as the stacking fault energy. Many of the large vacancy clusters in fcc copper, which has a low stacking fault energy, are large stacking fault tetrahedra (generally imperfect). Similarly, large faulted SIA loops are observed in $\mathrm{Cu}$. Figure 36 illustrates the difference observed between iron and copper in typical $20 \mathrm{keV}$ cascades at $100 \mathrm{~K}$. The final damage state is shown for $\mathrm{Fe}$ in (a) and $\mathrm{Cu}$ in (b). The simulation cells have an edge length of 50 lattice parameters in both cases. The copper cascade is clearly more compact and exhibits more point defect clustering.

While comparisons of iron and copper have been thoroughly explored in the literature, ${ }^{59,61,107,139}$ there have also been studies on materials such as zirconium, which is relevant to nuclear fuel cladding..$^{70,107,140,141}$ Figure 37 provides an example of the differences in point defect clustering between Fe and hcp Zr. The average number of SIAs and vacancies in clusters per cascade as a function of cascade energy at $100 \mathrm{~K}$ is shown for (a) zirconium and (b) iron. ${ }^{107}$ Note the difference in scale on both the number in clusters and the cluster size, and that the highest cascade energy is $20 \mathrm{keV}$ in (a) and $49 \mathrm{keV}$ in (b). In both metals the probability of clustering increases with cascade energy, and the size of the largest cluster similarly increases. As indicated by the fact that there are more single vacancies than single interstitials, a greater fraction of SIAs are in clusters. Similar to the $\mathrm{Fe}-\mathrm{Cu}$ comparison, there is significantly more clustering in close-packed $\mathrm{Zr}$ than in bcc Fe.

\subsubsection{Defect Production in Fe-C}

Calder and coworkers examined the effect of carbon on defect production in the $\mathrm{Fe}-\mathrm{C}$ system with the carbon concentration between 0 and 1.0 atom $\%{ }^{125}$ The Fe potential was developed by Ackland and coworkers. ${ }^{134}$ The form of this potential is similar to 

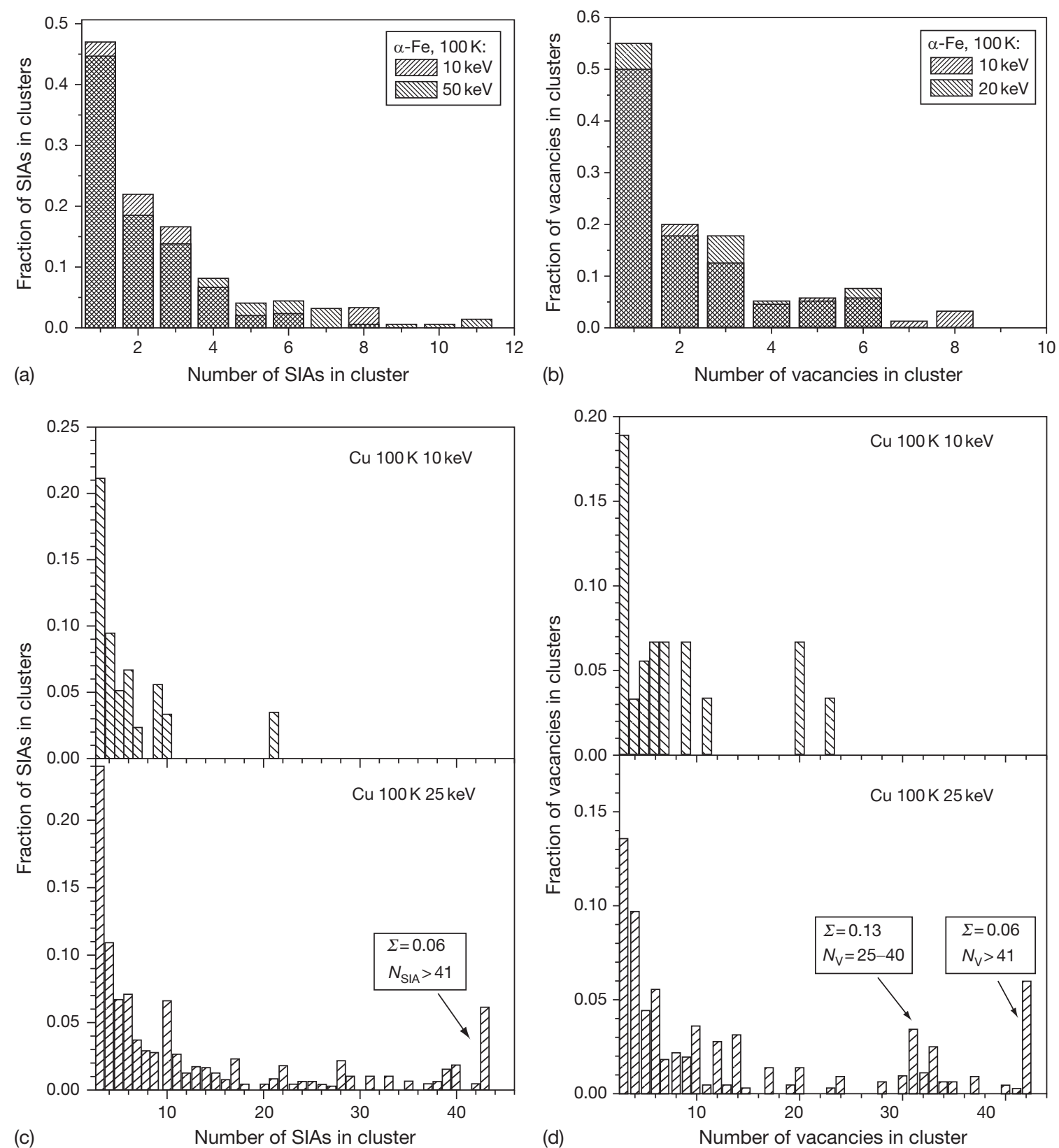

(c)

$$
\text { Number of SIAs in cluster }
$$

Figure 35 Comparison of in-cascade interstitial (a,c) and vacancy (b,d) cluster size distributions at $100 \mathrm{~K}$ for Fe (a,b) and Cu (c,d). Reproduced from Bacon, D. J.; Osetsky, Yu. N.; Stoller, R. E.; Voskoboinikov, R. E. J. Nucl. Mater. 2003, 323, $152-162$.

the Finnis-Sinclair potential discussed throughout this chapter, but the absolute level of defect production is somewhat lower. Simulations were carried out at temperatures of 100 and $600 \mathrm{~K}$ for cascade energies of 5,10 , and $20 \mathrm{keV}$. Thirty simulations were carried out at each condition to ensure a good statistical sampling. No systematic effect of carbon was observed on either stable defect formation or the clustering of vacancies and interstitials. Analysis of the octahedral sites around vacancies and interstitials revealed a statistically significant association of carbon atoms with both vacancies and SIAs. This indicates an effective trapping, which is consistent with the solute-defect binding energies. Although primary damage formation was not affected by carbon, the trapping mechanism could have an effect on damage accumulation. 


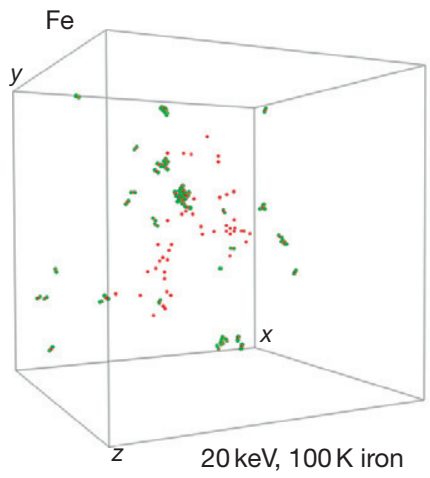

(a)

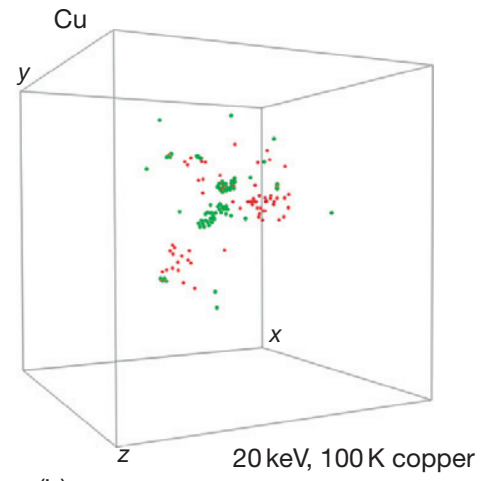

(b)

Figure 36 Comparison of stable defect production from a $20 \mathrm{keV}$ cascade at $100 \mathrm{~K}$ in $\mathrm{Fe}$ (a) and $\mathrm{Cu}$ (b). Note larger SIA clusters in (b).
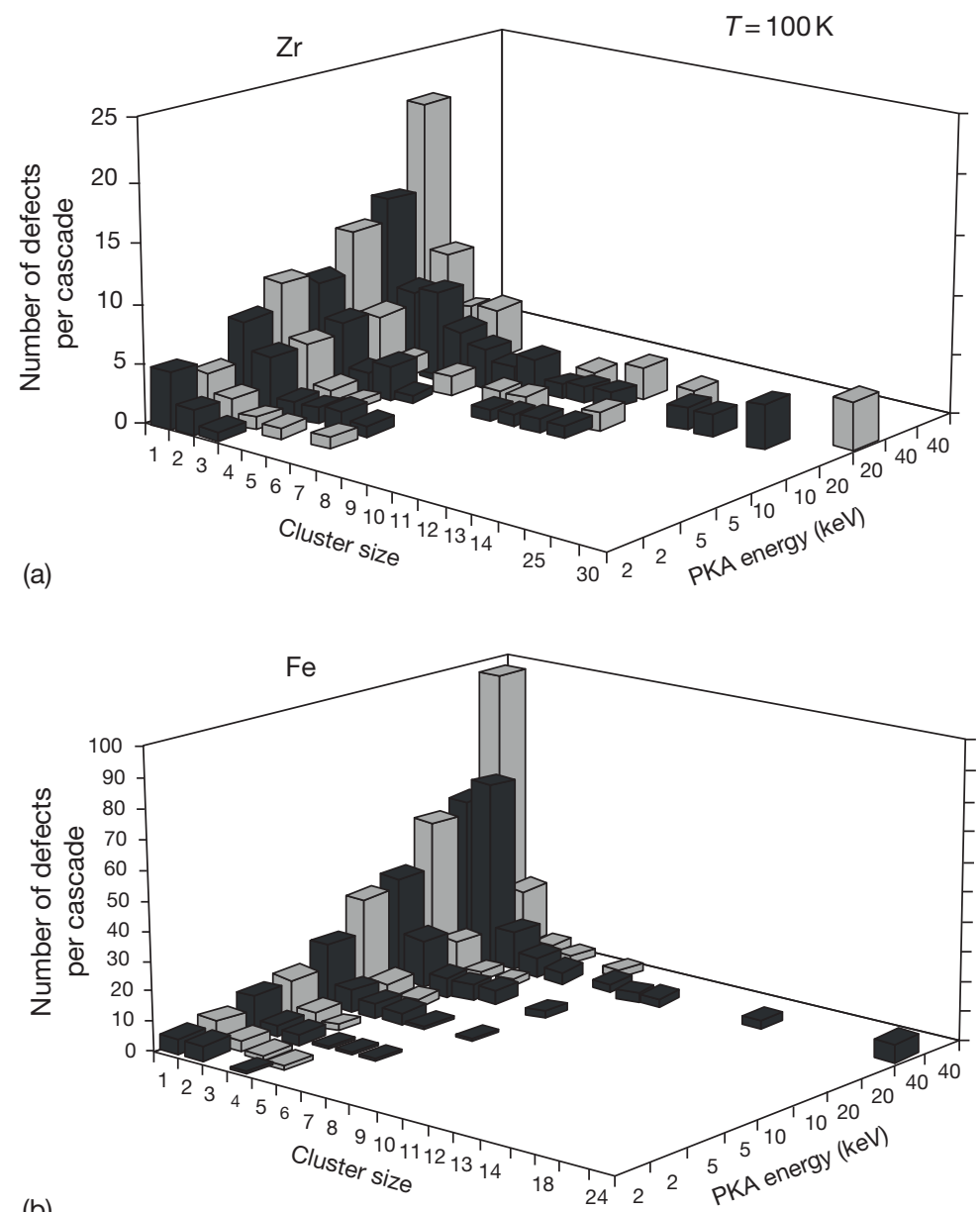

(b)

\section{Vacancy Interstitial}

Figure 37 The number of SIAs and vacancies in clusters per cascade as a function of cascade energy in (a) a-zirconium and (b) a-iron at $100 \mathrm{~K}$. The values were obtained by averaging over all cascades at each energy. Reproduced from Bacon, D. J.; Gao, F.; Osetsky, Yu. N. J. Nucl. Mater. 2000, 276, 1-12. 


\subsubsection{Defect Production in $\mathrm{Fe}-\mathrm{Cu}$}

Copper concentrations as high as $\sim 0.4$ atom $\%$ were found in early reactor pressure steels, largely due to both steel recycling and the use of copper as a corrosion-resistant coating on steel welding rods. Research that began in the 1970s demonstrated that this minor impurity was responsible for a significant fraction of the observed vessel embrittlement due to its segregation into a high density of very small (a few nanometer diameter) copper-rich solute clusters (Becquart and coworkers, ${ }^{126}$ Chapter 4.05, Radiation Damage of Reactor Pressure Vessel Steels). Becquart and coworkers employed MD cascade simulations to determine whether displacement cascades could play a role in the $\mathrm{Cu}$-segregation process, for example, by coalescing with vacancies in the cascade core during the cooling phase. The set of interatomic potentials used is described in Becquart and coworkers. ${ }^{126}$ Cascade energies of 5, 10, and $20 \mathrm{keV}$ were employed in simulations at $600 \mathrm{~K}$, with copper concentrations of $0,0.2$, and 2.0 atom $\%$. Similar to the case for $\mathrm{Fe}-\mathrm{C}$, no effect of copper was found on either stable defect formation or point defect clustering. The tendency for copper to be found bound with either a vacancy or an interstitial in solute-defect complex was observed. The copper-vacancy complexes may play a role in the formation of copperrich clusters over longer times, but no evidence for copper clustering was observed in the cascade debris. Similar results were found in an earlier study by Calder and Bacon. ${ }^{127}$ Overall, the results of the $\mathrm{Fe}-\mathrm{Cu}$ studies completed to date are consistent with the fact that $\mathrm{Fe}$ and $\mathrm{Cu}$ have similar masses and do not strongly interact.

\subsubsection{Defect Production in $\mathrm{Fe}-\mathrm{Cr}$}

Interest in ferritic and ferritic-martensitic steels has stimulated the development of $\mathrm{Fe}-\mathrm{Cr}$ potentials such as those discussed by Malerba and coworkers. ${ }^{129}$ These potentials have been applied to investigate the influence of $\mathrm{Cr}$ on displacement cascades ${ }^{130,131}$ and on point defect diffusion. ${ }^{132}$ The MD cascade study by Malerba and coworkers ${ }^{130}$ involved cascade energies from 0.5 to $15 \mathrm{keV}$ at $300 \mathrm{~K}$. In contrast to the $\mathrm{Fe}-\mathrm{C}$ and $\mathrm{Fe}-\mathrm{Cu}$ results discussed above, a slight increase in stable defect formation was observed in $\mathrm{Fe}-10 \% \mathrm{Cr}$ relative to pure $\mathrm{Fe}$. The asymptotic value of the defect survival ratio (relative to the NRT) at the highest energies was 0.28 for $\mathrm{Fe}$ and 0.31 for $\mathrm{Fe}-10 \% \mathrm{Cr}$. In a later study by the same authors, which involved a larger number of simulations and energies up to $40 \mathrm{keV}$, they also concluded that the presence of $10 \% \mathrm{Cr}$ did not lead to a change in the collisional phase of the cascade but rather reduced the amount of recombination during the cooling phase. ${ }^{131}$ Additional detailed studies performed with more recent $\mathrm{Fe}-\mathrm{Cr}$ potentials essentially confirmed the absence of any significant effect of $\mathrm{Cr}$ on primary damage in $\mathrm{Fe}-\mathrm{Cr}$ alloys as compared to pure Fe. $^{136-138}$

The lack of a $\mathrm{Cr}$ effect on the collisional or ballistic phase of the cascade may be expected because, like $\mathrm{Cu}$, the mass of $\mathrm{Cr}$ is similar to $\mathrm{Fe}$. The reduced recombination appears to be related to the formation of highly stable mixed $\mathrm{Fe}-\mathrm{Cr}$ dumbbell interstitials. About $60 \%$ of interstitial dumbbells contain a $\mathrm{Cr}$ atom, which is substantially higher than the overall $\mathrm{Cr}$ concentration of $10 \%$. In spite of the strong mixed dumbbell formation, the fraction of point defects in clusters did not seem to be significantly different than in pure Fe. However, if the stability and mobility of the mixed dumbbells and clusters containing them proves to be appreciably different than pure iron dumbbells, ${ }^{132}$ there could be an influence on damage accumulation at longer times. Experimental results that are consistent with this hypothesis ${ }^{135}$ are mentioned in Terentyev and coworkers. ${ }^{138}$

\subsubsection{Summary and Needs for Further Work}

The use of MD to simulate primary damage formation has become widespread and relatively mature. In addition to the research involving metals discussed above, the approach has also been applied to common structural ceramics ${ }^{11-14,142}$ and ceramics of interest to the nuclear fuel cycle. ${ }^{15,143,144}$ However, there are a number of areas that require further research. Some of these have to do with the most basic aspect of MD simulations, that is, the interatomic potentials that are used. In addition to the Finnis-Sinclair potential for iron that was used as a reference case in this chapter, results from several other iron potentials were mentioned. The choice of potential is never an obvious one, and there have been few studies to systematically compare them. In one of the studies mentioned in Section 1.11.3, the details of how one joins the equilibrium part of the potential to a screened Coulomb potential to account for shortrange interactions were shown to significantly 
influence cascade evolution and defect formation. ${ }^{41}$ Although a clear difference has been demonstrated, there is no clear path to determining what constitutes the 'correct' way to join these potentials.

In the case of iron and other magnetic elements such as chromium, research to address the issue of how magnetism may influence defect formation and behavior has only recently begun. ${ }^{145-147}$ The effect may be modest in the ballistic phase of the cascade when energies are high, but magnetism must certainly influence the configuration and properties of stable defects. Magnetic effects may also determine critical properties of interstitial clusters such as their migration energy and primary diffusion mechanism, which will strongly influence the nature of radiation damage accumulation. As the standard density functional theory fails to fully account for magnetic effects, further developments in electronic structure theory are required in order to provide data for fitting new and more accurate potentials.

The interaction between the atomic and electronic systems has largely been neglected in most of the work discussed above. This may impact the results of MD simulations in at least two ways. First, energetic atoms lose energy in a continuous slowing down process that involves both the elastic collisions MD currently models and electronic excitation and ionization between these elastic collisions with lattice atoms. Because of the energy dependence of elastic scattering cross-sections, neglecting the energy loss between atomic collisions could lead to more diffuse cascades and higher predicted defect survival. The second effect is related to inaccuracies in temperature when energy transfer between the electronic and atomic systems (electron-phonon coupling) is neglected. To first order, the atoms remain hotter when energy loss to the electron system is not accounted for. Given the temperature dependence of defect survival and defect clustering discussed above, this clearly has the potential to be significant in any one material. In addition, as electron-phonon coupling varies from one material to another, its neglect may obscure real differences in defect formation between materials.

Finally, the issue of rare events requires more investigation. The need to carry out sufficient simulations at a given condition to obtain an accurate estimate for mean behavior was emphasized in the chapter. However, it may be that rare events are also important for the prediction of radiation damage accumulation at longer times or higher doses. If nucleation of extended defects is difficult, which is typically the case at higher temperatures and lower point defect supersaturations, rare events that seed the microstructure with large clusters may largely control the process. One example of a potentially significant rare event is provided by the work of Soneda and coworkers. ${ }^{148}$ They carried out one hundred $50-\mathrm{keV}$ simulations at $600 \mathrm{~K}$ to obtain a good statistical description of defect formation at this condition. In one of these simulations, 223 stable point defects were created, which was much greater than the average of 130 defects. In addition, a $<100>$ vacancy loop containing 153 vacancies was created. The diameter of the loop was about $2.9 \mathrm{~nm}$, which is large enough to be visible by TEM. The impact of the one-in-a-hundred type events should not be underestimated without further study.

\section{Acknowledgments}

The author would like to acknowledge the fruitful collaboration and discussions on cascade damage for many years with Drs. David Bacon and Andrew Calder (University of Liverpool, UK), Lorenzo Malerba (SCK/CEN, Mol, Belgium), and Yuri Osetskiy (ORNL). He was first introduced to MD cascade simulations by Drs. Alan Foreman (deceased) and William Phythian during a short-term assignment at the AEA Technology Harwell Laboratory, UK, in the summer of 1994. Various aspects of the author's research discussed in this chapter were supported by the Division of Materials Sciences and Engineering, and the Office of Fusion Energy Sciences, US Department of Energy and the Office of Nuclear Regulatory Research of the US Nuclear Regulatory Commission at the Oak Ridge National Laboratory under contract DE-AC05-00OR22725 with UT-Battelle, LLC.

\section{References}

1. Garner, F. A. In Optimizing Materials for Nuclear Applications; Garner, F. A., Gelles, D. S., Wiffen, F. W., Eds.; The Metallurgical Society: Warrandale, PA, 1984; pp. 111-141.

2. Wang, J. A.; Kam, F. B. K.; Stallman, F. W. In Effects of Radiation on Materials; Gelles, D. S., Nanstad, R. K., Kumar, A. S., Little, E. A., Eds.; American Society of Testing and Materials: Philadelphia, PA, 1996; pp. 500-521, ASTM STP 1270.

3. Stoller, R. E. In Effects of Radiation on Materials; Gelles, D. S., Nanstad, R. K., Kumar, A. S., Little, E. A., Eds.; American Society for Testing and Materials: Philadelphia, PA, 1996; pp. 25-58, ASTM STP 1270. 
4. For example, see the proceedings from a series of two international conferences: (a) The Effects of Radiation on Materials, published in a series of Special Technical Publications by the ASTM International, West Conshohocken, PA; (b) the International Conference on Fusion Reactor Materials published in the Journal of Nuclear Materials.

5. Brinkman, J. A. J. Appl. Phys. 1954, 25, 961-969.

6. Brinkman, J. A. Am. J. Phys. 1956, 24, 246-267.

7. Tasch, A. F. Nucl. Inst. Meth. Phys. Res. 1993, 74B, 3-6.

8. Nicolet, M.-A.; Picraux, S. T. Eds.; Ion Mixing and Surface Layer Alloying, Noyes Publications: Park Ridge, NJ, 1984.

9. Mansur, L. K. J. Nucl. Mater. 2003, 318, 14-25.

10. Hobbs, L. W.; Pascucci, M. R. J. Phys. Colloques 1980, 41, C6-237-C6-242.

11. Uberuaga, B. P.; Voter, A. F.; Sickafus, K. E.; Cleave, A.; Grimes, R. W.; Smith, R. J. Comput. Aided Mater. Des. 2007, 14, 183-189.

12. Cleave, R.; Grimes, R. W.; Smith, R.; Uberuaga, B. P.; Sickafus, K. E. Nucl. Instr. Meth. B 2006, 250, 28-35.

13. Perlado, J. M.; Malerba, L.; Sanchez-Rubio, A.; Diaz de la Rubia, T. J. Nucl. Mater. 2000, 276, 235-242.

14. Gao, F.; Weber, W. J.; Devanathan, R. Nucl. Instr. Meth. B 2001, 180, 176-186.

15. Devanathan, R.; Weber, W. J. Nucl. Instr. Meth. B 2010, 268, 2857-2862.

16. ASTM E521, Standard Practice for Neutron Radiation Damage Simulation by Charged Particle Irradiation. Annual Book of ASTM Standards; ASTM International: West Conshohocken, PA; Vol. 12.02.

17. Kinchin, G. H.; Pease, R. S. Prog. Phys. 1955, 18, 1.

18. Lindhard, J.; Scharff, M.; Schiott, H. E. Mat. Fys. Medd. Dan. Vid. Selsk. 1963, 33, 1.

19. Norgett, M. J.; Robinson, M. T.; Torrens, I. M. Nucl. Eng. Des. 1975, 33, 50-54; see ASTM E693, Standard Practice for Characterizing Neutron Exposures in Ferritic Steels in Terms of Displacements per Atom (dpa). Annual Book of ASTM Standards; ASTM International: West Conshohocken, PA, Vol. 12.02.

20. Bacon, D. J.; Calder, A. F.; Harder, J. M.; Wooding, S. J. J. Nucl. Mater. 1993, 205, 52-58.

21. Finnis, M. W.; Sinclair, J. E. Phil. Mag. 1984, A50, 45-55; Erratum, Phil. Mag. 1986, A53, 161.

22. Nordlund, K.; Wallenius, J.; Malerba, L. Nucl. Instr. Meth. B 2006, 246, 322-332.

23. Torrens, I. M.; Robinson, M. T. In Radiation-Induced Voids in Metals; U.S. AEC CONF-710601; 1972, pp. 739-756.

24. Robinson, M. T. J. Nucl. Mater. 1994, 216, 1-28.

25. Stoller, R. E.; Odette, G. R. J. Nucl. Mater. 1992, 186, 203-205.

26. Jung, P. Phys. Rev. B 1981, 23, 664-670.

27. Wallner, G.; Anand, M. S.; Greenwood, L. R.; Kirk, M. A.; Mansel, W.; Waschkowski, W. J. Nucl. Mater. 1988, 152, 146-153.

28. Bergner, F.; Ulbrichta, A.; Hernandez-Mayoral, M.; Pranzas, P. K. J. Nucl. Mater. 2008, 374, 334-337.

29. Franz, H.; Wallner, G.; Peisl, J. Radiat. Eff. Def. Solids 1994, 128, 189.

30. Asoka-Kumar, P.; Lynns, K. G. J. Phys. IV 1995, 111(5), C1-C15.

31. Beaven, L. A.; Scanlan, R. M.; Seidman, D. N. The Defect Structure of Depleted Zones in Irradiated Tungsten; U.S. AEC Report NYO-3504-50; Cornell University: Ithaca, NY, 1971.

32. English, C. A.; Jenkins, M. L. Mater. Sci. Forum 1987, 15-18, 1003-1022.
33. Jenkins, M. L.; Kirk, M. A.; Phythian, W. J. J. Nucl. Mater 1993, 205, 16-30.

34. Kirk, M. A. In Microstructure of Irradiated Materials, Robertson, I. M., Rehn, L. E., Zinkle, S. J., Phythian, W. J., Eds.; Materials Research Society: Pittsburgh, PA, 1995; pp. 47-56.

35. Zinkle, S. J. and Singh, B. N. C

36. Kiritani, M. J. Nucl. Mater. 2000, 276, 41-49.

37. Gibson, J. B.; Goland, A. N.; Milgram, M.; Vineyard, G. H. Phys. Rev. 1960, 120, 1229.

38. Beeler, J. R., Jr. In Radiation-Induced Voids in Metals; U.S. AEC CONF-710601; 1972; pp. 684-738.

39. Heinisch, H. L.; Singh, B. N. J. Nucl. Mater. 1992, 191-194, 1083-1087.

40. Hou, M.; Souidi, A.; Becquart, C. S.; Domain, C.; Malerba, L. J. Nucl. Mater. 2008, 382, 103-111.

41. Malerba, L. J. Nucl. Mater. 2006, 351, 28-38.

42. Terentyev, D.; Lagerstedt, C.; Olsson, P.; et al. J. Nucl. Mater. 2006, 351, 65-77.

43. Stoller, R. E. J. Nucl. Mater. 1996, 233-237, 999-1003.

44. Stoller, R. E.; Guiriec, S. G. J. Nucl. Mater. 2004, 329-333, 1228-1232.

45. Stoller, R. E. In Microstructural Processes in Irradiated Materials; Lucas, G. E., Snead, L. L., Kirk, M. A., Elliman, R. G., Eds.; Materials Research Society: Warrandale, PA, 2001; Vol. 650, pp. R3.5.1-R3.5.6, MRS.

46. Mason, D. R.; le Page, J.; Race, C. P.; Foulkes, W. M. C.; Finnis, M. W.; Sutton, A. P. J. Phys. Condens. Matter. 2007, 19, 436209.

47. Finnis, M. W.; Agnew, P.; Foreman, A. J. E. Phys. Rev. B 1991, 44, 567-574.

48. Flynn, C. P.; Averback, R. S. Phys. Rev. B 1988, 38 7118-7120.

49. Caro, A.; Victoria, M. Phys. Rev. A 1989, 40, 2287-2291.

50. Gao, F.; Bacon, D. J.; Flewitt, P. E. J.; Lewis, T. A. Mod. Simul. Mater. Sci. Eng. 1998, 6, 543-556.

51. Race, C.P.; Mason, D. R.; Finnis, M. W.; Foulkes, W. M. C.; Horsfield, A. P.; Sutton, A. P. Rep. Prog. Phys. 2010, 73, 116501.

52. Averback, R. S.; Diaz de la Rubia, T.; Benedek, R. Nucl. Instr. Meth. Phys. Res. B 1988, 33, 693-700.

53. Diaz de la Rubia, T.; Guinan, M. W. J. Nucl. Mater. 1990, 274, 151-157.

54. Foreman, A. J. E.; English, C. A.; Phythian, W. J. Phil. Mag. 1992, 66A, 655-669.

55. Diaz de la Rubia, T.; Guinan, M. W. Mater. Sci. Forum 1992, 97-99, 23-42.

56. Averback, R. S. J. Nucl. Mater. 1994, 216, 49-62.

57. Adams, J. B.; Rockett, A.; Kieffer, J.; et al. J. Nucl. Mater. 1994, 216, 265-274.

58. Calder, A. F.; Bacon, D. J. J. Nucl. Mater. 1993, 207, 25-45.

59. Phythian, W. J.; Stoller, R. E.; Foreman, A. J. E.; Calder, A. F.; Bacon, D. J. J. Nucl. Mater. 1995, 223, 245-261.

60. Wooding, S. J.; Bacon, D. J.; Phythian, W. J. Phil. Mag. A 1995, 72, 1261-1279.

61. Bacon, D. J.; Osetsky, Yu. N.; Stoller, R. E.; Voskoboinikov, R. E. J. Nucl. Mater. 2003, 323, 152-162.

62. Nordlund, K.; Ghaly, M.; Averback, R. S.; Caturla, M.; Diaz de la Rubia, T.; Tarus, J. Phys. Rev. B 1998, 57, 7556-7570.

63. Stoller, R. E. In Microstructure of Irradiated Materials; Robertson, I. M., Rehn, L. E., Zinkle, S. J., Phythian, W. J., Eds.; Materials Research Society: Pittsburgh, PA, 1995; Vol. 373, pp. 21-26.

64. Stoller, R. E.; Odette, G. R.; Wirth, B. D. J. Nucl. Mater. 1997, 251, 49-60. 
65. Stoller, R. E. In Microstructural Processes in Irradiated Materials; Zinkle, S. J., Lucas, G. E., Ewing, R. C., Williams, J. S., Eds.; Materials Research Society: Pittsburgh, PA, 1999; Vol. 540, pp. 679-684.

66. Stoller, R. E. J. Nucl. Mater. 2000, 276, 22-32.

67. Stoller, R. E.; Calder, A. F. J. Nucl. Mater. 2000, 283-287, 746-752.

68. Caturla, M. J.; De la Rubia, T. D.; Victoria, M.; Corzine, R. K.; James, M. R.; Greene, G. A. J. Nucl. Mater. 2001 296, 90.

69. Fikar, J.; Schäublin, R. J. Nucl. Mater. 2009, 386-388, 97-101.

70. Wooding, S. J.; Bacon, D. J. Phil. Mag. A 1997, 76, 1033-1051.

71. Wirth, B. D.; Odette, G. R.; Maroudas, D.; Lucas, G. E. J. Nucl. Mater. 1997, 244, 185-194.

72. Becquart, C. S.; Domain, C.; Legris, A.; Van Duysen, J. C. J. Nucl. Mater. 2000, 280, 73-85.

73. Björkas, C.; Nordlund, K. NIMB 2007, 259, 853-860.

74. Malerba, L.; Marinica, M. C.; Anento, N.; et al. J. Nucl. Mater. 2010, 406, 19-38.

75. Finnis, M. W. MOLDY6 A Molecular Dynamics Program for Simulation of Pure Metals, AERE R 13182, U.K.A.E.A.; Harwell Laboratory, 1988.

76. Parrinello, M.; Rahman, A. Phys. Rev. Lett. 1980, 45, 1196-1199; Parrinello, M.; Rahman, A. J. Appl. Phys. 1981, 52, 7182.

77. Gao, F.; Bacon, D. J.; Flewitt, P. E. J.; Lewis, T. A. J. Nucl. Mater. 1997, 249, 77-86.

78. Stuart, A.; Ord, J. K. Kendall's Advanced Theory of Statistics; Charles Griffin and Company, Ltd.: London, 1987; Vol. 1.

79. Manual on Presentation of Data and Chart Control Analysis; American Society for Testing and Materials: West Conshohocken, PA, 1992; 6th edn.

80. Calder, A. F.; Bacon, D. J.; Barashev, A. V.; Osetsky, Yu. N. Phil. Mag. 2010, 90, 863-884.

81. Nordlund, K.; Keinonen, J.; Ghaly, M.; Averback, R. S. Nature 1999, 398, 49-51.

82. Stoller, R. E.; Greenwood, L. E. J. Nucl. Mater. 1999, 271-272, 57-62.

83. Oen, O. S.; Robinson, M. T. Appl. Phys. Lett. 1963, 2 , 83-85.

84. Bacon, D. J.; Calder, A. F.; Gao, F.; Kapinos, V. G.; Wooding, S. J. Nucl. Instr. Meth. Phys. Res. 1995, 102B, 37-46.

85. Averback, R. S.; Benedek, R.; Merkle, K. L. Phys. Rev. B 1978, 18, 4156-4171.

86. Gao, F.; Bacon, D. J.; Osetskiy, Yu. N.; Flewitt, P. E. J.; Lewis, T. A. J. Nucl. Mater. 2000, 276, 213-220.

87. Terentyev, D. A.; Klaver, T. P. C.; Olsson, P.; et al. Phys. Rev. Lett. 2008, 100, 145003.

88. Diaz de la Rubia, T.; Guinan, M. W. Phys. Rev. Lett. 1991, $66,2766$.

89. Calder, A. F.; Bacon, D. J.; Barashev, A. V.; Osetsky, Yu. N. Phil. Mag. Lett. 2008, 88, 43.

90. Wirth, B. D. Ph. D. Dissertation, University of California, Santa Barbara, 1998.

91. Djurabekova, F.; Malerba, L.; Pasianotcd, R. C.; Olssone, P.; Nordlund, K. Phil. Mag. 2010, 90, 2585-2595.

92. Sato, S.; Kohyama, A.; Igata, N. Appl. Surf. Sci. 1994, 76-77, 285-290.

93. Wirth, B. D.; Odette, G. R. In Proceedings of Symposium on Multiscale Modeling of Materials; Butalov, V. V., Diaz de la Rubia, T., Phillips, P., Kaxiras, E., Ghoniem, N., Eds.; Materials Research Society: Pittsburgh, PA, 1999; Vol. 527, pp. 211-216.
94. Valo, M.; Krause, R.; Saarinen, K.; Hautojarvi, P.; Hawthorne, J. R. In Effects of Radiation on Materials; Stoller, R. E., Kumar, A. S., Gelles, D. S., Eds.; American Society for Testing and Materials: West Conshohocken, PA, 1992; pp. 172-185, ASTM STP 1125.

95. Foreman, A. J. E.; Phythian, W. J.; English, C. A. private communication, unpublished.

96. Gao, F.; Bacon, D. J.; Calder, A. F.; Flewitt, P. E. J.; Lewis, T. A. J. Nucl. Mater. 1996, 276, 47-56.

97. Stoller, R. E.; Guiriec, S. G. J. Nucl. Mater. 2004, 329-333, 1228-1232.

98. Jenkins, M. L.; English, C. A.; Eyre, B. L. Phil. Mag. 1978, 38, 97.

99. Robertson, I. M.; Kirk, M. A.; King, W. E. Scripta Met. 1984, 18, 317-320.

100. English, C. A.; Jenkins, M. L. Mat. Sci. Forum 1987, 15-18, 1003-1022.

101. Kirk, M. A.; Robertson, I. M.; Jenkins, M. L.; English, C. A.; Black, T. J.; Vetrano, J. S. J. Nucl. Mater. 1987, 149, 21-28.

102. Vetrano, J. S.; Bench, M. W.; Robertson, I. M.; Kirk, M. A. Met. Trans. 1989, 20A, 2673-2680.

103. Jenkins, M. L.; Kirk, M. A.; Phythian, W. J. J. Nucl. Mater 1993, 205, 160.

104. Daulton, T. L.; Kirk, M. A.; Rehn, L. E. J. Nucl. Mater. 2000, 276, 258-268.

105. Kiritani, M. Mat. Sci. Forum 1987, 15-18, 1023-1046.

106. Muroga, T.; Yoshida, N.; Tsukuda, N.; Kitajima, K.; Eguchi, M. Mat. Sci. Forum 1987, 15-18, 1097-1092.

107. Bacon, D. J.; Gao, F.; Osetsky, Yu. N. J. Nucl. Mater. 2000, 276, 1-12.

108. Caturla, M. J.; Soneda, N.; Alonso, E.; Wirth, B. D.; Diaz de la Rubia, T.; Perlado, J. M. J. Nucl. Mater. 2000, 276, 13-21.

109. Ghaly, M.; Averback, R. S. Phys. Rev. Let. 1994, 72, 364.

110. Stoller, R. E. J. Nucl. Mater. 2002, 307-311, 935-940.

111. Calder, A. F.; Bacon, D. J.; Barashev, A. V.; Osetsky, Yu. N. Phil. Mag. 2008, 88, 43-53.

112. Leitnaker, J. M.; Bloom, E. E.; Stiegler, J. O. Fuels and Materials Development: Quarterly Progress Report; ORNL-TM-4105; Oak Ridge National Laboratory, 1972; pp. 3.19-3.29.

113. Horsewell, A.; Singh, B. N. In Effects of Radiation on Materials, Garner, F. A., Perrin, J. S., Eds.; American Society of Testing and Materials: Philadelphia, PA, 1985; pp. 248-261, ASTM STP 870.

114. van Witzenburg, W.; Mastenbroek, A. J. Nucl. Mater. 1985, 133-134, 553-557.

115. Singh, B. N.; Eldrup, M.; Zinkle, J. J.; Golubov, S. I. Phil. Mag. A 2002, 82, 1137-1158.

116. Samaras, M.; Derlet, P. M.; Van Swygenhoven, H.; Victoria, M. Phys. Rev. Lett. 88, 2002, 125505.

117. Rose, M.; Balogh, A. G.; Hahn, H. Nucl. Instr. Meth. Phys. Res. Sect. B 127-128, 1997, 119.

118. Chimi, Y.; Iwase, A.; Ishikawa, N.; Kobiyama, M.; Inami, T.; Okuda, S. J. Nucl. Mater. 2001, 297, 355.

119. Shen, T. D.; Feng, S.; Tang, M.; Valdez, J. A.; Wang, Y.; Sickafus, K. E. Appl. Phys. Lett. 90, 2007, 263115.

120. Samaras, M.; Derlet, P. M.; Van Swygenhoven, H. Phys. Rev. B 2003, 68, 224111.

121. Samaras, M.; Derlet, P. M.; Van Swygenhoven, H.; Victoria, M. Phil. Mag. 2003, 83, 3599-3607.

122. Stoller, R. E.; Kamenski, P. J.; Osetskiy, Yu. N. In Materials for Future Fusion and Fission Technologies; Fu, C. C., Kimura, A., Samaras, M., Serrano deCaro, M. Stoller, R. E., Eds.; Materials Research Society: Warrendale, PA, 2009; Vol. 1125, pp. 109-120. 
123. Voronoi, G. Z.; Reine, J. J. für die reine und angewandte Mathematik 2008, 134, 198-287.

124. Almazouzi, A.; Diaz de la Rubia, T.; Singh, B. N.; Victoria, M. J. Nucl. Mater. 2000, 276, 295-296.

125. Calder, A. F.; Bacon, D. J.; Barashev, A. V.; Osetsky, Yu. N. J. Nucl. Mater. 2008, 382, 91-95.

126. Becquart, C. S.; Domain, C.; van Duysen, J. C.; Raulot, J. M. J. Nucl. Mater. 2001, 394, 274-287.

127. Calder, A. F.; Bacon, D. J. In Symposium Proceedings on Microstructure Evolution During Irradiation; Robertson, I. M., Was, G. S., Hobbs, L. W., Diaz de la Rubia, T., Eds.; Materials Research Society: Pittsburgh, PA, 1997; Vol. 439, p. 521

128. Wallenius, J.; Abrikosov, I. A.; Chajarova, R.; et al. J. Nucl. Mater. 2004, 329-333, 1175-1179.

129. Malerba, L.; Caro, A.; Wallenius, J. J. Nucl. Mater. 2008, $382,112$.

130. Malerba, L.; Terentyev, D.; Olsson, P.; Chajarova, R.; Wallenius, J. J. Nucl. Mater. 2004, 329-333, 1156-1160.

131. Terentyev, D.; Malerba, L.; Hou, M. Nucl. Instr. Meth. B 2005, 228, 164-162.

132. Terentyev, D.; Malerba, L. J. Nucl. Mater. 2004, 329-333, $1161-1165$.

133. Klueh, R. L.; Harries, D. R. High-Chromium Ferritic and Martensitic Steels for Nuclear Applications, Monograph 3; American Society for Testing and Materials International: West Conshohocken, PA, 2001.

134. Ackland, G. J.; Mendelev, M. I.; Srolovitz, D. J.; Han, S.; Barashev, A. V. J. Phys. Cond. Mat. 2004, 16, S2629.

135. Okada, A.; Maeda, H.; Hamada, K.; Ishida, I. J. Nucl. Mater. 1999, 256, 247.
136. Vörtler, K.; Björkas, C.; Terentyev, D.; Malerba, L.; Nordlund, K. J. Nucl. Mater. 2008, 382, 24.

137. Björkas, C.; Nordlund, K.; Malerba, L.; Terentyev, D.; Olsson, P. J. Nucl. Mater. 2008, 372, 312.

138. Terentyev, D.; Malerba, L.; Chakarova, R.; et al. J. Nucl. Mater. 2006, 349, 119.

139. Voskoboinikov, R. E.; Osetsky, Yu. N.; Bacon, D. J. J. Nucl. Mater. 2008, 377, 385-395.

140. Voskoboinikov, R. E.; Osetsky, Yu. N.; Bacon, D. J. Nucl. Instr. Meth. Phys. Res. B 2006, 242, 68-70.

141. Voskoboinikov, R. E.; Osetsky, Yu. N.; Bacon, D. J. In Effects of Radiation on Materials; Allen, T. R., Lott, R. G., Busby, J. T., Kumar, A. S., Eds.; American Society for Testing and Materials International: West Conshohocken, PA, 2006; pp. 299-313, STP 1475 .

142. Bacorisen, D.; Smith, R.; Uberuaga, B. P.; Sickafus, K. E.; Ball, J. A.; Grimes, R. W. Nucl. Instr. Meth. B 2006, 250, 28-35.

143. Bishop, C. L.; Grimes, R. W.; Parfitt, D. C. Nucl. Instr. Meth. B 2010, 268, 2915-2917.

144. Aidhy, D. S.; Millett, P. C.; Desai, T.; Wolf, D.; Phillpot, S. R. Phys. Rev. B 2009, 80, 104107.

145. Dudarev, S. L.; Derlet, P. M. J. Phys. Cond. Mat. 2005, 17, 7097-7118.

146. Ackland, G. J. J. Nucl. Mater. 2006, 351, 20-27.

147. Malerba, L.; Ackland, G. J.; Becquart, C. S.; et al. J. Nucl. Mater. 2010, 406, 7-18.

148. Soneda, N.; Ishino, I.; Diaz de la Rubia, T. Phil. Mag. Lett. 2001, 81, 649-659. 